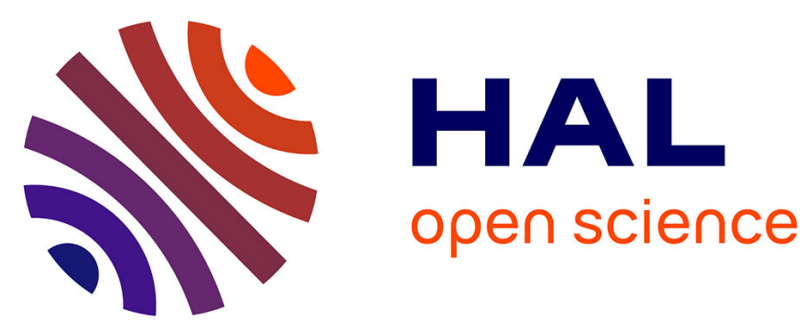

\title{
FEEDBACK STABILIZATION OF A TWO-DIMENSIONAL FLUID-STRUCTURE INTERACTION SYSTEM WITH MIXED BOUNDARY CONDITIONS
}

Jean-Pierre Raymond, Michel Fournié, Ndiaye Moctar

\section{To cite this version:}

Jean-Pierre Raymond, Michel Fournié, Ndiaye Moctar. FEEDBACK STABILIZATION OF A TWODIMENSIONAL FLUID-STRUCTURE INTERACTION SYSTEM WITH MIXED BOUNDARY

CONDITIONS. 2018. hal-01743783

\section{HAL Id: hal-01743783 \\ https://hal.science/hal-01743783}

Preprint submitted on 26 Mar 2018

HAL is a multi-disciplinary open access archive for the deposit and dissemination of scientific research documents, whether they are published or not. The documents may come from teaching and research institutions in France or abroad, or from public or private research centers.
L'archive ouverte pluridisciplinaire HAL, est destinée au dépôt et à la diffusion de documents scientifiques de niveau recherche, publiés ou non, émanant des établissements d'enseignement et de recherche français ou étrangers, des laboratoires publics ou privés. 


\title{
FEEDBACK STABILIZATION OF A TWO-DIMENSIONAL FLUID-STRUCTURE INTERACTION SYSTEM WITH MIXED BOUNDARY CONDITIONS
}

\author{
FOURNIÉ MICHEL*, NDIAYE MOCTAR*, AND RAYMOND JEAN-PIERRE*
}

\begin{abstract}
We study the stabilization of a fluid-structure interaction system around an unstable stationary solution. The system consists of coupling the incompressible Navier-Stokes equations, in a two dimensional polygonal domain with mixed boundary conditions, and a damped Euler-Bernoulli beam equations located at the boundary of the fluid domain. The control acts only in the beam equations. The feedback is determined by stabilizing the projection of the linearized model onto a finite dimensional invariant subspace. Here we have resolved two important challenges for applications in this field. One is the fact that we prove a stabilization result around a non zero stationary solution, which is new for such fluid-structure interaction systems. The other one is that the feedback laws that we determine do not depend on the Leray projector used to get rid of the algebraic constraints of partial differential equations. This is essential for numerical aspects.
\end{abstract}

Key words. Fluid-structure interaction, feedback control, stabilization, Navier-Stokes equations, beam equation.

AMS subject classifications. 93B52, 93C20, 93D15, 76D55, 76D05, 74F10.

1. Introduction. We are interested in stabilizing, around a non-zero stationary solution, a fluidstructure interaction system coupling beam equations and the Navier-Stokes equations. The initial configuration for the fluid domain is denoted by $\Omega$, and is refered as the reference configuration. Its boundary $\Gamma$ is split into different parts $\Gamma=\Gamma_{s} \cup \Gamma_{i} \cup \Gamma_{e} \cup \Gamma_{n}$, where $\Gamma_{s}$ is a flat part of the boundary occupied by elastic structures satisfying Euler-Bernoulli damped beam equations. For the fluid, Neumann boundary conditions are prescribed on $\Gamma_{n}$, Navier type boundary conditions are prescribed on $\Gamma_{e}$, and Dirichlet boundary conditions are prescribed on $\Gamma_{i}$. The assumptions that are essential in our analysis are listed in Section 2. A particular configuration satisfying these assumptions corresponds to the right hand side of a wind tunnel, see Figure 1.1, in which a fluid flows around a thick plate, and two beams are located in the upper and lower boundaries of the plate. In Figure 1.1, the domain $\Omega$ is polygonal but the results of the paper can be easily extended to other geometrical configurations, see Remark 2.1. The stabilization problem for the associated semi-discrete model is studied in [8].

We assume that the inflow boundary condition in the computational domain $\Omega$ is a perturbed Blasius type profile, which is used to determine the stationary solution around which we want to stabilize the fluid-structure system. The goal is to use a force term as control in the beam equations in order to stabilize the full fluid-structure system. Since the structure is deformed under the action of the fluid, the domain occupied by the fluid at time $t$ depends on the displacement $\eta(t)$ of the structure, see Figure 1.2. The fluid domain at time $t$ is denoted by $\Omega_{\eta(t)}$ and the fluid-structure interface by $\Gamma_{\eta(t)}$. We use the notations

$$
\begin{aligned}
& Q_{\eta}^{\infty}=\bigcup_{t \in(0, \infty)}\left(\{t\} \times \Omega_{\eta(t)}\right), \quad \Sigma_{\eta}^{\infty}=\bigcup_{t \in(0, \infty)}\left(\{t\} \times \Gamma_{\eta(t)}\right), \quad Q^{\infty}=(0, \infty) \times \Omega, \\
& \Sigma_{s}^{\infty}=(0, \infty) \times \Gamma_{s}, \quad \Sigma_{i}^{\infty}=(0, \infty) \times \Gamma_{i}, \quad \Sigma_{e}^{\infty}=(0, \infty) \times \Gamma_{e}, \quad \Sigma_{n}^{\infty}=(0, \infty) \times \Gamma_{n} .
\end{aligned}
$$

The Eulerian-Lagrangian system describing the evolution of the fluid-structure system is

$$
\begin{aligned}
& u_{t}-\operatorname{div} \sigma(u, p)+(u \cdot \nabla) u=0, \quad \operatorname{div} u=0 \text { in } Q_{\eta}^{\infty}, \\
& u=\eta_{t} n \text { on } \Sigma_{\eta}^{\infty}, \quad u=g_{s}+g_{p} \text { on } \Sigma_{i}^{\infty}, \quad u \cdot n=0 \text { and } \varepsilon(u) n \cdot \tau=0 \text { on } \Sigma_{e}^{\infty}, \\
& \sigma(u, p) n=0 \text { on } \Sigma_{n}^{\infty}, \quad u(0)=u^{0} \text { on } \Omega, \\
& \eta_{t t}-\beta \Delta_{s} \eta-\gamma \Delta_{s} \eta_{t}+\alpha \Delta_{s}^{2} \eta=-\left.\sigma(u, p)\right|_{\Gamma_{\eta(t)}} n_{\eta(t)} \sqrt{1+\eta_{x}^{2}} \cdot n+f_{s}+f \text { on } \Sigma_{s}^{\infty}, \\
& \eta=0 \text { on }(0, \infty) \times \partial \Gamma_{s}, \quad \eta_{x}=0 \text { on }(0, \infty) \times \partial \Gamma_{s}, \\
& \eta(0)=0 \text { on } \Gamma_{s}, \quad \eta_{t}(0)=\eta_{2}^{0} \text { on } \Gamma_{s},
\end{aligned}
$$

where $u$ and $p$ stand for the fluid velocity and pressure, $\sigma(u, p)$ is the Cauchy stress tensor

$$
\sigma(u, p)=2 \nu \varepsilon(u)-p I, \quad \varepsilon(u)=\frac{1}{2}\left(\nabla u+(\nabla u)^{T}\right),
$$

*Institut de Mathématiques de Toulouse, UMR 5219, Université Paul Sabatier Toulouse III \& CNRS, 31062 Toulouse Cedex, France (michel.fournie@math.univ-toulouse.fr, moctar.ndiaye@math.univ-toulouse.fr, jeanpierre.raymond@math.univ-toulouse.fr) 


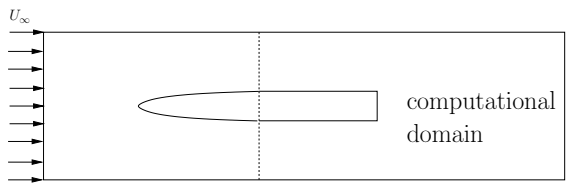

Fig. 1.1: Configuration of the wind tunnel.

$\nu$ is the fluid viscosity, $\alpha>0, \beta \geq 0$ and $\gamma>0$ are parameters of the structure, $f_{s}$ is a stationary force precisely defined below after $(1.2)$, and $n_{\eta(t)}($ resp. $n)$ is the unit normal to $\Gamma_{\eta(t)}$ (resp. $\Gamma_{s}$ ) exterior to $\Omega_{\eta(t)}$ (resp. $\Omega$ ). Here $\Delta_{s}=\partial_{x x}$ stands for the Laplace-Beltrami operator on $\Gamma_{s}$. Clamped boundary conditions are imposed at the extremities of the beams, and $\eta$ is a vector valued function representing the displacements of the upper and lower beams. The inflow boundary condition $g_{s} \in H^{2}\left(\Gamma_{i} ; \mathbb{R}^{2}\right)$ is independent of time and $g_{p}$ is a time dependent perturbation of $g_{s}$, taking into account the fact that the inflow boundary condition of the computational domain $\Omega$ is not precisely known. The precise assumptions on $g_{s}$ are stated in Section 2.2. The function $f_{s}$ is also assumed to be time independent, and the control function $f$ is taken in the form

$$
f(t, x, y)=\sum_{i=1}^{n_{c}} f_{i}(t) w_{i}(x, y),
$$

where the functions $w_{i} \in L^{2}\left(\Gamma_{s}\right)$ are chosen in (8.4), so that some stabilizability condition stated in (8.7) is satisfied.

Let $\left(u_{s}, p_{s}\right)$ be a solution of the stationary Navier-Stokes equations

$$
\begin{aligned}
& -\operatorname{div} \sigma\left(u_{s}, p_{s}\right)+\left(u_{s} \cdot \nabla\right) u_{s}=0, \quad \operatorname{div} u_{s}=0 \text { in } \Omega, \\
& u_{s}=0 \text { on } \Gamma_{s}, \quad u_{s}=g_{s} \text { on } \Gamma_{i}, \quad u_{s} \cdot n=0 \text { and } \varepsilon\left(u_{s}\right) n \cdot \tau=0 \text { on } \Gamma_{e}, \quad \sigma\left(u_{s}, p_{s}\right) n=0 \text { on } \Gamma_{n} .
\end{aligned}
$$

We choose $f_{s}=-\left.p_{s}\right|_{\Gamma_{s}}$ in $(1.1)_{5}$. Thus, $(u, p, \eta)=\left(u_{s}, p_{s}, 0\right)$ is a stationary solution of system (1.1). We assume that it is an unstable stationary solution of that system. The goal of the paper is to find a control variable $\mathbf{f}=\left(f_{1}, \cdots, f_{n_{c}}\right)$, in feedback form, able to stabilize system (1.1) around the stationary solution $\left(u_{s}, p_{s}, 0\right)$, with any prescribed exponential decay rate $-\omega<0$, provided that $g_{p}, u^{0}-u_{s}$ and $\eta_{2}^{0}$ are small enough in appropriate functional spaces.

We follow a classical approach consisting of finding a feedback control law, stabilizing a linearized model, that is next applied to the nonlinear system. Let us explain why implementing this approach is not obvious and leads to new difficulties. In (1.1) the main nonlinearities come from the fact that the Navier-Stokes system is written in a time-dependent geometrical domain depending on the displacement of elastic structures. Therefore, before linearizing the system, we have to rewrite it in the reference configuration $(0, \infty) \times \Omega$ (see Section 3). For the stability analysis of our linearized fluid-structure system and for stabilization issues, we have to study the direct and adjoint eigenvalue problems. These problems are not standard because the algebraic constraints of the direct eigenvalue problem are

$$
\operatorname{div} v=A_{3} \eta_{1} \text { in } \Omega, \quad v=\eta_{2} n \text { on } \Gamma_{s},
$$

(see system (5.1)), while the algebraic constraints of the adjoint eigenvalue problem are

$$
\operatorname{div} \phi=0 \text { in } \Omega, \quad \phi=\xi_{2} n \text { on } \Gamma_{s},
$$

(see system (6.2)). It is a consequence of the fact that the system is linearized around a non zero stationary solution. Because the algebraic constraints are different, studying the spectrum and performing a Jordan decomposition of the linearized operator is not standard. In order to study the spectrum of the linearized operator and to establish its link with the direct and adjoint eigenvalue problems in PDE formulation, we have to rewrite them into equivalent forms with eigenfunctions belonging to the same state space. This can be done by using the so-called Leray projector to transform the direct and adjoint eigenvalue problems. This approach leads to direct and adjoint eigenvalue problems in the form of operator equations. Let 
us emphasize that operator formulation is needed to justify the eigenvalue analysis, and that the PDE formulation will be needed in [8] for numerical simulations. For that, we have to establish the equivalence between these two formulations. This is done in Section 7 .

To the best of our knowledge, the results of the present paper are the first ones dealing with the stabilization of a fluid-structure system involving mixed boundary conditions in the fluid equations. The analysis of such a system seems also to be new in the literature.

Feedback stabilization of strong solutions of such a system around the null solution, by controls acting only in the equations of the structure, is treated in [24]. In [1], the authors obtained stabilization results by means of Dirichlet boundary controls acting in the fluid equations. Several existence results of strong and weak solutions, and some uniqueness results, have been proved in $[6,10,2,16,11]$.

The plan of the paper is as follows. The functional setting and the assumptions are given in Section 2. The main result is stated in Section 3. The analysis of Oseen operator and the characterization of the pressure in the Oseen system are done in Section 4. In Section 5, we characterize the infinitesimal generator $(\mathcal{A}, D(\mathcal{A}))$ of the semigroup corresponding to the linearized system. We prove that this semigroup is analytic with compact resolvent. The adjoint of $(\mathcal{A}, D(\mathcal{A}))$ is characterized in Section 6 . The equivalences between eigenvalue problems are established in Section 7. In Section 8, we prove that the bi-orthogonality condition satisfied by the families of eigenfunctions of the direct and adjoint partial differential equations implies another bi-orthogonality condition satisfied by the families of eigenfunctions of the direct and adjoint operator equations. This new relationship is used to define a feedback control law independent of the Leray projector, and which can be easily calculated (see [8]).
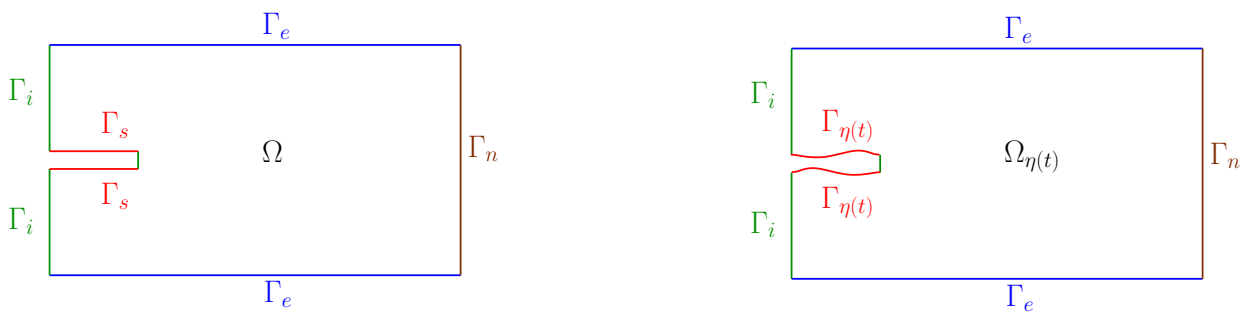

Fig. 1.2: Reference configuration (left) and deformed configuration (right).

\section{Functional setting.}

2.1. Notations. The geometrical domain $\Omega$ and $\Gamma_{s}$, see Figure 1.2, are defined by

$$
\Omega=([0, L] \times[-\ell, \ell]) \backslash\left(\left[0, \ell_{s}\right] \times[-e, e]\right), \quad \Gamma_{s}=\left(0, \ell_{s}\right) \times\{e\} \cup\left(0, \ell_{s}\right) \times\{-e\},
$$

where $L>0$ is the length of the computational domain, $2 \ell$ is its height, $\ell_{s}$ is the length of the thick plate, and $2 e$ is the thickness of the plate. Thus equations $(1.1)_{5-7}$ describe the displacement of the upper beam located at $\left(0, \ell_{s}\right) \times\{e\}$ and the lower beam located at $\left(0, \ell_{s}\right) \times\{-e\}$. We also have

$$
\begin{aligned}
& \Gamma_{i}=\Gamma_{i, 1} \cup \Gamma_{i, 2} \cup \Gamma_{i, 3} \quad \text { with } \quad \Gamma_{i, 1}=\{0\} \times(-\ell,-e), \quad \Gamma_{i, 2}=\{0\} \times(e, \ell), \quad \Gamma_{i, 3}=\left\{\ell_{s}\right\} \times(-e, e), \\
& \Gamma_{e}=((0, L) \times\{-\ell\}) \cup((0, L) \times\{\ell\}), \quad \Gamma_{i, e}=\Gamma_{i} \cup \Gamma_{e}, \quad \Gamma_{n}=\{L\} \times(-\ell, \ell), \quad \Gamma_{0}=\Gamma_{s} \cup \Gamma_{i, e} .
\end{aligned}
$$

We introduce the functional spaces

$$
\begin{aligned}
& \mathbf{L}^{2}(\Omega)=L^{2}\left(\Omega ; \mathbb{R}^{2}\right), \quad \mathbf{H}^{s}(\Omega)=H^{s}\left(\Omega ; \mathbb{R}^{2}\right), \quad \forall s>0, \\
& \mathbf{H}_{\Gamma_{0}}^{s}(\Omega)=\left\{v \in \mathbf{H}^{s}(\Omega) \mid v=0 \text { on } \Gamma_{s} \cup \Gamma_{i}, v \cdot n=0 \text { on } \Gamma_{e}\right\}, \quad \forall s>\frac{1}{2}, \\
& \mathbf{V}_{n, \Gamma_{0}}^{0}(\Omega)=\left\{v \in \mathbf{L}^{2}(\Omega) \mid \operatorname{div} v=0 \text { on } \Omega, v \cdot n=0 \text { on } \Gamma_{0}\right\}, \\
& \mathbf{V}_{n, \Gamma_{0}}^{s}(\Omega)=\mathbf{H}^{s}(\Omega) \cap \mathbf{V}_{n, \Gamma_{0}}^{0}(\Omega), \quad \forall s>0, \\
& \mathbf{V}_{\Gamma_{i, e}}^{s}(\Omega)=\left\{v \in \mathbf{H}^{s}(\Omega) \mid \operatorname{div} v=0 \text { in } \Omega, v=0 \text { on } \Gamma_{i}, v \cdot n=0 \text { on } \Gamma_{e}\right\}, \quad \forall s>\frac{1}{2} .
\end{aligned}
$$

We also introduce the product spaces

$$
H=\mathbf{L}^{2}(\Omega) \times H_{0}^{2}\left(\Gamma_{s}\right) \times L^{2}\left(\Gamma_{s}\right) \quad \text { and } \quad Z=\mathbf{V}_{n, \Gamma_{0}}^{0}(\Omega) \times H_{0}^{2}\left(\Gamma_{s}\right) \times L^{2}\left(\Gamma_{s}\right) .
$$


The space $\mathbf{V}_{n, \Gamma_{0}}^{0}(\Omega)$ is equipped with the $\mathbf{L}^{2}$-norm, the spaces $\mathbf{H}_{\Gamma_{0}}^{s}(\Omega), \mathbf{V}_{\Gamma_{i, e}}^{s}(\Omega)$ and $\mathbf{V}_{n, \Gamma_{0}}^{s}(\Omega)$ are equipped with the $\mathbf{H}^{s}$-norm. The inner product in $H_{0}^{2}\left(\Gamma_{s}\right)$ is chosen at the beginning of Section 5.2, and the inner product in $H$ and $Z$ is defined in (5.2).

The space of boundary displacements. We consider displacements of the beams located on $\Gamma_{s}$ belonging to the space denoted by $H_{0}^{\frac{3}{2}}\left(\Gamma_{s}\right)$, which is identified with $\left(H_{0}^{\frac{3}{2}}\left(0, \ell_{s}\right)\right)^{2}=\left(H^{\frac{3}{2}}\left(0, \ell_{s}\right) \cap H_{0}^{1}\left(0, \ell_{s}\right)\right)^{2}$. By writing $H_{0}^{\frac{3}{2}}\left(\Gamma_{s}\right)$, we emphasize the fact that $\eta$ is a vector function with one component defined on $\left(0, \ell_{s}\right) \times\{e\}$ and the other one defined on $\left(0, \ell_{s}\right) \times\{-e\}$.

The space of inflow boundary conditions. On the boundary $\Gamma_{i}=\Gamma_{i, 1} \cup \Gamma_{i, 2} \cup \Gamma_{i, 3}$, we consider inflow conditions $\mathbf{g}$ belonging to the space

$$
\begin{gathered}
\mathbf{H}\left(\Gamma_{i}\right)=\left\{\mathbf{g}=\left(g_{1}, g_{2}\right)\left|g_{2}=0, g_{1}\right|_{\Gamma_{i, 3}}=0,\left.g_{1}\right|_{\Gamma_{i, 1}} \in H^{2}(-\ell,-e),\left.g_{1}\right|_{\Gamma_{i, 2}} \in H^{2}(e, \ell),\right. \\
\left.g_{1}^{\prime}(-\ell)=g_{1}^{\prime}(\ell)=0, g_{1}(-e)=g_{1}(e)=0\right\} .
\end{gathered}
$$

The space $\mathbf{H}\left(\Gamma_{i}\right)$ is equipped with the norm $\left(g_{1}, g_{2}\right) \longmapsto\left\|g_{1}\right\|_{H^{2}((-\ell,-e) \cup(e, \ell))}$.

We denote by $\mathcal{C}=\left(C_{j}\right)_{1 \leq j \leq 8}$ the set of the corners of $\Omega$. For all $-1<\delta<1, s \in \mathbb{N}$, and for $n=1$ or $n=2$, we introduce the norms

$$
\|v\|_{H_{\delta}^{s}\left(\Omega ; \mathbb{R}^{n}\right)}^{2}=\sum_{|\alpha| \leq s} \int_{\Omega} r^{2 \delta}\left|\partial_{\alpha} v\right|^{2}
$$

where $r$ stands for the distance to $\mathcal{C}, \alpha=\left(\alpha_{1}, \alpha_{2}\right) \in \mathbb{N}^{2}$ denotes a two-index, $|\alpha|=\alpha_{1}+\alpha_{2}$ is its length, $\partial_{\alpha}$ denotes the corresponding partial differential operator. We denote by $H_{\delta}^{s}\left(\Omega ; \mathbb{R}^{n}\right)$ the closure of $C^{\infty}(\bar{\Omega})$ in the norm $\|\cdot\|_{H_{\delta}^{s}\left(\Omega ; \mathbb{R}^{n}\right)}$. We set

$$
\begin{aligned}
& \mathbf{L}_{\delta}^{2}(\Omega)=H_{\delta}^{0}\left(\Omega ; \mathbb{R}^{2}\right), \quad \mathbf{H}_{\delta}^{s}(\Omega)=H_{\delta}^{s}\left(\Omega ; \mathbb{R}^{2}\right) \text { for all } s>0, \\
& H_{\delta}=\mathbf{H}_{\delta}^{2}(\Omega) \times H_{\delta}^{1}(\Omega) \times\left(H^{4}\left(\Gamma_{s}\right) \cap H_{0}^{2}\left(\Gamma_{s}\right)\right) \times H_{0}^{2}\left(\Gamma_{s}\right) .
\end{aligned}
$$

We also introduce the following spaces of time dependent functions

$$
\begin{aligned}
& H^{2,1}\left(Q^{\infty}\right)=L^{2}\left(0, \infty ; \mathbf{H}^{2}(\Omega)\right) \cap H^{1}\left(0, \infty ; \mathbf{L}^{2}(\Omega)\right), \quad H_{\delta}^{2,1}\left(Q^{\infty}\right)=L^{2}\left(0, \infty ; \mathbf{H}_{\delta}^{2}(\Omega)\right) \cap H^{1}\left(0, \infty ; \mathbf{L}^{2}(\Omega)\right), \\
& H_{0}^{2,1}\left(\Sigma_{s}^{\infty}\right)=L^{2}\left(0, \infty ; H_{0}^{2}\left(\Gamma_{s}\right)\right) \cap H^{1}\left(0, \infty ; L^{2}\left(\Gamma_{s}\right)\right), \quad H_{0}^{4,2}\left(\Sigma_{s}^{\infty}\right)=H_{0}^{2,1}\left(\Sigma_{s}^{\infty}\right) \cap H^{4,2}\left(\Sigma_{s}^{\infty}\right), \\
& Y=L^{2}\left(0, \infty ; \mathbf{L}^{2}(\Omega)\right) \times H^{2,1}\left(Q^{\infty}\right) \times L^{2}\left(0, \infty ; L^{2}\left(\Gamma_{s}\right)\right), \\
& X_{\delta}=H_{\delta}^{2,1}\left(Q^{\infty}\right) \times L^{2}\left(0, \infty ; H_{\delta}^{1}(\Omega)\right) \times H_{0}^{4,2}\left(\Sigma_{s}^{\infty}\right) \times H_{0}^{2,1}\left(\Sigma_{s}^{\infty}\right) .
\end{aligned}
$$

\subsection{Assumptions.}

Assumption 1. We assume that $g_{s} \in \mathbf{H}\left(\Gamma_{i}\right)$, and that the system (1.2) admits a solution $\left(u_{s}, p_{s}\right) \in$ $\mathbf{H}_{\delta_{0}}^{2}(\Omega) \times H_{\delta_{0}}^{1}(\Omega)$ for some $0<\delta_{0}<\frac{1}{2}$ specified in $(4.4)$.

Assumptions 2 AND 3. These assumptions are stated in Section 8.2, see (8.5) and (8.7).

In Assumption 2, we state that the parts of spectrum of the Oseen operator and that of the elastic structure contained in the half plane $\{\lambda \in \mathbb{C} \mid \operatorname{Re} \lambda \geq-\omega\}$ are disjoint. In Assumption 3, we state a unique continuation property sufficient to verify the Hautus stabilization test for the linearized system.

REMARK 2.1. The main results of the paper, Theorems 3.2 and 3.3, are proved for the geometrical configuration described in Section 2.1 and under Assumptions 1, 2 and 3. As mentioned in the introduction, Theorems 3.2 and 3.3 remain valid for geometrical configurations for which the regularity result stated in Theorem 4.1 is true. (See e.g. [21] where other geometrical configuration are considered.)

3. System in the reference configuration and main results. We denote by $\mathcal{E}$ a continuous linear operator from $H_{0}^{4,2}\left(\Sigma_{s}^{\infty}\right)$ into $H_{0}^{4,2}((0, \infty) \times(0, L) \times\{e,-e\})$ such that $\mathcal{E} \eta(t, x)=0$ for $(t, x) \in(0, \infty) \times\left(\left(\ell_{s}+L\right) / 2, L\right)$. For beam displacements $\eta \in H_{0}^{4,2}\left(\Sigma_{s}^{\infty}\right)$ small enough, we define a $\mathcal{C}^{1}$-diffeomorphism $\mathcal{T}_{\eta(t)}$, from $\Omega_{\eta(t)}$ into $\Omega$, by setting

$$
\mathcal{T}_{\eta(t)}(x, y)=(x, z)=\left(x, \frac{(\ell-e) y-\ell \mathcal{E} \eta(t, x, s(y) e)}{\ell-e-s(y) \mathcal{E} \eta(t, x, s(y) e)}\right),
$$


where the sign function $s$ is defined by $\quad s(y)=-1$ if $y<0 \quad$ and $\quad s(y)=1$ if $y \geq 0$.

REMARK 3.1. To simplify the notation, in all the transformations, the nonlinear terms and the operators involving $\mathcal{E} \eta$ for some $\eta \in H_{0}^{4,2}\left(\Sigma_{s}^{\infty}\right)$, we shall simply write $\eta$ in place of $\mathcal{E} \eta$. We shall do the same abuse of notation when the partial derivatives of $\mathcal{E} \eta$ appear in some expression.

For a given $\eta \in H_{0}^{4,2}\left(\Sigma_{s}^{\infty}\right)$ such that the mapping $\mathcal{T}_{\eta(t)}$ is a $\mathcal{C}^{1}$-diffeomorphism from $\Omega_{\eta(t)}$ into $\Omega$ for all $t>0$, we say that $u$ belongs to $H_{\delta}^{2,1}\left(Q_{\eta}^{\infty}\right)$ (resp. $p$ belongs to $L^{2}\left(0, \infty ; H_{\delta}^{1}\left(\Omega_{\eta}\right)\right)$ ) if and only if

$$
u\left(\cdot, \mathcal{T}_{\eta(\cdot)}^{-1}\right) \text { belongs to } H_{\delta}^{2,1}\left(Q^{\infty}\right)\left(\text { resp. } p\left(\cdot, \mathcal{T}_{\eta(\cdot)}^{-1}\right) \text { belongs to } L^{2}\left(0, \infty ; H_{\delta}^{1}(\Omega)\right)\right) \text {. }
$$

The main result of the paper is the following theorem.

TheOREm 3.2. Let $\omega$ be positive. We assume that Assumptions 1, 2 and 3 are satisfied. There exists $r>0$ such that, for all $u^{0} \in \mathbf{H}^{1}(\Omega), \eta_{2}^{0} \in H_{0}^{1}\left(\Gamma_{s}\right)$, and $e^{\omega t} g_{p} \in H_{0}^{1}\left(0, \infty ; \mathbf{H}\left(\Gamma_{i}\right)\right)$ satisfying $u^{0}-u_{s} \in \mathbf{V}_{\Gamma_{i, e}}^{1}(\Omega),\left.\left(u^{0}-u_{s}\right)\right|_{\Gamma_{s}}=\eta_{2}^{0} n$ and

$$
\left\|u^{0}-u_{s}\right\|_{\mathbf{H}^{1}(\Omega)}+\left\|\eta_{2}^{0}\right\|_{H_{0}^{1}\left(\Gamma_{s}\right)}+\left\|e^{\omega t} g_{p}\right\|_{H_{0}^{1}\left(0, \infty ; \mathbf{H}\left(\Gamma_{i}\right)\right)} \leq r,
$$

we can find $\mathbf{f}=\left(f_{1}, \cdots, f_{n_{c}}\right) \in H^{1}\left(0, \infty ; \mathbb{R}^{n_{c}}\right)$, in feedback form, for which the nonlinear system (1.1) admits a solution $(u, p, \eta)$ in $H_{\delta_{0}}^{2,1}\left(Q_{\eta}^{\infty}\right) \times L^{2}\left(0, \infty ; H_{\delta_{0}}^{1}\left(\Omega_{\eta(t)}\right)\right) \times H^{4,2}\left(\Sigma_{s}^{\infty}\right)$, for $\delta_{0}$ defined in $(4.4)$, satisfying

$$
\begin{aligned}
& \left\|\left(u\left(t, \mathcal{T}_{\eta(t)}^{-1}\right)-u_{s}, \eta(t), \eta_{t}(t)\right)\right\|_{\mathbf{H}^{\frac{3}{4}+\frac{\varepsilon_{0}}{2}}(\Omega) \times H^{3}\left(\Gamma_{s}\right) \times H^{1}\left(\Gamma_{s}\right)} \leq C e^{-\omega t}, \quad \forall t>0, \quad \text { and } \\
& \left\|e^{-\omega \cdot} \eta_{1}\right\|_{L^{\infty}\left(\Sigma_{s}^{\infty}\right)}<e,
\end{aligned}
$$

where $C>0$ depends on $r$ and $0<\varepsilon_{0}<\frac{1}{2}$.

In order the prove that theorem, we make the change of unknowns

$$
\begin{aligned}
& \widehat{u}(t, x, z):=e^{\omega t}\left[u\left(t, \mathcal{T}_{\eta(t)}^{-1}(x, z)\right)-u_{s}(x, z)\right], \quad \widehat{p}(t, x, z):=e^{\omega t}\left[p\left(t, \mathcal{T}_{\eta(t)}^{-1}(x, z)\right)-p_{s}(x, z)\right], \\
& \widehat{\eta}_{1}(t, x):=e^{\omega t} \eta(t, x), \quad \widehat{\eta}_{2}(t, x):=e^{\omega t} \eta_{t}(t, x), \quad \widehat{\mathbf{f}}(t)=\left(\widehat{f}_{i}(t)\right)_{1 \leq i \leq n_{c}}:=e^{\omega t} \mathbf{f}(t), \\
& \widehat{g}_{p}(t, x, z):=e^{\omega t} g_{p}(t, x, z) \quad \text { and } \quad \widehat{u}^{0}=u^{0}-u_{s} .
\end{aligned}
$$

The quadruplet $\left(\widehat{u}, \widehat{p}, \widehat{\eta}_{1}, \widehat{\eta}_{2}\right)$ satisfies the system

$$
\begin{aligned}
& \widehat{u}_{t}-\operatorname{div} \sigma(\widehat{u}, \widehat{p})+\left(u_{s} \cdot \nabla\right) \widehat{u}+(\widehat{u} \cdot \nabla) u_{s}-A_{1} \widehat{\eta}_{1}-A_{2} \widehat{\eta}_{2}-\omega \widehat{u}=e^{-\omega t} \mathcal{F}_{f}\left[\widehat{u}, \widehat{p}, \widehat{\eta}_{1}, \widehat{\eta}_{2}\right] \text { in } Q^{\infty}, \\
& \operatorname{div} \widehat{u}=A_{3} \widehat{\eta}_{1}+e^{-\omega t} \operatorname{div} \mathcal{F}_{\operatorname{div}}\left[\widehat{u}, \widehat{\eta}_{1}\right] \text { in } Q^{\infty}, \quad \widehat{u}=\widehat{\eta}_{2} n \text { on } \Sigma_{s}^{\infty}, \quad \widehat{u}=\widehat{g}_{p} \text { on } \Sigma_{i}^{\infty}, \\
& \widehat{u} \cdot n=0 \text { on } \Sigma_{e}^{\infty}, \quad \varepsilon(\widehat{u}) n \cdot \tau=0 \text { on } \Sigma_{e}^{\infty}, \quad \sigma(\widehat{u}, \widehat{p}) n=0 \text { on } \Sigma_{n}^{\infty}, \\
& \widehat{\eta}_{1, t}-\widehat{\eta}_{2}-\omega \widehat{\eta}_{1}=0 \text { on } \Sigma_{s}^{\infty} \\
& \widehat{\eta}_{2, t}-\beta \Delta_{s} \widehat{\eta}_{1}-\gamma \Delta_{s} \widehat{\eta}_{2}+\alpha \Delta_{s}^{2} \widehat{\eta}_{1}-A_{4} \widehat{\eta}_{1}-\omega \widehat{\eta}_{2}=\gamma_{s} \widehat{p}+e^{-\omega t} \mathcal{F}_{s}\left[\widehat{u}, \widehat{\eta}_{1}\right]+\widehat{f} \text { on } \Sigma_{s}^{\infty}, \\
& \widehat{\eta}_{1}=0 \text { on }(0, \infty) \times \partial \Gamma_{s}, \quad \widehat{\eta}_{1, x}=0 \text { on }(0, \infty) \times \partial \Gamma_{s}, \\
& \widehat{u}(0)=\widehat{u}^{0} \text { in } \Omega, \quad \widehat{\eta}_{1}(0)=0 \text { on } \Gamma_{s}, \quad \widehat{\eta}_{2}(0)=\eta_{2}^{0} \text { on } \Gamma_{s},
\end{aligned}
$$

where $\gamma_{s}$ is the trace operator on $\Gamma_{s}$, the nonlinear terms $\mathcal{F}_{f}$ and $\mathcal{F}_{s}$ are given in Appendix A, $\mathcal{F}_{\text {div }}\left[\widehat{u}, \widehat{\eta}_{1}\right]$, and the linear differential operators $A_{1}, A_{2}, A_{3}$ and $A_{4}$ are defined by

$$
\begin{aligned}
\mathcal{F}_{\text {div }}\left[\widehat{u}, \widehat{\eta}_{1}\right]= & \frac{1}{\ell-e}\left(s(z) \widehat{\eta}_{1} \widehat{u}_{1} e_{1}+(\ell-s(z) z) \widehat{\eta}_{1, x} \widehat{u}_{1} e_{2}\right), \quad e_{1}=(1,0)^{T}, \quad e_{2}=(0,1)^{T}, \\
A_{1} \widehat{\eta}_{1}= & \frac{s(z) \widehat{\eta}_{1}}{\ell-e}\left(p_{s, z} e_{2}+u_{s, 2} u_{s, z}-2 \nu u_{s, z z}+\nu u_{s, 1, x x} e_{1}+\nu u_{s, 1, x z} e_{2}\right) \\
& +\frac{(\ell-s(z) z) \widehat{\eta}_{1, x}}{\ell-e}\left(p_{s, z} e_{1}-2 \nu u_{s, x z}+u_{s, 1} u_{s, z}-\nu u_{s, 1, z z} e_{2}-\nu u_{s, 1, x z} e_{1}\right) \\
& +\frac{\nu \widehat{\eta}_{1, x}}{\ell-e}\left(u_{s, 1, z} e_{2}-u_{s, 1, x} e_{1}\right)-\frac{\nu(\ell-s(z) z) \widehat{\eta}_{1, x x}}{\ell-e}\left(u_{s, z}+u_{s, 1, z} e_{1}\right), \\
A_{2} \widehat{\eta}_{2}= & \frac{(\ell-s(z) z) \widehat{\eta}_{2}}{\ell-e} u_{s, z}, \quad A_{3} \widehat{\eta}_{1}=\frac{1}{\ell-e}\left(s(z) \widehat{\eta}_{1} u_{s, 1, x}+(\ell-s(z) z) \widehat{\eta}_{1, x} u_{s, 1, z}\right), \\
A_{4} \widehat{\eta}_{1}= & \nu\left(\frac{s(z) \widehat{\eta}_{1}}{\ell-e} u_{s, 2, z}-\widehat{\eta}_{1, x} u_{s, 1, z}-2 \gamma_{s}\left(A_{3} \widehat{\eta}_{1}\right)\right) .
\end{aligned}
$$


We notice that we have used the condition $\operatorname{div} \widehat{u}=A_{3} \widehat{\eta}_{1}+e^{-\omega t} \operatorname{div} \mathcal{F}_{\operatorname{div}}\left[\widehat{u}, \widehat{\eta}_{1}\right]$, and the boundary condition $\left.\widehat{u}_{1}\right|_{\Sigma_{s}^{\infty}}=0$ to replace $2 \nu \widehat{u}_{2, z}$, which appears in equation $(3.3)_{5}$, by

$$
2 \nu \gamma_{s}\left(A_{3} \widehat{\eta}_{1}+e^{-\omega t} \operatorname{div} \mathcal{F}_{\operatorname{div}}\left[\widehat{u}, \widehat{\eta}_{1}\right]\right) .
$$

The term $2 \nu \gamma_{s}\left(A_{3} \widehat{\eta}_{1}\right)$ appears in $A_{4} \widehat{\eta}_{1}$, while the term $2 \nu \gamma_{s}\left(\operatorname{div} \mathcal{F}_{\text {div }}\left[\widehat{u}, \widehat{\eta}_{1}\right]\right)$ is involved in the nonlinear term $\mathcal{F}_{s}$ of the equation.

In the nonlinear terms $\mathcal{F}_{f}, \mathcal{F}_{s}$ and $\mathcal{F}_{\text {div }}$, the functions $\widehat{\eta}_{1}$ and $\widehat{\eta}_{2}$, which are a priori defined over $\left[0, \ell_{s}\right]$, are extended by using the operator $\mathcal{E}$, see Remark 3.1 . We can easily check that, if $\widehat{u} \cdot n=0$ and $\varepsilon(\widehat{u}) n \cdot \tau=0$ on $\Sigma_{e}^{\infty}$, then $\mathcal{F}_{\text {div }}=\mathcal{F}_{\text {div }}\left[\widehat{u}, \widehat{\eta}_{1}\right]$ satisfies the following boundary conditions

$$
\begin{aligned}
& \mathcal{F}_{\text {div }}=0 \text { on } \Sigma_{s}^{\infty}, \quad \mathcal{F}_{\text {div }}=0 \text { on } \Sigma_{i}^{\infty}, \quad \mathcal{F}_{\text {div }} \cdot n=0 \text { on } \Sigma_{e}^{\infty}, \\
& \varepsilon\left(\mathcal{F}_{\text {div }}\right) n \cdot \tau=0 \text { on } \Sigma_{e}^{\infty}, \quad \varepsilon\left(\mathcal{F}_{\text {div }}\right) n=0 \text { on } \Sigma_{n}^{\infty} .
\end{aligned}
$$

Moreover $\left.\mathcal{F}_{\text {div }}\right|_{t=0}=0$ since $\left.\widehat{\eta}_{1}\right|_{t=0}=0$. Theorem 3.2 is a direct consequence of the following result.

THEOREM 3.3. Let $\eta_{\max }$ be in $(0, e)$ and $\omega$ be positive. We assume that Assumptions 1, 2 and 3 are satisfied. There exists $r>0$ such that, for all $\widehat{u}^{0} \in \mathbf{V}_{\Gamma_{i, e}}^{1}(\Omega), \eta_{2}^{0} \in H_{0}^{1}\left(\Gamma_{s}\right)$, and $\widehat{g}_{p} \in H_{0}^{1}\left(0, \infty ; \mathbf{H}\left(\Gamma_{i}\right)\right)$ satisfying $\left.\widehat{u}^{0}\right|_{\Gamma_{s}}=\eta_{2}^{0} n$ and

$$
\left\|\widehat{u}^{0}\right\|_{\mathbf{H}^{1}(\Omega)}+\left\|\eta_{2}^{0}\right\|_{H_{0}^{1}\left(\Gamma_{s}\right)}+\left\|\hat{g}_{p}\right\|_{H_{0}^{1}\left(0, \infty ; \mathbf{H}\left(\Gamma_{i}\right)\right)} \leq r
$$

we can find a control $\widehat{\mathbf{f}} \in H^{1}\left(0, \infty ; \mathbb{R}^{n_{c}}\right)$, in feedback form, for which the nonlinear system (3.3) admits a solution $\left(\widehat{u}, \widehat{p}, \widehat{\eta}_{1}, \widehat{\eta}_{2}\right) \in X_{\delta_{0}}$, where $\delta_{0}$ is introduced in (4.4), obeying

$$
\left\|e^{-\omega t} \widehat{\eta}_{1}(t)\right\|_{L^{\infty}\left(\Gamma_{s}\right)} \leq \eta_{\max } \quad \text { and } \quad\left\|\left(\widehat{u}(t), \widehat{\eta}(t), \widehat{\eta}_{t}(t)\right)\right\|_{\mathbf{H}^{\frac{3}{4}+\frac{\varepsilon_{0}}{2}}(\Omega) \times H^{3}\left(\Gamma_{s}\right) \times H^{1}\left(\Gamma_{s}\right)} \leq C, \quad \forall t>0,
$$

where $C>0$ depends on $r$ and $0<\varepsilon_{0}<\frac{1}{2}$.

REMARK 3.4. The estimate $\left\|e^{-\omega t} \widehat{\eta}_{1}(t)\right\|_{L^{\infty}\left(\Gamma_{s}\right)} \leq \eta_{\max }$ guarantees that, for all $t>0$, the change of variables $\mathcal{T}_{e^{-\omega t}} \widehat{\eta}(t)$ is a $\mathcal{C}^{1}$-diffeomorphism from $\Omega_{e^{-\omega t} \hat{\eta}(t)}$ into $\Omega$.

REMARK 3.5. In Theorems 3.2 and 3.3, we assume that $\eta_{1}(0)$, the initial displacement of the beam, is zero, see (3.3). As in [24], the initial displacement can be considered to be non zero. In that case, the compatibility conditions are more complicated (see [24] for compatibility conditions stated when $\left.\eta_{1}(0) \neq 0\right)$.

4. The Oseen system. The goal of this section is to study the non-homogeneous Oseen system

$$
\begin{aligned}
& \lambda_{0} w-\operatorname{div} \sigma(w, \pi)+\left(u_{s} \cdot \nabla\right) w+(w \cdot \nabla) u_{s}=F, \quad \operatorname{div} w=h \text { in } \Omega, \\
& w=\eta n \text { on } \Gamma_{s}, \quad w=0 \text { on } \Gamma_{i}, \quad w \cdot n=0 \text { and } \varepsilon(w) n \cdot \tau=0 \text { on } \Gamma_{e}, \quad \sigma(w, \pi) n=0 \text { on } \Gamma_{n},
\end{aligned}
$$

where $F \in \mathbf{L}^{2}(\Omega), \eta \in H_{0}^{\frac{3}{2}}\left(\Gamma_{s}\right), h \in H^{1}(\Omega)$. We choose $\lambda_{0}>0$ such that

$$
\lambda_{0} \int_{\Omega}|v|^{2}+2 \nu \int_{\Omega}|\varepsilon(v)|^{2}+\int_{\Omega}\left[\left(u_{s} \cdot \nabla\right) v+(v \cdot \nabla) u_{s}\right] \cdot v \geq \frac{\nu}{2}\|v\|_{\mathbf{H}^{1}(\Omega)}, \quad \forall v \in \mathbf{H}^{1}(\Omega) .
$$

We shall say that $(w, \pi) \in \mathbf{H}^{1}(\Omega) \times L^{2}(\Omega)$ is a weak solution of (4.1) if and only if it satisfies the following mixed variational formulation

Find $(w, \pi) \in \mathbf{H}^{1}(\Omega) \times L^{2}(\Omega)$ such that

$$
a(w, \phi)-b(\phi, \pi)=\int_{\Omega} F \cdot \phi \quad \text { for all } \phi \in \mathbf{H}_{\Gamma_{0}}^{1}(\Omega), \quad b(w, \psi)=\int_{\Omega} h \psi \quad \text { for all } \psi \in L^{2}(\Omega),
$$

$$
w=\eta n \text { on } \Gamma_{s}, \quad w=0 \text { on } \Gamma_{i}, \quad w \cdot n=0 \text { on } \Gamma_{e},
$$

where

$$
a(w, \phi)=\int_{\Omega}\left(\lambda_{0} w \phi+2 \nu \varepsilon(w): \varepsilon(\phi)\right) d x+\int_{\Omega}\left(\left(u_{s} \cdot \nabla\right) w+(w \cdot \nabla) u_{s}\right) \phi d x, \quad b(w, \psi)=\int_{\Omega} \operatorname{div}(w) \psi d x .
$$


In the case when $u_{s}=0$, that is for the Stokes system, if $(F, h, \eta) \in \mathbf{L}^{2}(\Omega) \times H^{1}(\Omega) \times H_{0}^{\frac{3}{2}}\left(\Gamma_{s}\right)$, far from the set $\mathcal{C}$, that is in $\Omega \backslash \cup_{j=1,8} B\left(C_{j}, \varepsilon\right)$, the solution $(w, \pi)$ to system (4.1) belongs to $H^{2}(\Omega \backslash$ $\left.\cup_{j=1,8} B\left(C_{j}, \varepsilon\right)\right) \times H^{1}\left(\Omega \backslash \cup_{j=1,8} B\left(C_{j}, \varepsilon\right)\right)$ for all $\varepsilon>0\left(B\left(C_{j}, \varepsilon\right)\right.$ is the ball centered at $C_{j}$ of radius $\left.\varepsilon\right)$. In the neighborhood of $\mathcal{C}$, we can study the regularity of the solution to system (4.1) in weighted Sobolev spaces. According to [20, Theorem 9.4.5], there exists $\delta_{0}<1 / 2$ such that, if $(F, h, \eta) \in \mathbf{L}^{2}(\Omega) \times H^{1}(\Omega) \times$ $H_{0}^{\frac{3}{2}}\left(\Gamma_{s}\right)$ and $u_{s}=0$, the solution $(w, \pi)$ to system (4.1) belongs to $\mathbf{H}_{\delta_{0}}^{2}(\Omega) \times H_{\delta_{0}}^{1}(\Omega)$. In particular $\delta_{0}<1 / 2$ can be chosen so that

$$
\frac{1}{3}<\delta_{0}<\frac{1}{2}
$$

The regularity result for the Oseen system is stated in the following theorem.

THEOREM 4.1. For all $(F, h, \eta) \in \mathbf{L}^{2}(\Omega) \times H^{1}(\Omega) \times H_{0}^{\frac{3}{2}}\left(\Gamma_{s}\right)$, system (4.1) admits a unique solution $(w, \pi) \in \mathbf{H}_{\delta_{0}}^{2}(\Omega) \times H_{\delta_{0}}^{1}(\Omega)$ satisfying the estimate

$$
\|w\|_{\mathbf{H}_{\delta_{0}}^{2}(\Omega)}+\|\pi\|_{H_{\delta_{0}}^{1}(\Omega)} \leq C\left(\|F\|_{\mathbf{L}^{2}(\Omega)}+\|h\|_{H^{1}(\Omega)}+\|\eta\|_{H^{3 / 2}\left(\Gamma_{s}\right)}\right) .
$$

Proof. As mentioned above, if $u_{s}=0$, the existence of a unique solution to system (4.1) belonging to $\mathbf{H}_{\delta_{0}}^{2}(\Omega) \times H_{\delta_{0}}^{1}(\Omega)$ is already proved in [20, Theorem 9.4.5]. However, we have to notice that the variational formulation (4.3) is different from that in [20, (9.1.7)-(9.1.9)]. It is different because equation $(4.1)_{1}$ is written in terms of $-\operatorname{div} \sigma(w, \pi)$, while in [20] equation (9.1.1) is written in terms of $-\nu \Delta w+\nabla p$. But the results stated in $[20$, Theorem 9.4.5] can be applied here to the Oseen system stated in (4.1).

The adaptation from the Stokes system to the Oseen system can be performed as in [21, Proof of Theorem 2.17, Step 2].

REMARK 4.2. If in system (4.1) the boundary condition $w=0$ on $\Gamma_{i}$ is replaced by $w=g_{p}$ on $\Gamma_{i}$, we can prove that, for $\left(F, h, \eta, g_{p}\right) \in \mathbf{L}^{2}(\Omega) \times H^{1}(\Omega) \times H_{0}^{\frac{3}{2}}\left(\Gamma_{s}\right) \times \mathbf{H}\left(\Gamma_{i}\right)$, the solution to system (4.1) satisfies

$$
\|w\|_{\mathbf{H}_{\delta_{0}}^{2}(\Omega)}+\|\pi\|_{H_{\delta_{0}}^{1}(\Omega)} \leq C\left(\|F\|_{\mathbf{L}^{2}(\Omega)}+\|h\|_{H^{1}(\Omega)}+\|\eta\|_{H^{3 / 2}\left(\Gamma_{s}\right)}+\left\|g_{p}\right\|_{\mathbf{H}\left(\Gamma_{i}\right)}\right) .
$$

Lemma 4.3. We set $\varepsilon_{0}=\frac{1}{2}-\delta_{0}$. Then $\varepsilon_{0}>0, \mathbf{H}_{\delta_{0}}^{2}(\Omega) \subset \mathbf{H}^{\frac{3}{2}+\varepsilon_{0}}(\Omega)$ and $H_{\delta_{0}}^{1}(\Omega) \subset H^{\frac{1}{2}+\varepsilon_{0}}(\Omega)$, with continuous embeddings.

Proof. It follows from [20, Lemma 6.2.1].

4.1. The Leray projector. From $\left[21\right.$, Lemma 2.2], we know that $\mathbf{L}^{2}(\Omega)=\mathbf{V}_{n, \Gamma_{0}}^{0}(\Omega) \oplus \nabla H_{\Gamma_{n}}^{1}(\Omega)$, where $H_{\Gamma_{n}}^{1}(\Omega)=\left\{p \in H^{1}(\Omega)|p|_{\Gamma_{n}}=0\right\}$. We introduce the orthogonal projection $P$ from $\mathbf{L}^{2}(\Omega)$ onto $\mathbf{V}_{n, \Gamma_{0}}^{0}(\Omega)$, called the Leray projector. For all $v$ in $\mathbf{L}^{2}(\Omega)$, we have

$$
P v=v-\nabla q_{v}^{1}-\nabla q_{v}^{2}
$$

where $q_{v}^{1}$ and $q_{v}^{2}$ are the solutions to the equations

$$
\begin{aligned}
& q_{v}^{1} \in H_{0}^{1}(\Omega), \quad \Delta q_{v}^{1}=\operatorname{div} v \text { in } \Omega, \quad q_{v}^{1}=0 \text { on } \partial \Omega, \\
& q_{v}^{2} \in H^{1}(\Omega), \quad \Delta q_{v}^{2}=0 \text { in } \Omega, \quad \frac{\partial q_{v}^{2}}{\partial n}=\left(v-\nabla q_{v}^{1}\right) \cdot n \text { on } \Gamma_{0}, \quad q_{v}^{2}=0 \text { on } \Gamma_{n} .
\end{aligned}
$$

Proposition 4.4. If $v$ belongs to $\mathbf{H}^{1}(\Omega)$, then $P v$ and $(I-P) v$ belong to $\mathbf{H}^{\frac{1}{2}+\varepsilon_{0}}(\Omega)$. Moreover, we have $(I-P) v=\nabla q$, where $q$ is the solution to the equation

$$
q \in H^{1}(\Omega), \quad \Delta q=\operatorname{div} v \text { in } \Omega, \quad \frac{\partial q}{\partial n}=v \cdot n \text { on } \Gamma_{0}, \quad q=0 \text { on } \Gamma_{n} .
$$

Proof. We have to prove that the solution $q$ to equation (4.6) belongs to $H^{\frac{3}{2}+\varepsilon_{0}}(\Omega)$. Since $v$ belongs to $\mathbf{H}^{1}(\Omega)$, we know that $\left.v \cdot n\right|_{\Gamma_{0}}$ belongs to $H^{\frac{1}{2}}\left(\Gamma_{0}\right)$. Thus, far from the corners of the domain $\Omega$, the solution $q$ is of class $H^{2}$. We have to analyze the regularity at the corners corresponding either to a 
junction between two Neumann boundary conditions or to a junction between a Neumann and Dirichlet boundary condition. At the two corners corresponding to a Neumann-Dirichlet junction, the angle is equal to $\frac{\pi}{2}$, the condition on $\Gamma_{n}$ is $q=0$ and the condition on $\Gamma_{0}$ is $\frac{\partial q}{\partial n} \in H^{\frac{1}{2}}\left(\Gamma_{0}\right)$. Using an odd symmetry with respect to $\Gamma_{0}$, we have to deal with a pure Neumann boundary condition on a flat boundary with the extension of $\frac{\partial q}{\partial n}$ belonging to $H^{\frac{1}{2}-\varepsilon}$ for all $\varepsilon>0$, on that flat boundary. Thus $q$ is of class $H^{2-\varepsilon}$, for all $\varepsilon>0$, close to a Neumann-Dirichlet junction.

We may use the results in [12] to analyze the regularity at the corners corresponding to a NeumannNeumann junction. From [12, Corollary 2.4.4, Remark 2.4.6, and Theorem 2.3.7 (iii)], it follows that $q \in H^{s}(\Omega)$ for all $s<1+\frac{2}{3}$. In particular $q \in H^{\frac{3}{2}+\varepsilon_{0}}(\Omega)$. This completes the proof.

4.2. Expression of the pressure. The goal of this section is to express the pressure $\pi$ in equation (4.1) in terms of $w, F, \eta$ and $h$. The method used in [24] consists in calculating the divergence of equation $(4.1)_{1}$ in order to get an elliptic equation for $\pi$. This method does not work here. Indeed, formally $\pi$ is the solution to the equation

$$
\begin{aligned}
& \Delta \pi=-\lambda_{0} h-\operatorname{div}\left(\left(u_{s} \cdot \nabla\right) w+(w \cdot \nabla) u_{s}\right)+\operatorname{div} F \text { in } \Omega, \quad \pi=2 \nu \varepsilon(w) n \cdot n \text { on } \Gamma_{n}, \\
& \frac{\partial \pi}{\partial n}=2 \nu \operatorname{div} \varepsilon(w) \cdot n-\lambda_{0} w \cdot n-\left(\left(u_{s} \cdot \nabla\right) w+(w \cdot \nabla) u_{s}\right) \cdot n+F \cdot n \text { on } \Gamma_{0} .
\end{aligned}
$$

Since $\operatorname{div} \varepsilon(w) \notin \mathbf{L}^{2}(\Omega)$, the boundary condition $(4.7)_{2}$ is not well-posed. Thus, we cannot use the classical transposition method because the Green formula needed for that is not valid (see [12, Theorem 1.5.3]). We are going to use a variant of the classical transposition method to define an equation for the pressure $\pi$. First, we consider the equation

$$
\Delta \chi=\zeta \text { in } \Omega, \quad \frac{\partial \chi}{\partial n}=0 \text { on } \Gamma_{0}, \quad \chi=0 \text { on } \Gamma_{n} .
$$

Lemma 4.5. For all $\zeta \in L^{2}(\Omega)$, equation (4.8) admits a unique solution $\chi$ belonging to $H_{\delta}^{2}(\Omega)$, for all $\frac{1}{3}<\delta<1$, and satisfying

$$
\|\chi\|_{H_{\delta}^{2}(\Omega)} \leq C_{\delta}\|\zeta\|_{L^{2}(\Omega)},
$$

where $C_{\delta}$ depends on $\delta$. In particular, this estimate is valid for $\delta=\delta_{0}$.

Proof. It follows from [20, Theorem 6.5.4], [20, Lemma 6.2.1] and [15, Chapter 2].

Let us notice that

$$
H_{\delta}^{1}(\Omega) \hookrightarrow L_{-\delta}^{2}(\Omega) \quad \text { for all } 0<\delta<\frac{1}{2} .
$$

Indeed, from [19, page 399], it follows that $H_{\delta}^{1}(\mathcal{D}) \hookrightarrow H_{-\delta}^{\varepsilon}(\mathcal{D})$ for a dihedral domain $\mathcal{D}$, when $1-\delta=\varepsilon+\delta$ and $\varepsilon>0$. Thus we have $H_{\delta}^{1}(\Omega) \hookrightarrow H_{-\delta}^{\varepsilon}(\Omega)$ for all $0<\delta<\frac{1}{2}$ and $\varepsilon=1-2 \delta$. That proves (4.9), since $H_{-\delta}^{\varepsilon}(\Omega) \hookrightarrow L_{-\delta}^{2}(\Omega)$.

Using (4.9), we can define $k \in \mathcal{L}\left(L^{2}(\Omega), \mathbb{R}\right)$ by

$$
\begin{aligned}
k(\zeta)= & \lambda_{0} \int_{\Gamma_{s}} \eta \chi-\lambda_{0} \int_{\Omega} h \chi-\int_{\Omega} F \cdot \nabla \chi+2 \nu\left\langle\varepsilon(w), \nabla^{2} \chi\right\rangle_{\mathbf{L}_{-\delta_{0}}^{2}}(\Omega), \mathbf{L}_{\delta_{0}}^{2}(\Omega) \\
& -2 \nu \int_{\Gamma_{0}} \varepsilon(w) n \cdot \nabla \chi+\int_{\Omega}\left[\left(u_{s} \cdot \nabla\right) w+(w \cdot \nabla) u_{s}\right] \cdot \nabla \chi, \quad \text { for all } \zeta \in L^{2}(\Omega),
\end{aligned}
$$

where $\chi \in H_{\delta_{0}}^{2}(\Omega)$ is the solution to equation (4.8).

Lemma 4.6. Let $(w, F, \eta, h)$ belong to $\mathbf{H}_{\delta_{0}}^{2}(\Omega) \times \mathbf{L}^{2}(\Omega) \times L^{2}\left(\Gamma_{s}\right) \times H^{1}(\Omega)$. The operator $k$ belongs to $\mathcal{L}\left(L^{2}(\Omega), \mathbb{R}\right)$ and we have

$$
|k(\zeta)| \leq C\left(\|\eta\|_{L^{2}\left(\Gamma_{s}\right)}+\|h\|_{L^{2}(\Omega)}+\|F\|_{\mathbf{L}^{2}(\Omega)}+\|w\|_{\mathbf{H}_{\delta_{0}}^{2}(\Omega)}\right)\|\zeta\|_{L^{2}(\Omega)} \quad \text { for all } \zeta \in L^{2}(\Omega) .
$$


Proof. The lemma follows from the following estimates

$$
\begin{aligned}
& \left|\int_{\Gamma_{s}} \eta \chi\right| \leq C\|\eta\|_{L^{2}\left(\Gamma_{s}\right)}\|\chi\|_{L^{2}\left(\Gamma_{s}\right)} \leq C\|\eta\|_{L^{2}\left(\Gamma_{s}\right)}\|\zeta\|_{L^{2}(\Omega)} \\
& \left|\int_{\Omega} h \chi\right| \leq C\|h\|_{L^{2}(\Omega)}\|\chi\|_{L^{2}(\Omega)} \leq C\|h\|_{L^{2}(\Omega)}\|\zeta\|_{L^{2}(\Omega)}, \\
& \left|\int_{\Omega} F \cdot \nabla \chi\right| \leq C\|F\|_{\mathbf{L}^{2}(\Omega)}\|\nabla \chi\|_{L^{2}(\Omega)} \leq C\|F\|_{\mathbf{L}^{2}(\Omega)}\|\zeta\|_{L^{2}(\Omega)}, \\
& \left|\int_{\Gamma_{0}} \varepsilon(w) n \cdot \nabla \chi\right| \leq C\|\varepsilon(w)\|_{\mathbf{L}^{2}\left(\Gamma_{0}\right)}\|\nabla \chi\|_{\mathbf{L}^{2}\left(\Gamma_{0}\right)} \leq C\|w\|_{\mathbf{H}^{\frac{3}{2}+\varepsilon_{0}(\Omega)}}\|\zeta\|_{L^{2}(\Omega)}, \\
& \left|\int_{\Omega}\left[\left(u_{s} \cdot \nabla\right) w+(w \cdot \nabla) u_{s}\right] \cdot \nabla \chi\right| \leq C\|w\|_{\mathbf{H}^{1}(\Omega)}\|\nabla \chi\|_{\mathbf{L}^{2}(\Omega)} \leq C\|w\|_{\mathbf{H}^{1}(\Omega)}\|\zeta\|_{L^{2}(\Omega)}, \\
& \left|\left\langle\varepsilon(w), \nabla^{2} \chi\right\rangle_{\mathbf{L}_{-\delta_{0}}^{2}(\Omega), \mathbf{L}_{\delta_{0}}^{2}(\Omega)}\right| \leq\left. C\|\varepsilon(w)\|\right|_{\mathbf{L}_{-\delta_{0}}^{2}(\Omega)}\left\|\nabla^{2} \chi\right\|_{\mathbf{L}_{\delta_{0}}^{2}(\Omega)} \leq C\|w\|_{\mathbf{H}^{1+\delta_{0}}(\Omega)}\|\zeta\|_{H_{\delta_{0}}^{2}(\Omega)} .
\end{aligned}
$$

The last inequality comes from [20, Lemma 6.2.1].

We introduce the following operators:

- $N_{s} \in \mathcal{L}\left(L^{2}\left(\Gamma_{s}\right), H^{1}(\Omega)\right)$ is defined by $N_{s} \eta=q$ where $q$ is the solution to the equation

$$
\Delta q=0 \text { in } \Omega, \quad \frac{\partial q}{\partial n}=\eta \text { on } \Gamma_{s}, \quad \frac{\partial q}{\partial n}=0 \text { on } \Gamma_{0} \backslash \Gamma_{s}, \quad q=0 \text { on } \Gamma_{n} .
$$

- $N_{\text {div }} \in \mathcal{L}\left(L^{2}(\Omega), H^{1}(\Omega)\right)$ is defined by $N_{\text {div }} h=q$ where $q$ is the solution to the equation

$$
\Delta q=h \text { in } \Omega, \quad \frac{\partial q}{\partial n}=0 \text { on } \Gamma_{0}, \quad q=0 \text { on } \Gamma_{n} .
$$

- $N_{p} \in \mathcal{L}\left(\mathbf{L}^{2}(\Omega), H^{1}(\Omega)\right)$ is defined by $N_{p} F=q_{F}^{1}+q_{F}^{2}$ where $q_{F}^{1}$ and $q_{F}^{2}$ are the solutions to equations $(4.5)_{1}$ and $(4.5)_{2}$ respectively, with $v=F$.

- $N_{v} \in \mathcal{L}\left(\mathbf{H}_{\delta_{0}}^{2}(\Omega), L^{2}(\Omega)\right)$ is defined by $N_{v} w=q$ where $q$ is the solution to the variational problem

Find $q \in L^{2}(\Omega)$ such that

$$
\int_{\Omega} q \zeta=2 \nu\left\langle\varepsilon(w), \nabla^{2} \chi\right\rangle_{\mathbf{L}_{-\delta_{0}}^{2}(\Omega), \mathbf{L}_{\delta_{0}}^{2}(\Omega)}-2 \nu \int_{\Gamma_{0}} \varepsilon(w) n \cdot \nabla \chi+\int_{\Omega}\left[\left(u_{s} \cdot \nabla\right) w+(w \cdot \nabla) u_{s}\right] \cdot \nabla \chi,
$$

for all $\zeta \in L^{2}(\Omega)$, where $\chi \in H_{\delta_{0}}^{2}(\Omega)$ is the solution to equation (4.8).

Thanks to the Lax-Milgram Theorem, we can easily prove that the operators $N_{s}, N_{\text {div }}, N_{p}$ and $N_{v}$ are well-defined.

REMARK 4.7. We notice that $\nabla N_{p}=I-P$.

THEOREM 4.8. If $(w, \pi) \in \mathbf{H}_{\delta_{0}}^{2}(\Omega) \times H_{\delta_{0}}^{1}(\Omega)$ is a solution to system (4.1), where $\delta_{0}$ obeys (4.4), then $\pi$ is the unique solution to the problem

$$
\text { Find } \pi \in L^{2}(\Omega) \text { such that } \int_{\Omega} \pi \zeta d x=k(\zeta), \quad \forall \zeta \in L^{2}(\Omega) .
$$

As a consequence, $\pi$ is determined by

$$
\pi=-\lambda_{0} N_{s} \eta-\lambda_{0} N_{\text {div }} h+N_{p} F+N_{v} w .
$$

Proof. Let $(w, \pi) \in \mathbf{H}_{\delta_{0}}^{2}(\Omega) \times H_{\delta_{0}}^{1}(\Omega)$ be the solution to system (4.1) and let $\zeta$ belong to $L^{2}(\Omega)$. Multiplying the first equation of the system by $\nabla \chi$, where $\chi$ is the solution to equation (4.8), we obtain

$$
\lambda_{0} \int_{\Omega} w \cdot \nabla \chi-\int_{\Omega} \operatorname{div} \sigma(w, \pi) \cdot \nabla \chi+\int_{\Omega}\left[\left(u_{s} \cdot \nabla\right) w+(w \cdot \nabla) u_{s}\right] \cdot \nabla \chi-\int_{\Omega} F \cdot \nabla \chi=0 .
$$

By using integration by parts, we get

$$
\lambda_{0} \int_{\Omega} w \cdot \nabla \chi=-\lambda_{0} \int_{\Omega} \operatorname{div} w \cdot \chi+\int_{\partial \Omega} w \cdot n \chi=-\lambda_{0} \int_{\Omega} h \chi+\lambda_{0} \int_{\Gamma_{s}} \eta \chi,
$$


and

$$
\begin{aligned}
\int_{\Omega} \operatorname{div} \sigma(w, \pi) \cdot \nabla \chi & =-\left\langle\sigma(w, \pi), \nabla^{2} \chi\right\rangle_{\mathbf{L}_{-\delta_{0}}^{2}}(\Omega), \mathbf{L}_{\delta_{0}}^{2}(\Omega) \\
& =-2 \nu\left\langle\varepsilon(w), \int_{\partial \Omega}^{2} \chi\right\rangle_{\mathbf{L}_{-\delta_{0}}^{2}(\Omega), \mathbf{L}_{\delta_{0}}^{2}(\Omega)} \sigma(w, \pi) n \cdot \nabla \chi \\
& \pi \cdot \zeta+\int_{\Gamma_{0}} \sigma(w, \pi) n \cdot \nabla \chi .
\end{aligned}
$$

Let us notice that (4.16) $)_{1}$ can be first obtained for $\chi \in C^{\infty}(\bar{\Omega})$ and next by density for $\chi \in \mathbf{H}_{\delta_{0}}^{2}(\Omega)$. From equations (4.14)-(4.16), it follows that $\pi$ is a solution of equation (4.13). Thanks to Lemma 4.6 and to the Lax-Milgram Lemma in $L^{2}(\Omega)$, we prove that (4.13) admits a unique solution.

To prove the last part of the theorem, it is sufficient to verify that

$$
\begin{aligned}
& \int_{\Gamma_{s}} \eta \chi=\int_{\Gamma_{s}} \frac{\partial N_{s} \eta}{\partial n} \chi=-\int_{\Omega} \zeta N_{s} \eta \\
& \int_{\Omega} h \chi=\int_{\Omega} \Delta N_{\mathrm{div}} h \chi=\int_{\Omega} \zeta N_{\mathrm{div}} h \text { and } \int_{\Omega} F \cdot \nabla \chi=\int_{\Omega} \zeta N_{p} F .
\end{aligned}
$$

Lemma 4.9. Let $(w, \pi) \in \mathbf{H}_{\delta_{0}}^{2}(\Omega) \times H_{\delta_{0}}^{1}(\Omega)$ be the solution of system (4.1), where $\delta_{0}$ obeys (4.4). Then $N_{v} w$ belongs to $H^{\frac{1}{2}+\varepsilon_{0}}(\Omega)$.

Proof. We start from the identity

$$
\pi=-\lambda_{0} N_{s} \eta-\lambda_{0} N_{\mathrm{div}} h+N_{p} F+N_{v} w .
$$

We know that $N_{p} F, N_{\text {div }} h$ and $N_{s} \eta$ belong to $H^{1}(\Omega)$ and that $\pi$ belongs to $H_{\delta_{0}}^{1}(\Omega) \subset H^{\frac{1}{2}+\varepsilon_{0}}(\Omega)$ (see Lemma 4.3). Hence $N_{v} w$ belongs to $H^{\frac{1}{2}+\varepsilon_{0}}(\Omega)$.

4.3. The Oseen operator. We introduce the Oseen operator $(A, D(A))$ in $\mathbf{V}_{n, \Gamma_{0}}^{0}(\Omega)$ defined by

$$
\begin{gathered}
D(A)=\left\{v \in \mathbf{V}_{n, \Gamma_{0}}^{\frac{3}{2}+\varepsilon_{0}}(\Omega) \mid \exists q \in H^{\frac{1}{2}+\varepsilon_{0}}(\Omega) \text { such that } \operatorname{div} \sigma(v, q) \in \mathbf{L}^{2}(\Omega),\right. \\
\left.\varepsilon(v) n \cdot \tau=0 \text { on } \Gamma_{e}, \sigma(v, q) n=0 \text { on } \Gamma_{n}\right\} \text { and } \\
A v=P \operatorname{div} \sigma(v, q)-P\left[\left(u_{s} \cdot \nabla\right) v+(v \cdot \nabla) u_{s}\right] .
\end{gathered}
$$

THEOREM 4.10. The operator $(A, D(A))$ is the infinitesimal generator of an analytic semigroup on $\mathbf{V}_{n, \Gamma_{0}}^{0}(\Omega)$ and its resolvent is compact.

Proof. The proof is similar to that of [21, Theorem 2.8].

Proposition 4.11. The adjoint of $(A, D(A))$ in $\mathbf{V}_{n, \Gamma_{0}}^{0}(\Omega)$ is defined by

$$
\begin{gathered}
D\left(A^{*}\right)=\left\{v \in \mathbf{V}_{n, \Gamma_{0}}^{\frac{3}{2}+\varepsilon_{0}}(\Omega) \mid \exists q \in H^{\frac{1}{2}+\varepsilon_{0}}(\Omega) \text { such that } \operatorname{div} \sigma(v, q) \in \mathbf{L}^{2}(\Omega),\right. \\
\left.\varepsilon(v) n \cdot \tau=0 \text { on } \Gamma_{e}, \sigma(v, q) n+u_{s} \cdot n v=0 \text { on } \Gamma_{n}\right\} \text { and } \\
A^{*} v=P \operatorname{div} \sigma(v, p)+P\left(u_{s} \cdot \nabla\right) v-P\left(\nabla u_{s}\right)^{T} v .
\end{gathered}
$$

Proof. See [21, Theorem 2.11].

Finally, we introduce the lifting operators $L \in \mathcal{L}\left(H_{0}^{\frac{3}{2}}\left(\Gamma_{s}\right) \times H^{1}(\Omega), \mathbf{H}_{\delta_{0}}^{2}(\Omega)\right)$ and $L_{p} \in \mathcal{L}\left(H_{0}^{\frac{3}{2}}\left(\Gamma_{s}\right) \times\right.$ $\left.H^{1}(\Omega), H_{\delta_{0}}^{1}(\Omega)\right)$ defined by

$$
L(\eta, h)=w \quad \text { and } \quad L_{p}(\eta, h)=\pi,
$$

where $(w, \pi)$ is the solution to system (4.1) for $F=0$.

TheOrem 4.12. A pair $(w, \pi) \in \mathbf{H}_{\delta_{0}}^{2}(\Omega) \times H_{\delta_{0}}^{1}(\Omega)$ is solution to system (4.1) if and only if

$$
\begin{aligned}
& \left(\lambda_{0} I-A\right) P w+\left(A-\lambda_{0} I\right) P L(\eta, h)=P F, \quad(I-P) w=\nabla N_{s} \eta+\nabla N_{\mathrm{div}} h, \\
& \pi=-\lambda_{0} N_{s} \eta-\lambda_{0} N_{\mathrm{div}} h+N_{p} F+N_{v} w .
\end{aligned}
$$


Proof. Let $(w, \pi) \in \mathbf{H}_{\delta_{0}}^{2}(\Omega) \times H_{\delta_{0}}^{1}(\Omega)$ be a solution of system (4.1). We set

$$
\widehat{w}=w-L(\eta, h) \quad \text { and } \quad \widehat{\pi}=\pi-L_{p}(\eta, h) .
$$

The couple $(\widehat{w}, \widehat{\pi})$ is solution to system $(4.1)$ with $(\eta, h)=(0,0)$. Thus, $\widehat{w}$ belongs to $D(A)$ and $\lambda_{0} P \widehat{w}-$ $A P \widehat{w}=P F$. Hence $\left(\lambda_{0} I-A\right) P w+\left(A-\lambda_{0} I\right) P L(\eta, h)=P F$. The last two equations in (4.20) come from Proposition 4.4 and Theorem 4.8 .

Now, we suppose that $(w, \pi) \in \mathbf{H}_{\delta_{0}}^{2}(\Omega) \times H_{\delta_{0}}^{1}(\Omega)$ is solution to system (4.20). Since $(I-P) w=$ $\nabla N_{s} \eta+\nabla N_{\text {div }} h=(I-P) L(\eta, h)$, we have

$$
\widehat{w}=w-L(\eta, h) \in D(A) .
$$

Thus, there exists $\pi_{1} \in H^{\frac{1}{2}+\varepsilon_{0}}(\Omega)$ such that

$$
A \widehat{w}=P \operatorname{div} \sigma\left(\widehat{w}, \pi_{1}\right)-P\left(u_{s} \cdot \nabla\right) \widehat{w}-P(\widehat{w} \cdot \nabla) u_{s} \quad \text { and } \quad \sigma\left(\widehat{w}, \pi_{1}\right) n=0 \text { on } \Gamma_{n} .
$$

From the first equation of system (4.20), it follows that

$$
P\left[\lambda_{0} \widehat{w}-\operatorname{div} \sigma\left(\widehat{w}, \pi_{1}\right)+\left(u_{s} \cdot \nabla\right) \widehat{w}+(\widehat{w} \cdot \nabla) u_{s}-F\right]=0 .
$$

Thus, there exists $\pi_{2} \in H_{\Gamma_{n}}^{1}(\Omega)$ such that

$$
\lambda_{0} \widehat{w}-\operatorname{div} \sigma\left(\widehat{w}, \pi_{1}+\pi_{2}\right)+\left(u_{s} \cdot \nabla\right) \widehat{w}+(\widehat{w} \cdot \nabla) u_{s}=F .
$$

With equations (4.21), $(4.22)_{2}$ and (4.23), we obtain the system

$$
\begin{aligned}
& \lambda_{0} \widehat{w}-\operatorname{div} \sigma\left(\widehat{w}, \pi_{1}+\pi_{2}\right)+\left(u_{s} \cdot \nabla\right) \widehat{w}+(\widehat{w} \cdot \nabla) u_{s}=F, \quad \operatorname{div} \widehat{w}=0 \text { in } \Omega, \\
& \widehat{w}=0 \text { on } \Gamma_{s}, \quad \widehat{w}=0 \text { on } \Gamma_{i}, \quad \widehat{w} \cdot n=0 \text { and } \varepsilon(\widehat{w}) n \cdot \tau=0 \text { on } \Gamma_{e}, \quad \sigma\left(\widehat{w}, \pi_{1}+\pi_{2}\right) n=0 \text { on } \Gamma_{n} .
\end{aligned}
$$

We deduce that $(w, \pi)$, with

$$
\pi=\pi_{1}+\pi_{2}+L_{p}(\eta, h)=-\lambda_{0} N_{s} \eta-\lambda_{0} N_{\text {div }} h+N_{p} F+N_{v} w,
$$

is the solution to system (4.1).

5. Reformulation of the linearized system. For all $\left(F_{f}, F_{s}^{1}, F_{s}^{2}\right)$ belonging to $H=\mathbf{L}^{2}(\Omega) \times$ $H_{0}^{2}\left(\Gamma_{s}\right) \times L^{2}\left(\Gamma_{s}\right)$, we consider the system

$$
\begin{aligned}
& \lambda v-\operatorname{div} \sigma(v, q)+\left(u_{s} \cdot \nabla\right) v+(v \cdot \nabla) u_{s}-A_{1} \eta_{1}-A_{2} \eta_{2}=F_{f}, \quad \operatorname{div} v=A_{3} \eta_{1} \text { in } \Omega, \\
& v=\eta_{2} n \text { on } \Gamma_{s}, \quad v=0 \text { on } \Gamma_{i}, \quad v \cdot n=0 \text { and } \varepsilon(v) n \cdot \tau=0 \text { on } \Gamma_{e}, \quad \sigma(v, q) n=0 \text { on } \Gamma_{n}, \\
& \lambda \eta_{1}-\eta_{2}=F_{s}^{1} \text { on } \Gamma_{s}, \\
& \lambda \eta_{2}-\beta \Delta_{s} \eta_{1}-\gamma \Delta_{s} \eta_{2}+\alpha \Delta_{s}^{2} \eta_{1}-A_{4} \eta_{1}=\gamma_{s} q+F_{s}^{2} \text { on } \Gamma_{s}, \\
& \eta_{1}=0 \text { on } \partial \Gamma_{s}, \quad \eta_{1, x}=0 \text { on } \partial \Gamma_{s} .
\end{aligned}
$$

\subsection{Properties of the operators $A_{1}, A_{2}, A_{3}$ and $A_{4}$.}

Proposition 5.1. The differential operators $A_{1}, A_{2}, A_{3}$ and $A_{4}$ obey

$$
\begin{aligned}
& A_{1} \in \mathcal{L}\left(H_{0}^{2}\left(\Gamma_{s}\right), \mathbf{L}^{2}(\Omega)\right), \quad A_{2} \in \mathcal{L}\left(L^{2}\left(\Gamma_{s}\right), \mathbf{L}^{2}(\Omega)\right), \\
& A_{3} \in \mathcal{L}\left(H_{0}^{2}\left(\Gamma_{s}\right), H^{1}(\Omega)\right) \quad \text { and } \quad A_{4} \in \mathcal{L}\left(H_{0}^{2}\left(\Gamma_{s}\right), H^{\varepsilon_{0}}\left(\Gamma_{s}\right)\right) .
\end{aligned}
$$

Proof. For $A_{1}, A_{2}$ and $A_{3}$, the proposition follows from Lemmas 5.2 and 5.3 below, and for $A_{4}$, it follows from [13, Proposition B.1].

Lemma 5.2. There exists $C>0$ such that, for all $(\eta, w)$ belonging to $L^{2}\left(\Gamma_{s}\right) \times \mathbf{H}^{\frac{1}{2}+\varepsilon_{0}}(\Omega), \eta w$ belongs to $\mathbf{L}^{2}(\Omega)$ and

$$
\|\eta w\|_{\mathbf{L}^{2}(\Omega)} \leq C\|\eta\|_{L^{2}\left(\Gamma_{s}\right)}\|w\|_{\mathbf{H}^{\frac{1}{2}+\varepsilon_{0}(\Omega)}} .
$$


Proof. Thanks to the continuous embeddings $\mathbf{H}^{\frac{1}{2}+\varepsilon_{0}}\left(( \pm e, \pm \ell) \times\left(0, \ell_{s}\right)\right) \hookrightarrow L^{2}\left( \pm e, \pm \ell ; L^{\infty}\left(0, \ell_{s}\right)\right)$, and using the continuity of the extension operator $\mathcal{E}$ (see Remark 3.1), it follows that

$$
\begin{aligned}
\int_{\Omega}|\eta w|^{2} & \leq C \int_{-\ell}^{-e} \int_{0}^{\ell_{s}}|\eta(x,-e) w(x, z)|^{2} \mathrm{~d} x \mathrm{~d} z+C \int_{e}^{\ell} \int_{0}^{\ell_{s}}|\eta(x, e) w(x, z)|^{2} \mathrm{~d} x \mathrm{~d} z \\
& \leq C\|\eta\|_{L^{2}\left(\Gamma_{s}\right)}^{2}\|w\|_{\mathbf{H}^{\frac{1}{2}+\varepsilon_{0}}(\Omega)}^{2} .
\end{aligned}
$$

Lemma 5.3. There exists $C>0$ such that, for all $(\eta, w)$ belonging to $H_{0}^{1}\left(\Gamma_{s}\right) \times \mathbf{L}_{\delta_{0}}^{2}(\Omega)$, $\eta w$ belongs to $\mathbf{L}^{2}(\Omega)$ and

$$
\|\eta w\|_{\mathbf{L}^{2}(\Omega)} \leq C\|\eta\|_{H_{0}^{1}\left(\Gamma_{s}\right)}\|w\|_{\mathbf{L}_{\delta_{0}}^{2}(\Omega)} .
$$

Proof. Let us denote by $\left(C_{j}^{ \pm}\right)_{1 \leq j \leq 2}$, the four corners corresponding to the boundary of $\Gamma_{s}$, and by $\left(x_{j}^{ \pm}, z_{j}^{ \pm}\right)$the coordinates of $C_{j}$. Here the exponent \pm refers to the upper and lower beams. Since $\eta^{ \pm}$ belongs to $H_{0}^{1}\left(\Gamma_{s}\right)$, using an appropriate corner, we have $\left|\eta^{ \pm}(x)\right|=\left|\int_{x_{j}^{ \pm}}^{x} \eta_{x}(s) \mathrm{d} s\right| \leq\left\|\eta_{x}\right\|_{L^{2}\left(\Gamma_{s}\right)}\left|x-x_{j}^{ \pm}\right|^{\frac{1}{2}}$. Hence

$$
\begin{aligned}
\int_{\Omega}\left|\eta^{ \pm} w\right|^{2} & \leq\left\|\eta_{x}\right\|_{L^{2}\left(\Gamma_{s}\right)}^{2} \int_{\Omega} \frac{\left|x-x_{j}^{ \pm}\right|}{\left(\left|x-x_{j}^{ \pm}\right|^{2}+\left|z-z_{j}^{ \pm}\right|^{2}\right)^{2 \delta_{0}}}\left|\left(\left|x-x_{j}^{ \pm}\right|^{2}+\left|z-z_{j}^{ \pm}\right|^{2}\right)^{\delta_{0}} w(x, z)\right|^{2} \mathrm{~d} x \mathrm{~d} z \\
& \leq\left\|\eta_{x}\right\|_{L^{2}\left(\Gamma_{s}\right)}^{2} \int_{\Omega}\left(\left|x-x_{j}^{ \pm}\right|^{2}+\left|z-z_{j}^{ \pm}\right|^{2}\right)^{1-2 \delta_{0}}\left|\left(\left|x-x_{j}^{ \pm}\right|^{2}+\left|z-z_{j}^{ \pm}\right|^{2}\right)^{\delta_{0}} w(x, z)\right|^{2} \mathrm{~d} x \mathrm{~d} z .
\end{aligned}
$$

Since $0<\delta_{0}<\frac{1}{2}$, it follows that $\|\eta w\|_{\mathbf{L}^{2}(\Omega)} \leq C\|\eta\|_{H_{0}^{1}\left(\Gamma_{s}\right)}\|w\|_{\mathbf{L}_{\delta_{0}}^{2}(\Omega)}$.

We denote by $A_{1}^{*} \in \mathcal{L}\left(\mathbf{L}^{2}(\Omega), H^{-2}\left(\Gamma_{s}\right)\right), A_{2}^{*} \in \mathcal{L}\left(\mathbf{L}^{2}(\Omega), L^{2}\left(\Gamma_{s}\right)\right), A_{3}^{*} \in \mathcal{L}\left(\mathbf{L}^{2}(\Omega), H^{-2}\left(\Gamma_{s}\right)\right)$ and $A_{4}^{*} \in \mathcal{L}\left(L^{2}\left(\Gamma_{s}\right), H^{-2}\left(\Gamma_{s}\right)\right)$ the adjoints of $A_{1}, A_{2}, A_{3}$ and $A_{4}$ respectively, where $H^{-2}\left(\Gamma_{s}\right)$ is the dual of $H_{0}^{2}\left(\Gamma_{s}\right)$.

5.2. Definition of the unbounded operator $(\mathcal{A}, D(\mathcal{A}))$. For the beam, we introduce the unbounded operator $\left(A_{\alpha, \beta}, D\left(A_{\alpha, \beta}\right)\right)$ in $H_{0}^{2}\left(\Gamma_{s}\right)$ defined by

$$
D\left(A_{\alpha, \beta}\right)=H^{4}\left(\Gamma_{s}\right) \cap H_{0}^{2}\left(\Gamma_{s}\right) \quad \text { and } \quad A_{\alpha, \beta}=\beta \Delta_{s}-\alpha \Delta_{s}^{2} .
$$

We equip the space $H_{0}^{2}\left(\Gamma_{s}\right)$ with the norm

$$
(\eta, \xi)_{H_{0}^{2}\left(\Gamma_{s}\right)}=\left(\left(-A_{\alpha, \beta}\right)^{\frac{1}{2}} \eta,\left(-A_{\alpha, \beta}\right)^{\frac{1}{2}} \xi\right)_{L^{2}\left(\Gamma_{s}\right)}=\int_{\Gamma_{s}}\left(\beta \nabla_{s} \eta \cdot \nabla_{s} \xi+\alpha \Delta_{s} \eta \Delta_{s} \xi\right)
$$

and the spaces $H=\mathbf{L}^{2}(\Omega) \times H_{0}^{2}\left(\Gamma_{s}\right) \times L^{2}\left(\Gamma_{s}\right)$ and $Z=\mathbf{V}_{n, \Gamma_{0}}^{0}(\Omega) \times H_{0}^{2}\left(\Gamma_{s}\right) \times L^{2}\left(\Gamma_{s}\right)$ with the inner product

$$
\left(\left(v, \eta_{1}, \eta_{2}\right),\left(\phi, \xi_{1}, \xi_{2}\right)\right)_{H}=(v, \phi)_{\mathbf{L}^{2}(\Omega)}+\left(\eta_{1}, \xi_{1}\right)_{H_{0}^{2}\left(\Gamma_{s}\right)}+\left(\eta_{2}, \xi_{2}\right)_{L^{2}\left(\Gamma_{s}\right)} .
$$

As in [24, Lemma 3.2], we can show that $I+\gamma_{s} N_{s}$ is an automorphism in $L^{2}\left(\Gamma_{s}\right)$, and we can introduce $M_{s} \in \mathcal{L}(Z)$ and $M_{s}^{-1} \in \mathcal{L}(Z)$ two automorphisms in $Z$ defined by

$$
M_{s}=\left(\begin{array}{ccc}
I & 0 & 0 \\
0 & I & 0 \\
0 & \gamma_{s} N_{\mathrm{div}} A_{3} & I+\gamma_{s} N_{s}
\end{array}\right) \quad \text { and } \quad M_{s}^{-1}=\left(\begin{array}{ccc}
I & 0 & 0 \\
0 & I & 0 \\
0 & -\left(I+\gamma_{s} N_{s}\right)^{-1} \gamma_{s} N_{\mathrm{div}} A_{3} & \left(I+\gamma_{s} N_{s}\right)^{-1}
\end{array}\right)
$$

REMARK 5.4. We notice that, in $M_{s}, \gamma_{s} N_{s}$ corresponds to the so-called added mass effect (see [5]). Here, the added mass operator $M_{s}$ is not symmetric. 
We are going to define the infinitesimal generator $(\mathcal{A}, D(\mathcal{A}))$ in $Z$ of the linearized system around $(0,0,0,0)$ associated with $(3.3)$. For that we first introduce

$$
D(\mathcal{A}):=\left\{\left(P v, \eta_{1}, \eta_{2}\right) \in \mathbf{V}_{n, \Gamma_{0}}^{\frac{1}{2}+\varepsilon_{0}}(\Omega) \times\left(H^{4}\left(\Gamma_{s}\right) \cap H_{0}^{2}\left(\Gamma_{s}\right)\right) \times H_{0}^{2}\left(\Gamma_{s}\right) \mid P v-P L\left(\eta_{2}, A_{3} \eta_{1}\right) \in D(A)\right\} .
$$

Lemma 5.5. If $\left(P v, \eta_{1}, \eta_{2}\right)$ belongs to $D(\mathcal{A})$, then $N_{v}\left(P v+\nabla N_{s} \eta_{2}+\nabla N_{\text {div }} A_{3} \eta_{1}\right)$ belongs to $H^{\frac{1}{2}+\varepsilon_{0}}(\Omega)$.

Proof. Let $\left(P v, \eta_{1}, \eta_{2}\right)$ belong to $D(\mathcal{A})$. Then, there exists $F \in \mathbf{V}_{n, \Gamma_{0}}^{0}(\Omega)$ such that

$$
\left(\lambda_{0} I-A\right) P v+\left(A-\lambda_{0} I\right) P L\left(\eta_{2}, A_{3} \eta_{1}\right)=F .
$$

We set $v=P v+\nabla N_{s} \eta_{2}+\nabla N_{\text {div }} A_{3} \eta_{1} \quad$ and $\quad \rho=-\lambda_{0} N_{s} \eta_{2}-\lambda_{0} N_{\text {div }} A_{3} \eta_{1}+N_{v} v$. Then, according to Theorem 4.12 , we know that $(v, \rho)$ is the unique solution of the equation

$$
\begin{aligned}
& \lambda_{0} v-\operatorname{div} \sigma(v, \rho)+\left(u_{s} \cdot \nabla\right) v+(v \cdot \nabla) u_{s}=F, \quad \operatorname{div} v=A_{3} \eta_{1} \text { in } \Omega, \\
& v=\eta_{2} n \text { on } \Gamma_{s}, v=0 \text { on } \Gamma_{i}, \quad v \cdot n=0 \text { and } \varepsilon(v) n \cdot \tau=0 \text { on } \Gamma_{e}, \quad \sigma(v, \rho) n=0 \text { on } \Gamma_{n} .
\end{aligned}
$$

According to Lemma 4.9, we conclude that $N_{v}\left(P v+\nabla N_{s} \eta_{2}+\nabla N_{\text {div }} A_{3} \eta_{1}\right)$ belongs to $H^{\frac{1}{2}+\varepsilon_{0}}(\Omega)$.

We are now in position to introduce the unbounded operator $(\mathcal{A}, D(\mathcal{A}))$ in $Z$ where $D(\mathcal{A})$ is defined above and

$$
\mathcal{A}=M_{s}^{-1}\left(\begin{array}{ccc}
A & P A_{1}+\left(\lambda_{0} I-A\right) P L\left(0, A_{3} \cdot\right) & P A_{2}+\left(\lambda_{0} I-A\right) P L(\cdot, 0) \\
0 & 0 & I \\
0 & A_{\alpha, \beta}+\gamma_{s} N_{p} A_{1}+A_{4} & \gamma \Delta+\gamma_{s} N_{p} A_{2}
\end{array}\right)+M_{s}^{-1} \mathcal{A}_{p}
$$

where $\left(\mathcal{A}_{p}, D\left(\mathcal{A}_{p}\right)\right)$ is the unbounded operator in $Z$ defined by $D\left(\mathcal{A}_{p}\right)=D(\mathcal{A})$ and

$$
\mathcal{A}_{p}\left(P v, \eta_{1}, \eta_{2}\right)^{T}=\left(0,0, \gamma_{s} N_{v}\left(P v+\nabla N_{s} \eta_{2}+\nabla N_{\mathrm{div}} A_{3} \eta_{1}\right)\right)^{T}, \quad \forall\left(P v, \eta_{1}, \eta_{2}\right) \in D(\mathcal{A}) .
$$

5.3. Resolvent of $\mathcal{A}$ in $Z$. In what follows, we are going to consider system (5.1) either when $\lambda \in \mathbb{C}$ and $\left(F_{f}, F_{s}^{1}, F_{s}^{2}\right)$ are complex valued functions (in which case the solutions are also complex valued functions), or when $\lambda \in \mathbb{R}$ and $\left(F_{f}, F_{s}^{1}, F_{s}^{2}\right)$ are real valued functions (in which case the solutions are also real valued functions). The context will clearly indicate in which case we shall be.

The goal is to prove that the unbounded operator $(\mathcal{A}, D(\mathcal{A}))$ has a compact resolvent.

Proposition 5.6. Let $\left(F_{f}, F_{s}^{1}, F_{s}^{2}\right)$ belong to $H$ and $\lambda$ belong to $\mathbb{C}$. A quadruplet $\left(v, q, \eta_{1}, \eta_{2}\right) \in H_{\delta_{0}}$ is solution to system (5.1) if and only if

$$
\begin{aligned}
& \lambda\left(P v, \eta_{1}, \eta_{2}\right)^{T}=\mathcal{A}\left(P v, \eta_{1}, \eta_{2}\right)^{T}+M_{s}^{-1}\left(P F_{f}, F_{s}^{1}, F_{s}^{2}+\gamma_{s} N_{p} F_{f}\right)^{T}, \\
& (I-P) v=\nabla N_{s} \eta_{2}+\nabla N_{\mathrm{div}} A_{3} \eta_{1}, \\
& q=-\lambda N_{s} \eta_{2}-\lambda N_{\mathrm{div}} A_{3} \eta_{1}+N_{p} A_{1} \eta_{1}+N_{p} A_{2} \eta_{2}+N_{v}\left(P v+\nabla N_{s} \eta_{2}+\nabla N_{\mathrm{div}} A_{3} \eta_{1}\right)+N_{p} F_{f} .
\end{aligned}
$$

Proof. It follows from Theorem 4.12.

Proposition 5.7. Let $\left(F_{f}, F_{s}^{1}, F_{s}^{2}\right)$ belong to $Z$. If $\lambda \in \mathbb{R}$ is large enough, then system (5.1) admits a unique solution $\left(v, q, \eta_{1}, \eta_{2}\right) \in H_{\delta_{0}}$ satisfying the estimate

$$
\|v\|_{\mathbf{H}_{\delta_{0}}^{2}(\Omega)}+\|q\|_{H_{\delta_{0}}^{1}(\Omega)}+\left\|\eta_{1}\right\|_{H^{4}\left(\Gamma_{s}\right)}+\left\|\eta_{2}\right\|_{H^{2}\left(\Gamma_{s}\right)} \leq C\left\|\left(F_{f}, F_{s}^{1}, F_{s}^{2}\right)\right\|_{Z}
$$

Proof. Step 1. Lifting of the divergence condition. We are going to rewrite system (5.1) in the form of a system with divergence free condition. For that, we make the change of unknowns

$$
\widehat{v}=\left(\widehat{v}_{1}, \widehat{v}_{2}\right)=v-L\left(0, A_{3} \eta_{1}\right) \quad \text { and } \quad \widehat{p}=p-L_{p}\left(0, A_{3} \eta_{1}\right),
$$

where $L$ and $L_{p}$ are introduced in (4.19). We have $\widehat{v}=\eta_{2} n$ on $\Gamma_{s}$, which gives

$$
\eta_{2}=\widehat{v}_{2} \text { on } \Gamma_{s} \quad \text { and } \quad \eta_{1}=\frac{1}{\lambda} \widehat{v}_{2}+\frac{1}{\lambda} F_{s}^{1} \text { on } \Gamma_{s} .
$$


We introduce the operator $L_{\lambda}$ in $H_{0}^{2}\left(\Gamma_{s}\right)$ defined by

$$
L_{\lambda}=\lambda^{2}-(\beta+\gamma \lambda) \Delta_{s}+\alpha \Delta_{s}^{2}-A_{4}-\gamma_{s} L_{p}\left(0, A_{3} \cdot\right) .
$$

We choose $\lambda$ large enough so that $L_{\lambda}$ satisfies a coercivity condition in $H_{0}^{2}\left(\Gamma_{s}\right)$. From Proposition 5.1, it follows that $L_{\lambda} \in \mathcal{L}\left(H_{0}^{2}\left(\Gamma_{s}\right), H^{-2}\left(\Gamma_{s}\right)\right)$, and with the Lax-Milgram Theorem, we can prove that $L_{\lambda}$ is an isomorphism from $H_{0}^{2}\left(\Gamma_{s}\right)$ into $H^{-2}\left(\Gamma_{s}\right)$. The operator $L_{\lambda}$ is also an isomorphism from $H^{4}\left(\Gamma_{s}\right) \cap H_{0}^{2}\left(\Gamma_{s}\right)$ into $L^{2}\left(\Gamma_{s}\right)$. The system satisfied by $\left(\widehat{v}, \widehat{p}, \eta_{1}, \eta_{2}\right)$ can be rewritten in the form

$$
\begin{aligned}
& \lambda \widehat{v}-\operatorname{div} \sigma(\widehat{v}, \widehat{p})+\left(u_{s} \cdot \nabla\right) \widehat{v}+(\widehat{v} \cdot \nabla) u_{s}-A_{5} \widehat{v}=G_{f} \text { in } \Omega, \\
& \operatorname{div} \widehat{v}=0 \text { in } \Omega, \quad \widehat{v}=\lambda L_{\lambda}^{-1} \gamma_{s} \widehat{p} n+G_{b} n \text { on } \Gamma_{s}, \quad \widehat{v}=0 \text { on } \Gamma_{i}, \\
& \widehat{v} \cdot n=0 \text { on } \Gamma_{e}, \quad \varepsilon(\widehat{v}) n \cdot \tau=0 \text { on } \Gamma_{e}, \quad \sigma(\widehat{v}, \widehat{p}) n=0 \text { on } \Gamma_{n}, \\
& \lambda \eta_{1}-\eta_{2}=F_{s}^{1} \text { on } \Gamma_{s}, \quad L_{\lambda} \eta_{1}=\gamma_{s} \widehat{p}+G_{s} \text { on } \Gamma_{s}, \\
& \eta_{1}=0 \text { on } \partial \Gamma_{s}, \quad \eta_{1, x}=0 \text { on } \partial \Gamma_{s},
\end{aligned}
$$

where

$$
\begin{aligned}
& A_{5} \widehat{v}=\frac{1}{\lambda} A_{1} \gamma_{s} \widehat{v}_{2}+A_{2} \gamma_{s} \widehat{v}_{2}+\frac{\lambda_{0}-\lambda}{\lambda} L\left(0, A_{3} \gamma_{s} \widehat{v}_{2}\right)-\frac{1}{\lambda} L_{p}\left(0, A_{3} \gamma_{s} \widehat{v}_{2}\right), \\
& G_{f}=F+\frac{1}{\lambda} A_{1} F_{s}^{1}+\frac{\lambda_{0}-\lambda}{\lambda} L\left(0, A_{3} F_{s}^{1}\right)-\frac{1}{\lambda} L_{p}\left(0, A_{3} F_{s}^{1}\right), \\
& G_{s}=F_{s}^{2}+\lambda F_{s}^{1}-\gamma \Delta F_{s}^{1}, \quad G_{b}=\lambda L_{\lambda}^{-1} G_{s}-F_{s}^{1} .
\end{aligned}
$$

From Theorem 4.1 and Proposition 5.1, it follows that there exists $C>0$ such that

$$
\begin{aligned}
& \left\|A_{5} \widehat{v}\right\|_{\mathbf{L}^{2}(\Omega)} \leq C\left\|\gamma_{s} \widehat{v}_{2}\right\|_{H_{0}^{2}\left(\Gamma_{s}\right)} \quad \text { for all } \widehat{v}_{2} \in H_{0}^{2}\left(\Gamma_{s}\right), \\
& \text { and } \\
& \left\|G_{f}\right\|_{\mathbf{L}^{2}(\Omega)}+\left\|G_{s}\right\|_{L^{2}\left(\Gamma_{s}\right)}+\left\|G_{b}\right\|_{H_{0}^{2}\left(\Gamma_{s}\right)} \leq C\left\|\left(F_{f}, F_{s}^{1}, F_{s}^{2}\right)\right\|_{Z} .
\end{aligned}
$$

Step 2. Existence of weak solutions for system (5.8). We introduce the space

$\mathbf{E}=\left\{\widehat{v}=\left(\widehat{v}_{1}, \widehat{v}_{2}\right) \in \mathbf{H}^{1}(\Omega) \mid \operatorname{div} \widehat{v}=0\right.$ in $\Omega, \widehat{v}=0$ on $\Gamma_{i}, v \cdot n=0$ on $\Gamma_{e}, \widehat{v}_{1}=0$ on $\left.\Gamma_{s},\left.\widehat{v}_{2}\right|_{\Gamma_{s}} \in H_{0}^{2}\left(\Gamma_{s}\right)\right\}$, equipped with the norm $\quad\|\widehat{v}\|_{\mathbf{E}}=\left(\|\widehat{v}\|_{\mathbf{H}^{1}(\Omega)}+\left\|\left.L_{\lambda}^{\frac{1}{2}} \widehat{v}_{2}\right|_{\Gamma_{s}}\right\|_{L^{2}\left(\Gamma_{s}\right)}\right)^{\frac{1}{2}}$. We set $a_{\lambda}(\widehat{v}, \phi)=\lambda \int_{\Omega} \widehat{v} \cdot \phi+2 \nu \int_{\Omega} \varepsilon(\widehat{v}): \varepsilon(\phi)+\int_{\Omega}\left(\left(u_{s} \cdot \nabla\right) \widehat{v}+(\widehat{v} \cdot \nabla) u_{s}\right) \cdot \phi-\int_{\Omega} A_{5} \widehat{v} \cdot \phi+\frac{1}{\lambda} \int_{\Gamma_{s}} L_{\lambda}^{\frac{1}{2}} \widehat{v}_{2} L_{\lambda}^{\frac{1}{2}} \phi_{2}$, and $\quad \ell_{\lambda}(\phi)=\int_{\Omega} G_{f} \cdot \phi+\frac{1}{\lambda} \int_{\Gamma_{s}} L_{\lambda}^{\frac{1}{2}} G_{b} L_{\lambda}^{\frac{1}{2}} \phi_{2}$.

If $(\widehat{v}, \widehat{p}) \in \mathbf{H}_{\delta_{0}}^{2}(\Omega) \times H_{\delta_{0}}^{1}(\Omega)$ is a solution to system (5.8) $)_{1-3}$, then $\widehat{v}$ is solution to the variational problem Find $\widehat{v} \in \mathbf{E}$ such that $a_{\lambda}(\widehat{v}, \phi)=\ell_{\lambda}(\phi), \quad \forall \phi \in \mathbf{E}$.

Thanks to the Lax-Milgram Theorem, problem (5.9) admits a unique solution. Moreover it can be shown that if $\widehat{v} \in \mathbf{E}$ is the solution to (5.9), then there exists a pressure $\widehat{p} \in L^{2}(\Omega)$ such that the pair $(\widehat{v}, \widehat{p})$ is a variational solution to the system

$$
\begin{aligned}
& \lambda_{0} \widehat{v}-\operatorname{div} \sigma(\widehat{v}, \widehat{p})+\left(u_{s} \cdot \nabla\right) \widehat{v}+(\widehat{v} \cdot \nabla) u_{s}=\left(\lambda_{0}-\lambda\right) \widehat{v}+G_{f}-A_{5} \widehat{v}, \quad \operatorname{div} \widehat{v}=0 \text { in } \Omega, \\
& \widehat{v}=\widehat{v}_{2} n \text { on } \Gamma_{s}, \quad \widehat{v}=0 \text { on } \Gamma_{i}, \quad \widehat{v} \cdot n=0 \text { and } \varepsilon(\widehat{v}) n \cdot \tau=0 \text { on } \Gamma_{e}, \quad \sigma(\widehat{v}, \widehat{p}) n=0 \text { on } \Gamma_{n} .
\end{aligned}
$$

By variational solution, we understand a solution to the mixed variational problem associated with (5.10), as introduced in (4.3). Since $\widehat{v}$ belongs to $\mathbf{V}^{1}(\Omega),\left(\lambda_{0}-\lambda\right) \widehat{v}+G_{f}-A_{5} \widehat{v}$ belongs to $\mathbf{L}^{2}(\Omega)$ and $\widehat{v}_{2}$ belongs to $H_{0}^{\frac{3}{2}}\left(\Gamma_{s}\right)$. Thus, from Theorem 4.1, it follows that $(\widehat{v}, \widehat{p})$ belongs to $\mathbf{H}_{\delta_{0}}^{2}(\Omega) \times H_{\delta_{0}}^{1}(\Omega)$ and

$$
\|\widehat{v}\|_{\mathbf{H}_{\delta_{0}}^{2}(\Omega)}+\|\widehat{p}\|_{H_{\delta_{0}}^{1}(\Omega)} \leq C\left(\left\|\left(\lambda_{0}-\lambda\right) \widehat{v}+G_{f}-A_{5} \widehat{v}\right\|_{\mathbf{L}^{2}(\Omega)}+\left\|\widehat{v}_{2}\right\|_{H_{0}^{\frac{3}{2}}\left(\Gamma_{s}\right)}\right) \leq C\left\|\left(F_{f}, F_{s}^{1}, F_{s}^{2}\right)\right\|_{Z} .
$$


Step 3. From equations $(5.8)_{4-6}$, we get

$$
\left\|\eta_{1}\right\|_{H^{4}\left(\Gamma_{s}\right)}+\left\|\eta_{2}\right\|_{H^{2}\left(\Gamma_{s}\right)} \leq C\left\|\left(F_{f}, F_{s}^{1}, F_{s}^{2}\right)\right\|_{Z}
$$

We deduce that system (5.8) admits a unique solution $\left(\widehat{v}, \widehat{p}, \eta_{1}, \eta_{2}\right) \in H_{\delta_{0}}$ satisfying

$$
\|\widehat{v}\|_{\mathbf{H}_{\delta_{0}}^{2}(\Omega)}+\|\widehat{p}\|_{H_{\delta_{0}}^{1}(\Omega)}+\left\|\eta_{1}\right\|_{H^{4}\left(\Gamma_{s}\right)}+\left\|\eta_{2}\right\|_{H^{2}\left(\Gamma_{s}\right)} \leq C\left\|\left(F_{f}, F_{s}^{1}, F_{s}^{2}\right)\right\|_{Z}
$$

Step 4. Existence of solutions for system (5.1). According to Theorem 4.1 and Proposition 5.1, $\left(L\left(0, A_{3} \eta_{1}\right), L_{p}\left(0, A_{3} \eta_{1}\right)\right)$ belongs to $\mathbf{H}_{\delta_{0}}^{2}(\Omega) \times H_{\delta_{0}}^{1}(\Omega)$ and we have

$$
\left\|L\left(0, A_{3} \eta_{1}\right)\right\|_{\mathbf{H}_{\delta_{0}}^{2}(\Omega)}+\left\|L_{p}\left(0, A_{3} \eta_{1}\right)\right\|_{H_{\delta_{0}}^{1}(\Omega)} \leq C\left\|A_{3} \eta_{1}\right\|_{H^{1}(\Omega)} \leq C\left\|\left(F_{f}, F_{s}^{1}, F_{s}^{2}\right)\right\|_{Z}
$$

Thus, thanks to (5.7) and (5.11), system (5.1) admits a unique solution $\left(v, q, \eta_{1}, \eta_{2}\right)$ in $H_{\delta_{0}}$ satisfying

$$
\|v\|_{\mathbf{H}_{\delta_{0}}^{2}(\Omega)}+\|q\|_{H_{\delta_{0}}^{1}(\Omega)}+\left\|\eta_{1}\right\|_{H^{4}\left(\Gamma_{s}\right)}+\left\|\eta_{2}\right\|_{H^{2}\left(\Gamma_{s}\right)} \leq C\left\|\left(F_{f}, F_{s}^{1}, F_{s}^{2}\right)\right\|_{Z}
$$

THEOREM 5.8. The resolvent of the unbounded operator $(\mathcal{A}, D(\mathcal{A}))$ is compact.

Proof. Let $\lambda>0$ large enough, and let $\left(F_{f}, F_{s}^{1}, F_{s}^{2}\right)$ belong to $Z$. There exists $\left(P v, \eta_{1}, \eta_{2}\right) \in Z$ such that

$$
(\lambda I-\mathcal{A})\left(P v, \eta_{1}, \eta_{2}\right)^{T}=M_{s}^{-1}\left(F_{f}, F_{s}^{1}, F_{s}^{2}\right)^{T} .
$$

Thanks to Proposition 5.6, $\left(v, q, \eta_{1}, \eta_{2}\right)$ with

$$
\begin{aligned}
& v=P v+\nabla N_{s} \eta_{2}+\nabla N_{\mathrm{div}} A_{3} \eta_{1} \\
& q=-\lambda N_{s} \eta_{2}-\lambda N_{\mathrm{div}} A_{3} \eta_{1}+N_{p} A_{1} \eta_{1}+N_{p} A_{2} \eta_{2}+N_{v}\left(P v+\nabla N_{s} \eta_{2}+\nabla N_{\mathrm{div}} A_{3} \eta_{1}\right)
\end{aligned}
$$

is solution of system (5.1). Due to Proposition 5.7, $\left(v, q, \eta_{1}, \eta_{2}\right)$ belongs to $H_{\delta_{0}}$ and

$$
\|v\|_{\mathbf{H}_{\delta_{0}}^{2}(\Omega)}+\|q\|_{H_{\delta_{0}}^{1}(\Omega)}+\left\|\eta_{1}\right\|_{H^{4}\left(\Gamma_{s}\right)}+\left\|\eta_{2}\right\|_{H^{2}\left(\Gamma_{s}\right)} \leq C\left\|\left(F_{f}, F_{s}^{1}, F_{s}^{2}\right)\right\|_{Z}
$$

From Lemma 4.3 and Proposition 4.4, it follows that $\left(P v, \eta_{1}, \eta_{2}\right)$ belongs to $\mathbf{H}^{\frac{1}{2}+\varepsilon_{0}}(\Omega) \times\left(H^{4}\left(\Gamma_{s}\right) \cap H_{0}^{2}\left(\Gamma_{s}\right)\right) \times H_{0}^{2}\left(\Gamma_{s}\right)$, and

$$
\|P v\|_{\mathbf{H}^{\frac{1}{2}+\varepsilon_{0}(\Omega)}}+\left\|\eta_{1}\right\|_{H^{4}\left(\Gamma_{s}\right)}+\left\|\eta_{2}\right\|_{H^{2}\left(\Gamma_{s}\right)} \leq C\left\|\left(F_{f}, F_{s}^{1}, F_{s}^{2}\right)\right\|_{Z}
$$

Since the imbedding $\mathbf{H}^{\frac{1}{2}+\varepsilon_{0}}(\Omega) \times\left(H^{4}\left(\Gamma_{s}\right) \cap H_{0}^{2}\left(\Gamma_{s}\right)\right) \times H_{0}^{2}\left(\Gamma_{s}\right) \hookrightarrow Z$ is compact, the proof is complete.

\subsection{Analyticity.}

THEOREM 5.9. The unbounded operator $(\mathcal{A}, D(\mathcal{A}))$ is the infinitesimal generator of an analytic semigroup of class $\mathcal{C}^{0}$ on $Z$.

Proof. First, we introduce the unbounded operator $(\widehat{\mathcal{A}}, D(\widehat{\mathcal{A}}))$ in $Z$ defined by $D(\widehat{\mathcal{A}})=D(\mathcal{A})$ and

$$
\widehat{\mathcal{A}}=\left(\begin{array}{ccc}
A & \left(\lambda_{0} I-A\right) P L\left(0, A_{3} \cdot\right) & \left(\lambda_{0} I-A\right) P L(\cdot, 0) \\
0 & 0 & I \\
0 & A_{\alpha, \beta} & \gamma \Delta
\end{array}\right) .
$$

We set $\quad K_{s}:=\left(I+\gamma_{s} N_{s}\right)^{-1}$. We decompose $\mathcal{A}$ in the form $\mathcal{A}=\widehat{\mathcal{A}}+\widehat{\mathcal{A}}_{1}+\widehat{\mathcal{A}}_{2}+\widehat{\mathcal{A}}_{3}+\mathcal{A}_{p}$, where the operators $\widehat{\mathcal{A}}_{1}, \widehat{\mathcal{A}}_{2}$ and $\widehat{\mathcal{A}}_{3}$ are defined in $D(\widehat{\mathcal{A}})$ by

$$
\begin{aligned}
& \widehat{\mathcal{A}}_{1}\left(P v, \eta_{1}, \eta_{2}\right)^{T}=\left(0,0,\left(K_{s}-I\right) A_{\alpha, \beta} \eta_{1}\right)^{T}, \quad \widehat{\mathcal{A}}_{2}\left(P v, \eta_{1}, \eta_{2}\right)=\left(0,0, \gamma\left(K_{s}-I\right) \Delta \eta_{2}\right)^{T}, \\
& \widehat{\mathcal{A}}_{3}\left(P v, \eta_{1}, \eta_{2}\right)^{T}=\left(P A_{1} \eta_{1}+P A_{2} \eta_{2}, 0, K_{s} \gamma_{s} N_{p} A_{1} \eta_{1}+K_{s} A_{4} \eta_{1}+K_{s} \gamma_{s} N_{p} A_{2} \eta_{2}-K_{s} \gamma_{s} N_{\text {div }} A_{3} \eta_{2}\right)^{T},
\end{aligned}
$$

for all $\left(P v, \eta_{1}, \eta_{2}\right) \in D(\widehat{\mathcal{A}})$, and $\mathcal{A}_{p}$ is given in (5.5). The theorem will be a direct consequence of Theorem 5.10, Propositions 5.11, 5.14 and 5.7. 
THEOREM 5.10. The unbounded operator $(\widehat{\mathcal{A}}, D(\widehat{\mathcal{A}}))$ is the infinitesimal generator of an analytic semigroup of class $\mathcal{C}^{0}$ on $Z$.

Proof. The proof is similar to that in [24, Theorem 3.6].

Proposition 5.11. There exist $0<\theta_{1}, \theta_{2}<1$ such that $\widehat{\mathcal{A}}_{1}$ and $\widehat{\mathcal{A}}_{2}$ belong to $\mathcal{L}\left(D\left((-\widehat{\mathcal{A}})^{\theta_{1}}\right), Z\right)$ and $\mathcal{L}\left(D\left((-\widehat{\mathcal{A}})^{\theta_{2}}\right), Z\right)$ respectively.

Proof. See [24, Lemma 3.9].

Lemma 5.12. There exists $C>0$ such that, for all $\left(P v, \eta_{1}, \eta_{2}\right) \in D(\widehat{\mathcal{A}})$, we have the estimate

$$
\left\|N_{v}\left(P v+\nabla N_{s} \eta_{2}+\nabla N_{\mathrm{div}} A_{3} \eta_{1}\right)\right\|_{H^{\frac{1}{2}+\varepsilon_{0}(\Omega)}} \leq C\left\|\left(P v, \eta_{1}, \eta_{2}\right)\right\|_{D(\widehat{\mathcal{A}})} .
$$

Proof. Let $\lambda$ be positive and $\left(P v, \eta_{1}, \eta_{2}\right)$ be in $D(\widehat{\mathcal{A}})$. We set

$$
\left(F_{f}, F_{s}^{1}, F_{s}^{2}\right)^{T}:=\left(\lambda M_{s}-M_{s} \widehat{\mathcal{A}}\right)\left(P v, \eta_{1}, \eta_{2}\right)^{T} .
$$

As in Proposition 5.6, we can prove that $\left(v, q, \eta_{1}, \eta_{2}\right)$ with

$$
\begin{aligned}
& v=P v+\nabla N_{s} \eta_{2}+\nabla N_{\mathrm{div}} A_{3} \eta_{1}, \\
& q=-\lambda N_{s} \eta_{2}-\lambda N_{\mathrm{div}} A_{3} \eta_{1}+N_{v}\left(P v+\nabla N_{s} \eta_{2}+\nabla N_{\mathrm{div}} A_{3} \eta_{1}\right),
\end{aligned}
$$

is a solution of system (5.1). As in the proof of Proposition 5.7, we can show that, for $\lambda$ large enough, system (5.1) admits a unique solution $\left(v, q, \eta_{1}, \eta_{2}\right) \in H_{\delta_{0}}$ satisfying the estimate

$$
\|v\|_{\mathbf{H}_{\delta_{0}}^{2}(\Omega)}+\|q\|_{H_{\delta_{0}}^{1}(\Omega)}+\left\|\eta_{1}\right\|_{H^{4}\left(\Gamma_{s}\right)}+\left\|\eta_{2}\right\|_{H^{2}\left(\Gamma_{s}\right)} \leq C\left\|\left(F_{f}, F_{s}^{1}, F_{s}^{2}\right)\right\|_{H}
$$

With this estimate and Lemma 4.3, we obtain

$$
\|q\|_{H^{\frac{1}{2}+\varepsilon_{0}(\Omega)}} \leq C\|q\|_{H_{\delta_{0}}^{1}(\Omega)} \leq C\left\|\left(F_{f}, F_{s}^{1}, F_{s}^{2}\right)\right\|_{H} \leq C\left\|\left(P v, \eta_{1}, \eta_{2}\right)\right\|_{D(\widehat{\mathcal{A}})} .
$$

Using Proposition 5.1, we deduce that

$$
\left\|N_{v}\left(P v+\nabla N_{s} \eta_{2}+\nabla N_{\mathrm{div}} A_{3} \eta_{1}\right)\right\|_{H^{\frac{1}{2}+\varepsilon_{0}(\Omega)}} \leq C\left\|\left(P v, \eta_{1}, \eta_{2}\right)\right\|_{D(\widehat{\mathcal{A}})} .
$$

LEMMA 5.13. The operator $K_{s}$ belongs to $\mathcal{L}\left(H^{\varepsilon_{0}}\left(\Gamma_{s}\right)\right)$.

Proof. Let $g$ belong to $H^{\varepsilon_{0}}\left(\Gamma_{s}\right)$. Since $\left.I+\gamma_{s} N_{s}\right) \in \operatorname{isom}\left(L^{2}\left(\Gamma_{s}\right)\right)$, there exists a unique $h \in L^{2}\left(\Gamma_{s}\right)$ such that $h+\gamma_{s} N_{s} h=g$. Since $\gamma_{s} N_{s} h$ belongs to $H^{\varepsilon_{0}}\left(\Gamma_{s}\right)$, we deduce that $h$ belongs to $H^{\varepsilon_{0}}\left(\Gamma_{s}\right)$. Thus, the operator $I+\gamma_{s} N_{s}$ is an automorphism in $H^{\varepsilon_{0}}\left(\Gamma_{s}\right)$.

Proposition 5.14. The operator $\mathcal{A}_{p} \in \mathcal{L}\left(D(\widehat{\mathcal{A}}), L^{2}\left(\Gamma_{s}\right)\right)$ is compact.

Proof. Thanks to Proposition 5.1, Lemmas 5.12 and 5.13, we prove that the operator $\mathcal{A}_{p}$ belongs to $\mathcal{L}\left(D(\widehat{\mathcal{A}}), H^{\varepsilon_{0}}\left(\Gamma_{s}\right)\right)$. From the compact embedding $H^{\varepsilon_{0}}\left(\Gamma_{s}\right) \hookrightarrow L^{2}\left(\Gamma_{s}\right)$, it follows that $\mathcal{A}_{p}$ is a compact operator from $D(\widehat{\mathcal{A}})$ into $L^{2}\left(\Gamma_{s}\right)$.

Proposition 5.15. The operators $\widehat{\mathcal{A}}_{3}$ is $\widehat{\mathcal{A}}$-bounded with relative bound zero.

Proof. It is sufficient to argue by contradiction as in [24, Lemma 3.8].

Proposition 5.16. We have

$$
[Z, D(\mathcal{A})]_{\frac{1}{2}}=\left\{\left(P v, \eta_{1}, \eta_{2}\right) \in \mathbf{V}_{n, \Gamma_{0}}^{\frac{1}{2}+\varepsilon_{0}}(\Omega) \times\left(H^{3} \cap H_{0}^{2}\right)\left(\Gamma_{s}\right) \times H_{0}^{1}\left(\Gamma_{s}\right) \mid P v-P L\left(\eta_{2}, A_{3} \eta_{1}\right) \in \mathbf{V}_{\Gamma_{0}}^{1}(\Omega)\right\} .
$$

Proof. The result can be established by proving that the mapping

$$
\Upsilon:\left(P v, \eta_{1}, \eta_{2}\right) \longmapsto\left(P v-P L\left(\eta_{2}, A_{3} \eta_{1}\right), \eta_{1}, \eta_{2}\right),
$$

is an isomorphism from $D(\mathcal{A})$ into $D(A) \times\left(H^{4}\left(\Gamma_{s}\right) \cap H_{0}^{2}\left(\Gamma_{s}\right)\right) \times H_{0}^{2}\left(\Gamma_{s}\right)$, and from $[Z, D(\mathcal{A})]_{\frac{1}{2}}$ into $\mathbf{V}_{\Gamma_{0}}^{1}(\Omega) \times\left(H^{3}\left(\Gamma_{s}\right) \cap H_{0}^{2}\left(\Gamma_{s}\right)\right) \times H_{0}^{1}\left(\Gamma_{s}\right)$, and by using that $[Z, D(\mathcal{A})]_{\frac{1}{2}} \subset \mathbf{V}_{n, \Gamma_{0}}^{\frac{1}{2}+\varepsilon_{0}}(\Omega) \times\left(H^{3}\left(\Gamma_{s}\right) \cap H_{0}^{2}\left(\Gamma_{s}\right)\right) \times$ $H_{0}^{1}\left(\Gamma_{s}\right)$. 
6. Adjoint of $(\mathcal{A}, D(\mathcal{A}))$. Using (4.9), we introduce the operator $N_{*} \in \mathcal{L}\left(\mathbf{H}_{\delta_{0}}^{2}(\Omega), L^{2}(\Omega)\right)$ defined by $\quad N_{*} \phi=\psi, \quad$ where $\psi$ is the solution of the variational problem

Find $\psi \in L^{2}(\Omega)$ such that

$$
\int_{\Omega} \psi \zeta=2 \nu\left\langle\varepsilon(\phi), \nabla^{2} \chi\right\rangle_{\mathbf{L}_{-\delta_{0}}^{2}(\Omega), \mathbf{L}_{\delta_{0}}^{2}(\Omega)}-2 \nu \int_{\Gamma_{0}} \varepsilon(\phi) n \cdot \nabla \chi+\int_{\Omega}\left[-\left(u_{s} \cdot \nabla\right) \phi+\left(\nabla u_{s}\right)^{T} \phi\right] \cdot \nabla \chi,
$$

for all $\zeta \in L^{2}(\Omega)$, and where $\chi \in H_{\delta_{0}}^{2}(\Omega)$ is the solution of equation (4.8).

We also introduce the lifting operators $D \in \mathcal{L}\left(H_{0}^{\frac{3}{2}}\left(\Gamma_{s}\right), \mathbf{H}_{\delta_{0}}^{2}(\Omega)\right)$ and $D_{p} \in \mathcal{L}\left(H_{0}^{\frac{3}{2}}\left(\Gamma_{s}\right), H_{\delta_{0}}^{1}(\Omega)\right)$ defined by

$$
D g=\phi \quad \text { and } \quad D_{p} g=\psi,
$$

where $(\phi, \psi)$ is the solution of the system

$$
\begin{aligned}
& \lambda_{0} \phi-\operatorname{div} \sigma(\phi, \psi)-\left(u_{s} \cdot \nabla\right) \phi+\left(\nabla u_{s}\right)^{T} \phi=0, \quad \operatorname{div} \phi=0 \text { in } \Omega, \quad \phi=g n \text { on } \Gamma_{s}, \\
& \phi=0 \text { on } \Gamma_{i}, \quad \phi \cdot n=0 \text { and } \varepsilon(\phi) n \cdot \tau=0 \text { on } \Gamma_{e}, \quad \sigma(\phi, \psi) n+u_{s} \cdot n \phi=0 \text { on } \Gamma_{n} .
\end{aligned}
$$

Finally, we introduce the unbounded operator $\left(\mathcal{A}^{\#}, D\left(\mathcal{A}^{\#}\right)\right)$ in $Z$ defined by

$$
\left.D\left(\mathcal{A}^{\#}\right)=\left\{\left(P \phi, \xi_{1}, \xi_{2}\right) \in \mathbf{V}_{n, \Gamma_{0}}^{\frac{1}{2}+\varepsilon_{0}}(\Omega)\right) \times\left(H^{4}\left(\Gamma_{s}\right) \cap H_{0}^{2}\left(\Gamma_{s}\right)\right) \times H_{0}^{2}\left(\Gamma_{s}\right) \mid P\left(\phi-D \xi_{2}\right) \in D\left(A^{*}\right)\right\},
$$

and

$$
\mathcal{A}^{\#}=\left(\begin{array}{ccc}
A^{*} & 0 & \left(\lambda_{0} I-A^{*}\right) P D \\
\left(-A_{\alpha, \beta}\right)^{-1} A_{1}^{*} & 0 & -I+\left(-A_{\alpha, \beta}\right)^{-1}\left(A_{4}^{*}+2 \nu\left(\gamma_{s} A_{3}\right)^{*}+A_{1}^{*} \nabla N_{s}\right) \\
\gamma_{s} N_{*}+A_{2}^{*} & -A_{\alpha, \beta} & \gamma \Delta+\gamma_{s} N_{*} \nabla N_{s}+A_{2}^{*} \nabla N_{s}
\end{array}\right)+\mathcal{A}_{p}^{\#} .
$$

The operator $\mathcal{A}_{p}^{\#}$ is defined in $D\left(\mathcal{A}^{\#}\right)$ by

$$
\mathcal{A}_{p}^{\#}\left(\begin{array}{c}
P \phi \\
\xi_{1} \\
\xi_{2}
\end{array}\right)=\left(\begin{array}{c}
0 \\
-\left(-A_{\alpha, \beta}\right)^{-1} A_{3}^{*} \gamma_{s} N_{*}\left(P \phi+\nabla N_{s} \xi_{1}\right) \\
\gamma_{s} N_{*}\left(P \phi+\nabla N_{s} \xi_{2}\right)
\end{array}\right), \quad \forall\left(P \phi, \xi_{1}, \xi_{2}\right) \in D\left(\mathcal{A}^{\#}\right) .
$$

Lemma 6.1. The adjoint of the operator $M_{s}$ in $Z$ and its inverse are defined by

$$
M_{s}^{*}=\left(\begin{array}{ccc}
I & 0 & 0 \\
0 & I & -\left(-A_{\alpha, \beta}\right)^{-1} A_{3}^{*} N_{s} \\
0 & 0 & I+\gamma_{s} N_{s}
\end{array}\right), \quad\left(M_{s}^{*}\right)^{-1}=\left(\begin{array}{ccc}
I & 0 & 0 \\
0 & I & \left(-A_{\alpha, \beta}\right)^{-1} A_{3}^{*} N_{s}\left(I+\gamma_{s} N_{s}\right)^{-1} \\
0 & 0 & \left(I+\gamma_{s} N_{s}\right)^{-1}
\end{array}\right) .
$$

Proof. The proof follows from the fact that $\left(I+\gamma_{s} N_{s}\right) \in \operatorname{autom}\left(L^{2}\left(\Gamma_{s}\right)\right)$. It is left to the reader. Proposition 6.2. Let $\lambda$ belong to $\mathbb{C}$ and let $\left(G_{f}, G_{s}^{1}, G_{s}^{2}\right)$ belong to $H$. A quadruplet $\left(\phi, \psi, \xi_{1}, \xi_{2}\right) \in$ $H_{\delta_{0}}$ is solution of system

$$
\begin{aligned}
& \lambda \phi-\operatorname{div} \sigma(\phi, \psi)-\left(u_{s} \cdot \nabla\right) \phi+\left(\nabla u_{s}\right)^{T} \phi=G_{f}, \quad \operatorname{div} \phi=0 \text { in } \Omega, \quad \phi=\xi_{2} n \text { on } \Gamma_{s}, \\
& \phi=0 \text { on } \Gamma_{i}, \quad \phi \cdot n=0 \text { and } \varepsilon(\phi) n \cdot \tau=0 \text { on } \Gamma_{e}, \quad \sigma(\phi, \psi) n+u_{s} \cdot n \phi=0 \text { on } \Gamma_{n}, \\
& \lambda \xi_{1}+\xi_{2}-\left(-A_{\alpha, \beta}\right)^{-1}\left(A_{4}^{*}+2 \nu\left(\gamma_{s} A_{3}\right)^{*}\right) \xi_{2}-\left(-A_{\alpha, \beta}\right)^{-1} A_{1}^{*} \phi+\left(-A_{\alpha, \beta}\right)^{-1} A_{3}^{*} \psi=G_{s}^{1} \text { on } \Gamma_{s}, \\
& \lambda \xi_{2}+\beta \Delta_{s} \xi_{1}-\gamma \Delta_{s} \xi_{2}-\alpha \Delta_{s}^{2} \xi_{1}-A_{2}^{*} \phi-\gamma_{s} \psi=G_{s}^{2} \text { on } \Gamma_{s}, \\
& \xi_{1}=0 \text { on } \partial \Gamma_{s}, \quad \xi_{1, x}=0 \text { on } \partial \Gamma_{s},
\end{aligned}
$$

if and only if

$$
\begin{aligned}
& \lambda M_{s}^{*}\left(P \phi, \xi_{1}, \xi_{2}\right)^{T}=\mathcal{A}^{\#}\left(P \phi, \xi_{1}, \xi_{2}\right)^{T}+\left(P G_{f}, G_{s}^{1}, G_{s}^{2}\right)^{T}, \quad(I-P) \phi=\nabla N_{s} \xi_{2}, \\
& \psi=-\lambda N_{s} \xi_{2}+N_{*}\left(\nabla N_{s} \xi_{2}+P \phi\right)+N_{p} G_{f} .
\end{aligned}
$$


Proof. Let $\left(\phi, \psi, \xi_{1}, \xi_{2}\right) \in H_{\delta_{0}}$ be a solution of system (6.2). As in Theorem 4.12, we can prove that $(\phi, \psi)$ is a solution of system

$$
\begin{aligned}
& \left(\lambda I-A^{*}\right) P \phi+\left(A^{*}-\lambda_{0} I\right) P D \xi_{2}=P G_{f}, \quad(I-P) \phi=\nabla N_{s} \xi_{2}, \\
& \psi=-\lambda N_{s} \xi_{2}+N_{*}\left(P \phi+\nabla N_{s} \xi_{2}\right)+N_{p} G_{f} .
\end{aligned}
$$

Replacing the pressure $\psi$ by the above expression in the equations satisfied by $\xi_{1}$ and $\xi_{2}$, we obtain

$$
\begin{aligned}
\lambda \xi_{1}-\lambda\left(-A_{\alpha, \beta}\right)^{-1} A_{3}^{*} N_{s} \xi_{2} & =-\xi_{2}+\left(-A_{\alpha, \beta}\right)^{-1}\left(A_{4}^{*}+2 \nu\left(\gamma_{s} A_{3}\right)^{*}+A_{1}^{*} \nabla N_{s}\right) \xi_{2}+\left(-A_{\alpha, \beta}\right)^{-1} A_{1}^{*} P \phi \\
& -\left(-A_{\alpha, \beta}\right)^{-1} A_{3}^{*} \gamma_{s} N_{*}\left(P \phi+\nabla N_{s} \xi_{1}\right)+G_{s}^{1},
\end{aligned}
$$

and

$$
\lambda\left(I+\gamma_{s} N_{s}\right) \xi_{2}=-A_{\alpha, \beta} \xi_{1}+\gamma \Delta_{s} \xi_{2}+\gamma_{s} N_{*} \nabla N_{s} \xi_{2}+A_{2}^{*} P \phi+\gamma_{s} N_{*}\left(P \phi+\nabla N_{s} \xi_{2}\right)+G_{s}^{2} .
$$

The proof is complete.

Proposition 6.3. The adjoint of $(\mathcal{A}, D(\mathcal{A}))$ in $Z$ is defined by

$$
D\left(\mathcal{A}^{*}\right)=\left\{M_{s}^{*}\left(P \phi, \xi_{1}, \xi_{2}\right) \in Z \mid\left(P \phi, \xi_{1}, \xi_{2}\right) \in D\left(\mathcal{A}^{\#}\right)\right\} \quad \text { and } \quad \mathcal{A}^{*}=\mathcal{A}^{\#} M_{s}^{-*}
$$

Proof. We set $D\left(\widehat{\mathcal{A}}^{*}\right)=\left\{M_{s}^{*}\left(P \phi, \xi_{1}, \xi_{2}\right) \in Z \mid\left(P \phi, \xi_{1}, \xi_{2}\right) \in D\left(\mathcal{A}^{\#}\right)\right\}$, and $\widehat{\mathcal{A}}^{*}=\mathcal{A}^{\#} M_{s}^{-*}$. With a Green's formula, we can prove that the solution $\left(v, q, \eta_{1}, \eta_{2}\right)$ of system $(5.1)$ and the solution $\left(\phi, \psi, \xi_{1}, \xi_{2}\right)$ of system (6.2) satisfy the identity

$$
\left(\left(F_{f}, F_{s}^{1}, F_{s}^{2}\right)^{T},\left(\phi, \xi_{1}, \xi_{2}\right)^{T}\right)_{H}=\left(\left(v, \eta_{1}, \eta_{2}\right)^{T},\left(G_{f}, G_{s}^{1}, G_{s}^{2}\right)^{T}\right)_{H} \cdot
$$

This identity can be interpreted as follows. According to Proposition 5.6, $\left(P v, \eta_{1}, \eta_{1}\right)$ satisfies

$$
\left(\lambda M_{s}-M_{s} \mathcal{A}\right)\left(P v, \eta_{1}, \eta_{2}\right)^{T}=\left(P F_{f}, F_{s}^{1}, F_{s}^{2}\right)^{T} \text {. }
$$

Thanks to Proposition $6.2,\left(P \phi, \xi_{1}, \xi_{2}\right)$ satisfies

$$
\left(\lambda M_{s}^{*}-\mathcal{A}^{\#}\right)\left(P \phi, \xi_{1}, \xi_{2}\right)^{T}=\left(P G_{f}, G_{s}^{1}, G_{s}^{2}\right)^{T}
$$

Thus, identity (6.4) is equivalent to

$$
\left((\lambda I-\mathcal{A})\left(P v, \eta_{1}, \eta_{2}\right)^{T}, M_{s}^{*}\left(P \phi, \xi_{1}, \xi_{2}\right)^{T}\right)_{H}=\left(\left(P v, \eta_{1}, \eta_{2}\right)^{T},\left(\lambda I-\widehat{\mathcal{A}}^{*}\right) M_{s}^{*}\left(P \phi, \xi_{1}, \xi_{2}\right)^{T}\right)_{H} .
$$

From that, we deduce that $D\left(\widehat{\mathcal{A}}^{*}\right) \subset D\left(\mathcal{A}^{*}\right)$. The reverse inclusion can be proved by standard arguments. Therefore, $\left(\widehat{\mathcal{A}}^{*}, D\left(\widehat{\mathcal{A}}^{*}\right)\right)$ is the adjoint of $(\mathcal{A}, D(\mathcal{A}))$ in $Z$, and the proof is complete.

Proposition 6.4. Let $\lambda$ belong to $\mathbb{C}$ and let $\left(G_{f}, G_{s}^{1}, G_{s}^{2}\right)$ belong to $H$. A quadruplet $\left(\phi, \psi, \xi_{1}, \xi_{2}\right) \in$ $H_{\delta_{0}}$ is solution of system (6.2) if and only if

$$
\begin{aligned}
& \lambda M_{s}^{*}\left(\begin{array}{c}
P \phi \\
\xi_{1} \\
\xi_{2}
\end{array}\right)=\mathcal{A}^{*} M_{s}^{*}\left(\begin{array}{c}
P \phi \\
\xi_{1} \\
\xi_{2}
\end{array}\right)+\left(\begin{array}{c}
P G_{f} \\
G_{s}^{1}-\left(-A_{\alpha, \beta}\right)^{-1} A_{3}^{*} \gamma_{s} N_{p} G_{f} \\
G_{s}^{2}+\gamma_{s} N_{p} G_{f}
\end{array}\right), \quad(I-P) \phi=\nabla N_{s} \xi_{2} \\
& \psi=-\lambda N_{s} \xi_{2}+N_{*}\left(P \phi+\nabla N_{s} \xi_{2}\right)+N_{p} G_{f} .
\end{aligned}
$$

Proof. It is a direct consequence of Proposition 6.2 and of definition of $\left(\mathcal{A}^{*}, D\left(\mathcal{A}^{*}\right)\right)$.

7. Eigenvalue problems. The goal of this section is to find relationships between the eigenvalue problem associated to $\mathcal{A}$ and the eigenvalue problem associated to the linearized system associated to (3.3), i.e.

$$
\lambda \in \mathbb{C}, \quad\left(P v, \eta_{1}, \eta_{2}\right) \in D(\mathcal{A}), \quad \lambda\left(P v, \eta_{1}, \eta_{2}\right)^{T}=\mathcal{A}\left(P v, \eta_{1}, \eta_{2}\right)^{T},
$$


and

$$
\begin{aligned}
& \lambda \in \mathbb{C}, \quad\left(v, q, \eta_{1}, \eta_{2}\right) \in H_{\delta_{0}}, \\
& \lambda v-\operatorname{div} \sigma(v, q)+\left(u_{s} \cdot \nabla\right) v+(v \cdot \nabla) u_{s}-A_{1} \eta_{1}-A_{2} \eta_{2}=0, \quad \operatorname{div} v=A_{3} \eta_{1} \text { in } \Omega, \\
& v=\eta_{2} n \text { on } \Gamma_{s}, \quad v=0 \text { on } \Gamma_{i}, \quad v \cdot n=0 \text { and } \varepsilon(v) n \cdot \tau=0 \text { on } \Gamma_{e}, \quad \sigma(v, q) n=0 \text { on } \Gamma_{n}, \\
& \lambda \eta_{1}-\eta_{2}=0 \text { on } \Gamma_{s}, \\
& \lambda \eta_{2}-\beta \Delta_{s} \eta_{1}-\gamma \Delta_{s} \eta_{2}+\alpha \Delta_{s}^{2} \eta_{1}-A_{4} \eta_{1}-\gamma_{s} q=0 \text { on } \Gamma_{s}, \\
& \eta_{1}=0 \text { on } \partial \Gamma_{s}, \quad \eta_{1, x}=0 \text { on } \partial \Gamma_{s} .
\end{aligned}
$$

We also consider both the adjoint eigenvalue problems

$$
\lambda \in \mathbb{C}, \quad\left(P \phi, \xi_{1}, \xi_{2}\right) \in D\left(\mathcal{A}^{*}\right), \quad \lambda\left(P \phi, \xi_{1}, \xi_{2}\right)^{T}=\mathcal{A}^{*}\left(P \phi, \xi_{1}, \xi_{2}\right)^{T},
$$

and

$$
\begin{aligned}
& \lambda \in \mathbb{C}, \quad\left(\phi, \psi, \xi_{1}, \xi_{2}\right) \in H_{\delta_{0}}, \\
& \lambda \phi-\operatorname{div} \sigma(\phi, \psi)-\left(u_{s} \cdot \nabla\right) \phi+\left(\nabla u_{s}\right)^{T} \phi=0, \quad \operatorname{div} \phi=0 \text { in } \Omega, \quad \phi=\xi_{2} n \text { on } \Gamma_{s}, \\
& \phi=0 \text { on } \Gamma_{i}, \quad \phi \cdot n=0 \text { and } \varepsilon(\phi) n \cdot \tau=0 \text { on } \Gamma_{e}, \quad \sigma(\phi, \psi) n+u_{s} \cdot n \phi=0 \text { on } \Gamma_{n}, \\
& \lambda \xi_{1}+\xi_{2}-\left(-A_{\alpha, \beta}\right)^{-1}\left(A_{4}^{*}+2 \nu\left(\gamma_{s} A_{3}\right)^{*}\right) \xi_{2}-\left(-A_{\alpha, \beta}\right)^{-1} A_{1}^{*} \phi+\left(-A_{\alpha, \beta}\right)^{-1} A_{3}^{*} \psi=0 \text { on } \Gamma_{s}, \\
& \lambda \xi_{2}+\beta \Delta_{s} \xi_{1}-\gamma \Delta_{s} \xi_{2}-\alpha \Delta_{s}^{2} \xi_{1}-A_{2}^{*} \phi-\gamma_{s} \psi=0 \text { on } \Gamma_{s}, \\
& \xi_{1}=0 \text { on } \partial \Gamma_{s}, \quad \xi_{1, x}=0 \text { on } \partial \Gamma_{s} .
\end{aligned}
$$

Definition 7.1. A triplet $\left(P v_{k}, \eta_{1, k}, \eta_{2, k}\right) \in D(\mathcal{A})$ is a generalized eigenfunction for problem (7.1) of order $k \geq 1$ associated to a solution $\left(\lambda,\left(P v_{0}, \eta_{1,0}, \eta_{2,0}\right)\right)$ of $\left(\right.$ 7.1) if $\left(P v_{k}, \eta_{1, k}, \eta_{2, k}\right)$ is obtained by solving the chain of equations

$$
(\lambda I-\mathcal{A})\left(P v_{j}, \eta_{1, j}, \eta_{2, j}\right)^{T}=-\left(P v_{j-1}, \eta_{1, j-1}, \eta_{2, j-1}\right)^{T}, \quad \text { for } 1 \leq j \leq k .
$$

A quadruplet $\left(v_{k}, q_{k}, \eta_{1, k}, \eta_{2, k}\right) \in H_{\delta_{0}}$ is a generalized eigenfunction for problem (7.2) of order $k \geq 1$ associated to a solution $\left(\lambda,\left(v_{0}, q_{0}, \eta_{1,0}, \eta_{2,0}\right)\right)$ of (7.2) if $\left(v_{k}, q_{k}, \eta_{1, k}, \eta_{2, k}\right)$ is obtained by solving, for $1 \leq$ $j \leq k$, the chain of systems

$$
\begin{aligned}
& \lambda v_{j}-\operatorname{div} \sigma\left(v_{j}, q_{j}\right)+\left(u_{s} \cdot \nabla\right) v_{j}+\left(v_{j} \cdot \nabla\right) u_{s}-A_{1} \eta_{1, j}-A_{2} \eta_{2, j}=-v_{j-1} \text { in } \Omega, \\
& \operatorname{div} v_{j}=A_{3} \eta_{1, j} \text { in } \Omega, \quad v_{j}=\eta_{2, j} n \text { on } \Gamma_{s}, \quad v_{j}=0 \text { on } \Gamma_{i}, \\
& v_{j} \cdot n=0 \text { on } \Gamma_{e}, \quad \varepsilon\left(v_{j}\right) n \cdot \tau=0 \text { on } \Gamma_{e}, \quad \sigma\left(v_{j}, q_{j}\right) n=0 \text { on } \Gamma_{n}, \\
& \lambda \eta_{1, j}-\eta_{2, j}=-\eta_{1, j-1} \text { on } \Gamma_{s}, \\
& \lambda \eta_{2, j}-\beta \Delta_{s} \eta_{1, j}-\gamma \Delta_{s} \eta_{2, j}+\alpha \Delta_{s}^{2} \eta_{1, j}-A_{4} \eta_{1, j}-\gamma_{s} q_{j}=-\eta_{2, j-1} \text { on } \Gamma_{s}, \\
& \eta_{1, j}=0 \text { on } \partial \Gamma_{s}, \quad \eta_{1, j, x}=0 \text { on } \partial \Gamma_{s} .
\end{aligned}
$$

We have similar statements for the adjoint eigenvalue problems (7.3) and (7.4).

\subsection{Equivalence between direct eigenvalue problems.}

TheOREM 7.2. A couple $\left(\lambda,\left(v, q, \eta_{1}, \eta_{2}\right)\right) \in \mathbb{C} \times H_{\delta_{0}}$ is a solution of eigenvalue problem (7.2) if and only if $\left(\lambda,\left(P v, \eta_{1}, \eta_{2}\right)\right) \in \mathbb{C} \times D(\mathcal{A})$ is a solution of (7.1) and

$$
\begin{aligned}
& (I-P) v=\nabla N_{s} \eta_{2}+\nabla N_{\mathrm{div}} A_{3} \eta_{1}, \\
& q=-\lambda N_{s} \eta_{2}-\lambda N_{\mathrm{div}} A_{3} \eta_{1}+N_{p} A_{1} \eta_{1}+N_{p} A_{2} \eta_{2}+N_{v}\left(P v+\nabla N_{s} \eta_{2}+\nabla N_{\mathrm{div}} A_{3} \eta_{1}\right) .
\end{aligned}
$$

Proof. It follows from Proposition 5.6.

ThEOREM 7.3. A quadruplet $\left(v_{k}, q_{k}, \eta_{1, k}, \eta_{2, k}\right) \in H_{\delta_{0}}$ is a generalized eigenfunction associated with a solution $\left(\lambda,\left(v_{0}, q_{0}, \eta_{1,0}, \eta_{2,0}\right)\right)$ of (7.2) if and only if $\left(P v_{k}, \eta_{1, k}, \eta_{2, k}\right) \in D(\mathcal{A})$ is a generalized eigenfunction for (7.1) associated with a solution $\left(\lambda,\left(P v_{0}, \eta_{1,0}, \eta_{2,0}\right)\right)$ and

$$
\begin{aligned}
& (I-P) v_{k}=\nabla N_{s} \eta_{2, k}+\nabla N_{\mathrm{div}} A_{3} \eta_{1, k} \\
& q_{k}=-\lambda N_{s} \eta_{2, k}-\lambda N_{\mathrm{div}} A_{3} \eta_{1, k}+N_{p} A_{1} \eta_{1, k}+N_{p} A_{2} \eta_{2, k}+N_{v}\left(P v_{k}+\nabla N_{s} \eta_{2, k}+\nabla N_{\mathrm{div}} A_{3} \eta_{1, k}\right) \\
& \quad-N_{s} \eta_{2, k-1}-N_{\operatorname{div}} A_{3} \eta_{1, k-1} .
\end{aligned}
$$


Proof. Thanks to Proposition 5.6, $\left(v_{k}, q_{k}, \eta_{1, k}, \eta_{2, k}\right)$ is a generalized eigenfunction of order $k \geq 1$ associated with a solution $\left(\lambda,\left(v_{0}, q_{0}, \eta_{1,0}, \eta_{2,0}\right)\right)$ of $(7.2)$ if and only if

$$
\begin{aligned}
& \lambda\left(P v_{k}, \eta_{1, k}, \eta_{2, k}\right)^{T}=\mathcal{A}\left(P v_{k}, \eta_{1, k}, \eta_{2, k}\right)-M_{s}^{-1}\left(P v_{k-1}, \eta_{1, k-1}, \eta_{2, k-1}+\gamma_{s} N_{p} v_{k-1}\right)^{T}, \\
& (I-P) v_{k}=\nabla N_{s} \eta_{2, k}+\nabla N_{\mathrm{div}} A_{3} \eta_{1, k}, \\
& \quad q_{k}=-\lambda N_{s} \eta_{2, k}-\lambda N_{\mathrm{div}} A_{3} \eta_{1, k}+N_{p} A_{1} \eta_{1, k}+N_{p} A_{2} \eta_{2, k}+N_{v}\left(P v_{k}+\nabla N_{s} \eta_{2, k}+\nabla N_{\mathrm{div}} A_{3} \eta_{1, k}\right) \\
& \quad-N_{p} v_{k-1},
\end{aligned}
$$

where $\left(v_{k-1}, q_{k-1}, \eta_{1, k-1}, \eta_{2, k-1}\right)$ is a generalized eigenfunction of order $k-1$. Since

$$
N_{p} v_{k-1}=N_{s} \eta_{2, k-1}+N_{\text {div }} A_{3} \eta_{1, k-1}
$$

it follows that

$$
\begin{aligned}
& \lambda\left(P v_{k}, \eta_{1, k}, \eta_{2, k}\right)^{T}=\mathcal{A}\left(P v_{k}, \eta_{1, k}, \eta_{2, k}\right)^{T}-\left(P v_{k-1}, \eta_{1, k-1}, \eta_{2, k-1}\right)^{T} \\
& (I-P) v_{k}=\nabla N_{s} \eta_{2, k}+\nabla N_{\mathrm{div}} A_{3} \eta_{1, k}, \\
& \quad \quad-N_{s} \eta_{2, k-1}-N_{\mathrm{div}} A_{3} \eta_{1, k-1} .
\end{aligned}
$$

We deduce, by induction, that $\left(v_{k}, q_{k}, \eta_{1, k}, \eta_{2, k}\right)$ is a generalized eigenfunction of order $k \geq 1$ associated with a solution $\left(\lambda,\left(v_{0}, q_{0}, \eta_{1,0}, \eta_{2,0}\right)\right)$ of $(7.2)$ if and only if $\left(P v_{k}, \eta_{1, k}, \eta_{2, k}\right)$ is a generalized eigenfunction of order $k \geq 1$ associated with a solution $\left(\lambda,\left(P v_{0}, \eta_{1,0}, \eta_{2,0}\right)\right)$ of $(7.1)$.

\subsection{Equivalence between adjoint eigenvalue problems.}

TheOrem 7.4. A couple $\left(\lambda,\left(\phi, \psi, \xi_{1}, \xi_{2}\right)\right) \in \mathbb{C} \times H_{\delta_{0}}$ is a solution of eigenvalue problem (7.4) if and only if $\left(\lambda, M_{s}^{*}\left(P \phi, \xi_{1}, \xi_{2}\right)\right) \in \mathbb{C} \times D\left(\mathcal{A}^{*}\right)$ is a solution of (7.3) and

$$
(I-P) \phi=\nabla N_{s} \xi_{2}, \quad \psi=-\lambda N_{s} \xi_{2}+N_{*}\left(P \phi+\nabla N_{s} \xi_{2}\right) .
$$

Proof. It follows from Proposition 6.4.

THEOREM 7.5. A quadruplet $\left(\phi_{k}, \psi_{k}, \xi_{1, k}, \xi_{2, k}\right) \in H_{\delta_{0}}$ is a generalized eigenfunction of order $k \geq 1$ associated with a solution $\left(\lambda,\left(\phi_{0}, \psi_{0}, \xi_{1,0}, \xi_{2,0}\right)\right)$ of (7.4) if and only if $M_{s}^{*}\left(P \phi_{k}, \xi_{1, k}, \xi_{2, k}\right) \in D\left(\mathcal{A}^{*}\right)$ is a generalized eigenfunction of order $k \geq 1$ associated with a solution $\left(\lambda,\left(P \phi_{0}, \xi_{1,0}, \xi_{2,0}\right)\right)$ of (7.3) and

$$
(I-P) \phi_{k}=\nabla N_{s} \xi_{2, k}, \quad \psi_{k}=-\lambda N_{s} \xi_{2, k}+N_{*}\left(P \phi_{k}+\nabla N_{s} \xi_{2, k}\right)+N_{s} \xi_{2, k-1} .
$$

Proof. It is similar to that of Theorem 7.3 and relies on Proposition 6.4.

\section{Stabilization of the linearized system.}

8.1. Projected systems. Let $\left(\lambda_{j}\right)_{j \in \mathbb{N}^{*}}$ be the spectrum of $\mathcal{A}$. We denote by $G_{\mathbb{R}}\left(\lambda_{j}\right)$ the real generalized eigenspace of $\mathcal{A}$, that is the space generated by $\operatorname{Re} G_{\mathbb{C}}\left(\lambda_{j}\right) \cup \operatorname{Im} G_{\mathbb{C}}\left(\lambda_{j}\right)$, where $G_{\mathbb{C}}\left(\lambda_{j}\right)$ is the complex generalized eigenspace of $\mathcal{A}$, and $G_{\mathbb{R}}^{*}\left(\lambda_{j}\right)$ is the real generalized eigenspace of $\mathcal{A}^{*}$ associated to the eigenvalue $\lambda_{j}$. Let $\omega>0$ be such that $-\omega \notin\left\{\operatorname{Re} \lambda_{j} \mid j \in \mathbb{N}^{*}\right\}$. We define the unstable subspaces

$$
Z_{u}=\oplus_{j \in J_{u}} G_{\mathbb{R}}\left(\lambda_{j}\right) \quad \text { and } \quad Z_{u}^{*}=\oplus_{j \in J_{u}} G_{\mathbb{R}}^{*}\left(\lambda_{j}\right),
$$

where $J_{u}$ is a finite subset of $\mathbb{N}^{*}$ such that $\operatorname{Re} \lambda_{j}>-\omega$ for all $j \in J_{u}$, and $\operatorname{Re} \lambda_{j}<-\omega$ for all $j \notin J_{u}$. There exist two subspaces $Z_{s}$ and $Z_{s}^{*}$, invariant under $\left(e^{t \mathcal{A}}\right)_{t \geq 0}$ and $\left(e^{t \mathcal{A}^{*}}\right)_{t \geq 0}$, such that

$$
Z=Z_{u} \oplus Z_{s} \quad \text { and } \quad Z=Z_{u}^{*} \oplus Z_{s}^{*} .
$$

We have identified $Z^{*}$ with $Z$. We denote by $\pi_{u}$ the projection from $Z$ onto $Z_{u}$ along $Z_{s}$ and by $\pi_{s}$ the projection from $Z$ onto $Z_{s}$ along $Z_{u}$. We denote by $d_{u}$ the dimension of the subspace $Z_{u}$. We characterize $\pi_{u}$ in Proposition 8.2. 
Lemma 8.1. Let $\left(P v, \eta_{1}, \eta_{2}\right)$ belong to $Z$, and $\left(P \phi, \xi_{1}, \xi_{2}\right)$ belong to $Z^{*}$. Let us set $\widetilde{v}=P v+$ $\nabla N_{s} \eta_{2}+\nabla N_{\text {div }} A_{3} \eta_{1}$ and $\widetilde{\phi}=P \phi+\nabla N_{s} \xi_{2}$. Then $P \widetilde{v}=P v,(I-P) \widetilde{v}=\nabla N_{s} \eta_{2}+\nabla N_{\text {div }} A_{3} \eta_{1}, P \widetilde{\phi}=P \phi$, $(I-P) \widetilde{\phi}=\nabla N_{s} \xi_{2}$, and we have

$$
\left(\left(\widetilde{v}, \eta_{1}, \eta_{2}\right)^{T},\left(\widetilde{\phi}, \xi_{1}, \xi_{2}\right)^{T}\right)_{H}=\left(\left(P v, \eta_{1}, \eta_{2}\right)^{T}, M_{s}^{*}\left(P \phi, \xi_{1}, \xi_{2}\right)\right)_{H}
$$

Proof. It is clear that $P \widetilde{v}=P v,(I-P) \widetilde{v}=\nabla N_{s} \eta_{2}+\nabla N_{\text {div }} A_{3} \eta_{1}, P \widetilde{\phi}=P \phi$, and $(I-P) \widetilde{\phi}=\nabla N_{s} \xi_{2}$. Using the definitions of $\widetilde{v}$ and $\widetilde{\phi}$, we have

$$
(\widetilde{v}, \widetilde{\phi})_{\mathbf{L}^{2}(\Omega)}=(P v, P \phi)_{\mathbf{V}_{n, \Gamma_{0}}^{0}(\Omega)}+\left(\eta_{2}, \gamma_{s} N_{s} \xi_{2}\right)_{L^{2}\left(\Gamma_{s}\right)}+\left(\eta_{1},-\left(-A_{\alpha, \beta}\right)^{-1} A_{3}^{*} N_{s} \xi_{2}\right)_{H_{0}^{2}\left(\Gamma_{s}\right)} .
$$

We obtain $(8.2)$ by adding $\left(\eta_{1}, \xi_{1}\right)_{H_{0}^{2}\left(\Gamma_{s}\right)}+\left(\eta_{2}, \xi_{2}\right)_{L^{2}\left(\Gamma_{s}\right)}$ in both sides of the above identity.

Proposition 8.2. There exist two families $\left(v_{i}, \eta_{1, i}, \eta_{2, i}\right)_{1 \leq i \leq d_{u}}$ and $\left(\phi_{i}, \xi_{1, i}, \xi_{2, i}\right)_{1 \leq i \leq d_{u}}$ of real valued functions satisfying the following statements:

(i) The family $\left(P v_{i}, \eta_{1, i}, \eta_{2, i}\right)_{1 \leq i \leq d_{u}}$ is a basis of $Z_{u}$.

(ii) The family $\left(M_{s}^{*}\left(P \phi_{i}, \xi_{1, i}, \bar{\xi}_{2, i}\right)\right)_{1 \leq i \leq d_{u}}$ is a basis of $Z_{u}^{*}$.

(iii) We have the bi-orthogonality conditions

$$
\left(\left(v_{i}, \eta_{1, i}, \eta_{2, i}\right)^{T},\left(\phi_{j}, \xi_{1, j}, \xi_{2, j}\right)^{T}\right)_{H}=\delta_{i, j} \quad \text { and } \quad\left(\left(P v_{i}, \eta_{1, i}, \eta_{2, i}\right)^{T}, M_{s}^{*}\left(P \phi_{j}, \xi_{1, j}, \xi_{2, j}\right)^{T}\right)_{H}=\delta_{i, j}
$$

for all $1 \leq i, j \leq d_{u}$.

(iv) The projector $\pi_{u}$ is characterized by

$$
\pi_{u}\left(v, \eta_{1}, \eta_{2}\right)^{T}=\sum_{i=1}^{d_{u}}\left(\left(v, \eta_{1}, \eta_{2}\right)^{T}, M_{s}^{*}\left(P \phi_{i}, \xi_{1, i}, \xi_{2, i}\right)^{T}\right)_{H}\left(P v_{i}, \eta_{1, i}, \eta_{2, i}\right)^{T}, \quad \forall\left(v, \eta_{1}, \eta_{2}\right)^{T} \in Z .
$$

(v) The matrix $\Lambda_{u}$ defined by

$$
\Lambda_{u}=\left[\Lambda_{i, j}\right]_{1 \leq i, j \leq d_{u}} \quad \text { with } \quad \Lambda_{i, j}=\left(\mathcal{A}\left(P v_{i}, \eta_{1, i}, \eta_{2, i}\right)^{T}, M_{s}^{*}\left(P \phi_{j}, \xi_{1, j}, \xi_{2, j}\right)^{T}\right)_{H},
$$

is constituted of real Jordan blocks.

(vi) For all $1 \leq i \leq d_{u}$, there exists $q_{i} \in H_{\delta_{0}}^{1}(\Omega)$ such that $\left(v_{i}, q_{i}, \eta_{1, i}, \eta_{2, i}\right) \in H_{\delta_{0}}$ is a real or imaginary part of a generalized eigenfunction of (7.2), and there exists $\psi_{i} \in H_{\delta_{0}}^{1}(\Omega)$ such that $\left(\phi_{i}, \psi_{i}, \xi_{1, i}, \xi_{2, i}\right) \in H_{\delta_{0}}$ is a real or imaginary part of a generalized eigenfunction of (7.4).

Proof. Arguing as in [9, Lemma 6.2] or in [25], since $\mathcal{A}$ is the infinitesimal generator of an analytic semigroup with compact resolvent, there exist a basis $\left(\widetilde{v}_{i}, \eta_{1, i}, \eta_{2, i}\right)_{1 \leq i \leq d_{u}}$ of $Z_{u}$ constituted of real or imaginary parts of eigenfunctions and generalized eigenfunctions of $\mathcal{A}$ and a basis $\left(M_{s}^{*}\left(\widetilde{\phi}_{i}, \xi_{1, i}, \xi_{2, i}\right)\right)_{1 \leq i \leq d_{u}}$ of $Z_{u}^{*}$ constituted of the real or imaginary parts of eigenfunctions and generalized eigenfunctions of $\mathcal{A}^{*}$, such that

$$
\begin{aligned}
& \left(\left(\widetilde{v}_{i}, \eta_{1, i}, \eta_{2, i}\right)^{T}, M_{s}^{*}\left(\widetilde{\phi}_{j}, \xi_{1, j}, \xi_{2, j}\right)^{T}\right)_{H}=\delta_{i, j}, \quad \forall 1 \leq i, j \leq d_{u}, \\
& \pi_{u}\left(v, \eta_{1}, \eta_{2}\right)^{T}=\sum_{i=1}^{d_{u}}\left(\left(v, \eta_{1}, \eta_{2}\right)^{T}, M_{s}^{*}\left(\widetilde{\phi}_{i}, \xi_{1, i}, \xi_{2, i}\right)\right)_{H}\left(\widetilde{v}_{i}, \eta_{1, i}, \eta_{2, i}\right)^{T},
\end{aligned}
$$

and the matrix $\Lambda_{u}$ is constituted of real Jordan blocks.

We set $v_{i}=\widetilde{v}_{i}+\nabla N_{s} \eta_{2, i}+\nabla N_{\operatorname{div}} A_{3} \eta_{1, i}$ and $\phi_{i}=\tilde{\phi}_{i}+\nabla N_{s} \xi_{2, i}$. Thus, we have $P v_{i}=\tilde{v}_{i}, P \phi_{i}=\tilde{\phi}_{i}$, and assertions (i), (ii) and (v) are proved. Assertion (iii) follows from Lemma 8.1.

Assertion (vi) comes from Theorems 7.3 and 7.5.

We introduce the matrix $B_{u}$ defined by

$$
B_{u}=\left[B_{i, j}\right]_{1 \leq i \leq d_{u}, 1 \leq j \leq n_{c}} \quad \text { with } \quad B_{i, j}=\left(w_{i}, \xi_{2, j}\right)_{L^{2}\left(\Gamma_{s}\right)}
$$

We set $\mathcal{A}_{s, \omega}=\pi_{s}(\mathcal{A}+\omega I) \quad$ and $\quad \mathcal{B}_{s}=\pi_{s} \mathcal{B}$. We notice that

$$
\left\|e^{t \mathcal{A}_{s, \omega}}\right\|_{\mathcal{L}(Z)} \leq C e^{-\varepsilon_{s} t} \quad \forall t>0, \quad \text { with } 0<\varepsilon_{s}<\operatorname{dist}\left(\operatorname{Re} \sigma\left(\mathcal{A}_{s, \omega}\right), 0\right) .
$$

As in [21], we shall see that the stabilization of the linearized system is reduced to the stabilization of the pair $\left(\Lambda_{u}+\omega I, B_{u}\right)$. 
8.2. Stabilizability of the pair $\left(\Lambda_{u}+\omega I, B_{u}\right)$. First, we introduce the unbounded operator $\left(A_{s}, D\left(A_{s}\right)\right)$ in $H_{0}^{2}\left(\Gamma_{s}\right) \times L^{2}\left(\Gamma_{s}\right)$ defined by

$$
D\left(A_{s}\right)=\left(H^{4}\left(\Gamma_{s}\right) \cap H_{0}^{2}\left(\Gamma_{s}\right)\right) \times H_{0}^{2}\left(\Gamma_{s}\right) \quad \text { and } \quad A_{s}=\left(\begin{array}{cc}
0 & I \\
A_{\alpha, \beta}+A_{4}+2 \nu \gamma_{s} A_{3} & \gamma \Delta_{s}
\end{array}\right) .
$$

Let $\lambda$ be in $\mathbb{C}$. We introduce the unbounded operator $\left(L_{*, \lambda}, D\left(L_{*, \lambda}\right)\right)$ defined on $L^{2}\left(\Gamma_{s}\right)$ by $D\left(L_{*, \lambda}\right)=$ $H_{0}^{2}\left(\Gamma_{s}\right)$ and

$$
L_{*, \lambda}=I+\left(-A_{\alpha, \beta}\right)^{-1}\left(\lambda^{2}-\gamma \lambda \Delta_{s}-A_{4}^{*}-2 \nu\left(\gamma_{s} A_{3}\right)^{*}\right) .
$$

Lemma 8.3. If $\lambda$ does not belong to $\operatorname{spect}\left(A_{s}\right)$, then $L_{*, \lambda}$ is an isomorphism from $H^{4}\left(\Gamma_{s}\right) \cap H_{0}^{2}\left(\Gamma_{s}\right)$ into $L^{2}\left(\Gamma_{s}\right)$ and we have

$$
\left(\lambda I-A_{s}^{*}\right)^{-1}=\left(\begin{array}{cc}
\left(-A_{\alpha, \beta}\right)^{-1}(\lambda-\gamma \Delta) L_{*, \lambda}^{-1} & \left(-A_{\alpha, \beta}\right)^{-1}\left(\lambda^{2}-\gamma \lambda \Delta\right) L_{*, \lambda}^{-1}\left(-A_{\alpha, \beta}\right)^{-1}-\left(-A_{\alpha, \beta}\right)^{-1} \\
L_{*, \lambda}^{-1} & \lambda L_{*, \lambda}^{-1}\left(-A_{\alpha, \beta}\right)^{-1}
\end{array}\right) .
$$

For all $j \in J_{u}\left(J_{u}\right.$ appears in (8.1)), we introduce the space $E\left(\lambda_{j}\right)$ defined by

$$
E\left(\lambda_{j}\right)=\left\{\left(\phi, \psi, \xi_{1}, \xi_{2}\right) \in H_{\delta_{0}} \mid\left(\lambda_{j},\left(\phi, \psi, \xi_{1}, \xi_{2}\right)\right) \text { is solution to the eigenvalue problem (7.4) }\right\} .
$$

We choose the family $\left(w_{i}\right)_{1 \leq i \leq n_{c}}$ such that

$$
\operatorname{Vect}\left\{w_{i} \mid 1 \leq i \leq n_{c}\right\}=\operatorname{Vect}\left\{\operatorname{Re} \xi_{2, j}^{k}, \operatorname{Im} \xi_{2, j}^{k} \mid j \in J_{u}, 1 \leq k \leq d_{j}\right\},
$$

where $\left(\phi_{j}^{k}, \psi_{j}^{k}, \xi_{1, j}^{k}, \xi_{2, j}^{k}\right)_{1 \leq i \leq d_{j}}$ is a basis of $E\left(\lambda_{j}\right)$ and "Vect" stands for the vector space spanned by the family within the brackets. The number $d_{j}$ is the dimension of $E\left(\lambda_{j}\right)$. It also corresponds to the geometrical multiplicity of the eigenvalue $\lambda_{j}$ of $A$.

Assumption 2. We assume that $-\omega \notin \operatorname{spect}(\mathcal{A}), 0 \notin \operatorname{spect}(A)$ and

$$
\{\lambda \in \operatorname{spect}(A) \mid \operatorname{Re} \lambda \geq-\omega\} \cap\left\{\lambda \in \operatorname{spect}\left(A_{s}\right) \mid \operatorname{Re} \lambda \geq-\omega\right\}=\emptyset .
$$

We consider the following eigenvalue problem

$$
\begin{aligned}
& \lambda \in \mathbb{C}^{*}, \quad \operatorname{Re} \lambda \geq-\omega, \\
& \lambda \phi-\operatorname{div} \sigma(\phi, \psi)-\left(u_{s} \cdot \nabla\right) \phi+\left(\nabla u_{s}\right)^{T} \phi=0 \text { in } \Omega, \quad \operatorname{div} \phi=0 \text { in } \Omega, \quad \phi=0 \text { on } \Gamma_{s} .
\end{aligned}
$$

Assumption 3. All solution $(\lambda, \phi, \psi)$ to (8.6) obeys the following unique continuation property:

$$
\text { If } \lambda\left(A_{2}^{*} \phi+\gamma_{s} \psi\right)=A_{3}^{*} \psi-A_{1}^{*} \phi, \quad \text { then } \quad(\phi, \psi)=(0,0) .
$$

REMARK 8.4. When $u_{s}=0$ (i.e. in the case of the Stokes system), the unique continuation property stated in (8.7) is proved in [22] and [23]. Unfortunately, the proofs given in these two papers cannot be adapted to the case $u_{s} \neq 0$. Indeed, a necessary and may be not sufficient condition to adapt those proofs should be to assume that $u_{s}$ is analytic. However, this property can be verified numerically, see [8].

THEOREM 8.5. We assume that Assumptions 1, 2, and 3 are satisfied, and that $\left(w_{i}\right)_{1 \leq i \leq n_{c}}$ is given by (8.4). Then, the pair $\left(\Lambda_{u}+\omega I_{\mathbb{R}^{n_{c}}}, B_{u}\right)$ is stabilizable.

Proof. Thanks to [4, Part III, Chapter 1, Proposition 3.3], the pair $\left(\Lambda_{u}+\omega I_{\mathbb{R}^{n_{c}}}, B_{u}\right)$ is stabilizable if and only if

$$
\operatorname{Ker}\left(\lambda I-\mathcal{A}^{*}\right) \cap \operatorname{Ker}\left(\mathcal{B}^{*}\right)=\{0\} \text { for all } \lambda \in \mathbb{C} \text { such that } \operatorname{Re} \lambda \geq-\omega .
$$

Let $M_{s}^{*}\left(P \phi, \xi_{1}, \xi_{2}\right)$ belong to $\operatorname{Ker}\left(\lambda I-\mathcal{A}^{*}\right) \cap \operatorname{Ker}\left(\mathcal{B}^{*}\right)$. We set $(I-P) \phi=\nabla N_{s} \xi_{2}$. From Proposition 6.4, it follows that there exists $\psi \in H_{\delta_{0}}^{1}(\Omega)$ such that $\left(\lambda,\left(\phi, \psi, \xi_{1}, \xi_{2}\right)\right) \in \mathbb{C} \times H_{\delta_{0}}$ is solution to the eigenvalue problem (7.4). Since $\mathcal{B}^{*} M_{s}^{*}\left(P \phi, \xi_{1}, \xi_{2}\right)^{T}=0$, we have

$$
\left(\int_{\Gamma_{s}} w_{i} \cdot \xi_{2}\right)_{1 \leq i \leq n_{c}}=0 .
$$


We are going to prove that $\left(\phi, \psi, \xi_{1}, \xi_{2}\right)=(0,0,0,0)$. From equation (8.8) and due to the construction of $\left(w_{i}\right)_{1 \leq i \leq n_{c}}$, it follows that $\xi_{2}=0$. Next, we can distinguish two cases.

Case 1. $\lambda \notin \operatorname{spect}(A)$. Then, we have $(\phi, \psi)=(0,0)$ and, by using equation $(7.4)_{4}$, we also have $\xi_{1}=0$. Case 2. $\lambda \in \operatorname{spect}(A)$. In that case, $\lambda$ does not belong to $\operatorname{spect}\left(A_{s}\right)$. Therefore, we have

$$
\left.\left(\xi_{1}, \xi_{2}\right)^{T}=\left(\lambda I-A_{s}^{*}\right)^{-1}\left(-A_{\alpha, \beta}\right)^{-1}\left(A_{1}^{*} \phi-A_{3}^{*} \psi\right), A_{2}^{*} \phi+\gamma_{s} \psi\right)^{T},
$$

which implies that $\xi_{2}=L_{*, \lambda}^{-1}\left(-A_{\alpha, \beta}\right)^{-1}\left(A_{1}^{*} \phi-A_{3}^{*} \psi+\lambda A_{2}^{*} \phi+\lambda \gamma_{s} \psi\right)$. Since $\xi_{2}=0$, we have $\lambda\left(A_{2}^{*} \phi+\gamma_{s} \psi\right)=$ $A_{3}^{*} \psi-A_{1}^{*} \phi$. Thus, thanks to Assumption 3, we have $(\phi, \psi)=(0,0)$. Equation $(7.4)_{4}$ gives $\xi_{1}=0$.

8.3. The feedback law. We are going to determine a feedback law of finite dimension able to stabilize the linearized system associated to (3.3). For that, it is sufficient to find a feedback law stabilizing the pair $\left(\Lambda_{u}+\omega I, B_{u}\right)$. Since $\left(\Lambda_{u}+\omega I, B_{u}\right)$ is stabilizable and $-\Lambda_{u}-\omega I$ is stable, the following Algebraic Riccati Equation

$$
P_{u} \in \mathbb{R}^{d_{u} \times d_{u}}, \quad P_{u}=P_{u}^{T}>0, \quad P_{u}\left(\Lambda_{u}+\omega I_{\mathbb{R}^{d_{u}}}\right)+\left(\Lambda_{u}^{T}+\omega I_{\mathbb{R}^{d_{u}}}\right) P_{u}-P_{u} B_{u} B_{u}^{T} P_{u}=0,
$$

admits a unique solution $P_{u}$. Moreover, the operator $K_{u}=\left[K_{u}^{i, j}\right]_{1 \leq i \leq n_{c}, 1 \leq j \leq d_{u}}$ defined by $K_{u}=-B_{u}^{T} P_{u}$ provides a stabilizing feedback law for $\left(\Lambda_{u}+\omega I_{\mathbb{R}^{d_{u}}}, B_{u}\right)$. We introduce the operator $\mathcal{K}_{0} \in \mathcal{L}\left(Z, \mathbb{R}^{n_{c}}\right)$ defined by

$$
\mathcal{K}_{0}\left(P v, \eta_{1}, \eta_{2}\right)^{T}=\left(\sum_{j=1}^{d_{u}} K_{u}^{i, j}\left(\left(P v, \eta_{1}, \eta_{2}\right)^{T},\left(P \phi_{j}, \xi_{1, j}, \xi_{2, j}\right)^{T}\right)_{H}\right)_{1 \leq i \leq n_{c}} .
$$

TheOREm 8.6. We assume that Assumptions 1, 2, and 3 are satisfied. Then, the operator $\mathcal{K}_{0}$ provides a stabilizing feedback for $(\mathcal{A}+\omega I, \mathcal{B})$. Moreover, the operator $\mathcal{A}+\omega I+\mathcal{B K}_{0}$, with domain $D\left(\mathcal{A}+\omega I+\mathcal{B K}_{0}\right)=D(\mathcal{A})$, is the infinitesimal generator of an exponentially stable analytic semigroup on $Z$.

Proof. The proof is similar to that of [14, Theorem 3].

Proposition 8.7. Let $\left(P v, \eta_{1}, \eta_{2}\right)$ belong to $Z$. If we choose $(I-P) v=\nabla N_{s} \eta_{2}+\nabla N_{\text {div }} A_{3} \eta_{1} \in$ $\mathbf{L}^{2}(\Omega)$, then we have

$$
\mathcal{K}_{0}\left(P v, \eta_{1}, \eta_{2}\right)^{T}=\mathcal{K}\left(P v+(I-P) v, \eta_{1}, \eta_{2}\right)^{T},
$$

where the operator $\mathcal{K} \in \mathcal{L}\left(H, \mathbb{R}^{n_{c}}\right)$ is defined by

$$
\mathcal{K}\left(v, \eta_{1}, \eta_{2}\right)^{T}=\left(\sum_{j=1}^{d_{u}} K_{u}^{i, j}\left(\left(v, \eta_{1}, \eta_{2}\right)^{T},\left(\phi_{j}, \xi_{1, j}, \xi_{2, j}\right)^{T}\right)_{H}\right)_{1 \leq i \leq n_{c}}, \quad \forall\left(v, \eta_{1}, \eta_{2}\right) \in H .
$$

Proof. It is a consequence of Lemma 8.1.

9. Stabilization of the non-homogeneous linearized system. We are going to prove that $\mathcal{K}$, defined in (8.7), stabilizes the following non-homogeneous closed-loop system

$$
\begin{aligned}
& v_{t}-\operatorname{div} \sigma(v, q)+\left(u_{s} \cdot \nabla\right) v+(v \cdot \nabla) u_{s}-A_{1} \eta_{1}-A_{2} \eta_{2}-\omega v=F_{f} \text { in } Q^{\infty}, \\
& \operatorname{div} v=A_{3} \eta_{1}+\operatorname{div} F_{\operatorname{div}} \text { in } Q^{\infty}, \quad v=\eta_{2} n \text { on } \Sigma_{s}^{\infty}, \quad v=g_{p} \text { on } \Sigma_{i}^{\infty}, \\
& v \cdot n=0 \text { on } \Sigma_{e}^{\infty}, \quad \varepsilon(v) n \cdot \tau=0 \text { on } \Sigma_{e}^{\infty}, \quad \sigma(v, q) n=0 \text { on } \Sigma_{n}^{\infty}, \\
& \eta_{1, t}-\eta_{2}-\omega \eta_{1}=0 \text { on } \Sigma_{s}^{\infty}, \\
& \eta_{2, t}-\beta \Delta_{s} \eta_{1}-\gamma \Delta_{s} \eta_{2}+\alpha \Delta_{s}^{2} \eta_{1}-\omega \eta_{2}=\gamma_{s} q+F_{s}+\sum_{i=1}^{n_{c}}\left[\mathcal{K}\left(v, \eta_{1}, \eta_{2}\right)^{T}\right]_{i} w_{i} \text { on } \Sigma_{s}^{\infty}, \\
& \eta_{1}=0 \text { on }(0, \infty) \times \partial \Gamma_{s}, \quad \eta_{1, x}=0 \text { on }(0, \infty) \times \partial \Gamma_{s}, \\
& v(0)=v^{0} \text { in } \Omega, \quad \eta_{1}(0)=0 \text { on } \Gamma_{s}, \quad \eta_{2}(0)=\eta_{2}^{0} \text { on } \Gamma_{s},
\end{aligned}
$$

where $\left[\mathcal{K}\left(v, \eta_{1}, \eta_{2}\right)^{T}\right]_{i}$ is the $i$-th component of the vector $\mathcal{K}\left(v, \eta_{1}, \eta_{2}\right)^{T} \in \mathbb{R}^{n_{c}}$.

REMARK 9.1. The feedback law $\mathcal{K}$ does not depend on the Leray projector $P$. 
Theorem 9.2. We assume that Assumptions 1, 2, and 3 are satisfied. Let $\left(F_{f}, F_{\mathrm{div}}, F_{s}\right)$ belong to $Y, g_{p}$ belong to $H_{0}^{1}\left(0, \infty ; \mathbf{H}\left(\Gamma_{i}\right)\right)$ and $\left(v^{0}, \eta_{2}^{0}\right)$ belong to $\mathbf{V}_{\Gamma_{i, e}}^{1}(\Omega) \times H_{0}^{1}\left(\Gamma_{s}\right)$ such that $\left.v^{0}\right|_{\Gamma_{s}}=\eta_{2}^{0} n$. We assume that $F_{\mathrm{div}}$ satisfies the following boundary conditions

$$
\begin{aligned}
& F_{\mathrm{div}}=0 \text { on } \Sigma_{s}^{\infty}, \quad F_{\mathrm{div}}=0 \text { on } \Sigma_{i}^{\infty}, \quad F_{\mathrm{div}} \cdot n=0 \text { on } \Sigma_{e}^{\infty}, \\
& \varepsilon\left(F_{\mathrm{div}}\right) n \cdot \tau=0 \text { on } \Sigma_{e}^{\infty}, \quad \varepsilon\left(F_{\mathrm{div}}\right) n=0 \text { on } \Sigma_{n}^{\infty} .
\end{aligned}
$$

The solution $\left(v, q, \eta_{1}, \eta_{2}\right)$ of system (9.1) satisfies the estimate

$$
\left\|\left(v, q, \eta_{1}, \eta_{2}\right)\right\|_{X_{\delta_{0}}} \leq C\left\|\left(v^{0}, \eta_{2}^{0}, g_{p}, F_{f}, F_{\text {div }}, F_{s}\right)\right\| \|
$$

where $\quad\left\|\left(v^{0}, \eta_{2}^{0}, g_{p}, F_{f}, F_{\mathrm{div}}, F_{s}\right)\right\|:=\left\|v^{0}\right\|_{\mathbf{H}^{1}(\Omega)}+\left\|\eta_{2}^{0}\right\|_{H^{1}\left(\Gamma_{s}\right)}+\left\|g_{p}\right\|_{H_{0}^{1}\left(0, \infty ; \mathbf{H}\left(\Gamma_{i}\right)\right)}+\left\|\left(F_{f}, F_{\mathrm{div}}, F_{s}\right)\right\|_{Y}$.

Proof. Step 1. Reformulation of the system. Due to Remark 4.2, the solution $(w, \pi)$ of the system

$$
\begin{aligned}
& \lambda_{0} w-\operatorname{div} \sigma(w, \pi)+\left(u_{s} \cdot \nabla\right) w+(w \cdot \nabla) u_{s}=0, \quad \operatorname{div} w=0 \text { in } \Omega, \\
& w=0 \text { on } \Gamma_{s}, \quad w=g_{p} \text { on } \Gamma_{i}, \quad w \cdot n=0 \text { and } \varepsilon(w) n \cdot \tau=0 \text { on } \Gamma_{e}, \quad \sigma(w, \pi) n=0 \text { on } \Gamma_{n} .
\end{aligned}
$$

satisfies

$$
\|w\|_{H_{0}^{1}\left(0, \infty ; H_{\delta_{0}}^{2}(\Omega)\right)}+\|\pi\|_{L^{2}\left(0, \infty ; H_{\delta_{0}}^{1}(\Omega)\right)} \leq C\left\|g_{p}\right\|_{H_{0}^{1}\left(0, \infty ; \mathbf{H}\left(\Gamma_{i}\right)\right)} .
$$

We set $\widehat{v}=v-F_{\mathrm{div}}-w$ and $\quad \widehat{q}=q-\pi$. The quadruplet $\left(\widehat{v}, \widehat{q}, \eta_{1}, \eta_{2}\right)$ is solution to the system

$$
\begin{aligned}
& \widehat{v}_{t}-\operatorname{div} \sigma(\widehat{v}, \widehat{q})+\left(u_{s} \cdot \nabla\right) \widehat{v}+(\widehat{v} \cdot \nabla) u_{s}-A_{1} \eta_{1}-A_{2} \eta_{2}-\omega \widehat{v}=\widetilde{F}_{f} \text { in } Q^{\infty}, \\
& \operatorname{div} \widehat{v}=A_{3} \eta_{1} \text { in } Q^{\infty}, \quad \widehat{v}=\eta_{2} n \text { on } \Sigma_{s}^{\infty}, \quad \widehat{v}=0 \text { on } \Sigma_{i}^{\infty}, \\
& \widehat{v} \cdot n=0 \text { on } \Sigma_{e}^{\infty}, \quad \varepsilon(\widehat{v}) n \cdot \tau=0 \text { on } \Sigma_{e}^{\infty}, \quad \sigma(\widehat{v}, \widehat{q}) n=0 \text { on } \Sigma_{n}^{\infty}, \\
& \eta_{1, t}-\eta_{2}-\omega \eta_{1}=0 \text { on } \Sigma_{s}^{\infty}, \\
& \eta_{2, t}-\beta \Delta_{s} \eta_{1}-\gamma \Delta_{s} \eta_{2}+\alpha \Delta_{s}^{2} \eta_{1}-\omega \eta_{2}=\gamma_{s} \widehat{q}+\widetilde{F}_{s}+\sum_{i=1}^{n_{c}}\left[\mathcal{K}\left(\widehat{v}, \eta_{1}, \eta_{2}\right)^{T}\right]_{i} w_{i} \text { on } \Sigma_{s}^{\infty}, \\
& \eta_{1}=0 \text { on }(0, \infty) \times \partial \Gamma_{s}, \quad \eta_{1, x}=0 \text { on }(0, \infty) \times \partial \Gamma_{s}, \\
& \widehat{v}(0)=v^{0} \text { in } \Omega, \quad \eta_{1}(0)=0 \text { on } \Gamma_{s}, \quad \eta_{2}(0)=\eta_{2}^{0} \text { on } \Gamma_{s},
\end{aligned}
$$

where

$$
\begin{aligned}
& \widetilde{F}_{f}=F_{f}-F_{\mathrm{div}, t}+2 \nu \operatorname{div} \varepsilon\left(F_{\mathrm{div}}\right)-\left(u_{s} \cdot \nabla\right) F_{\mathrm{div}}-\left(F_{\mathrm{div}} \cdot \nabla\right) u_{s}+\omega F_{\mathrm{div}}+\lambda_{0} w+w_{t}, \\
& \text { and } \widetilde{F}_{s}=F_{s}+\sum_{i=1}^{n_{c}}\left[\mathcal{K}\left(F_{\mathrm{div}}+w, 0,0\right)^{T}\right]_{i} w_{i}+\gamma_{s} \pi .
\end{aligned}
$$

We can verify that $\left(\widehat{v}, \eta_{1}, \eta_{2}\right)=\left(P \widehat{v}, \eta_{1}, \eta_{2}\right)+((I-P) \widehat{v}, 0,0)$ is solution to the system

$$
\begin{aligned}
& \frac{d}{d t}\left(P \widehat{v}, \eta_{1}, \eta_{2}\right)^{T}=\left(\mathcal{A}+\omega I+\mathcal{B} \mathcal{K}_{0}\right)\left(P \widehat{v}, \eta_{1}, \eta_{2}\right)^{T}+\left(P \widetilde{F}_{f}, 0, \widetilde{F}_{s}+\gamma_{s} N \widetilde{F}_{f}\right), \\
& \left(P \widehat{v}, \eta_{1}, \eta_{2}\right)^{T}(0)=\left(P v^{0}, 0, \eta_{2}^{0}\right)^{T}, \quad(I-P) \widehat{v}=\nabla N_{s} \eta_{2}+\nabla N_{\text {div }} A_{3} \eta_{1} .
\end{aligned}
$$

This is the analogue of Proposition 5.6 for time dependent problems.

Step 2. Regularity of solutions to system (9.5). Thanks to Propositions 5.16 and 5.1 , we prove that $\left(P v^{0}, 0, \eta_{2}^{0}\right)$ belongs to $[D(\mathcal{A}), Z]_{\frac{1}{2}},\left(P \widetilde{F}_{f}, 0, F_{s}+\gamma_{s} N_{p} \widetilde{F}_{f}\right)$ belongs to $L^{2}(0, \infty ; Z)$ and we have the estimate

$$
\left\|\left(P v^{0}, 0, \eta_{2}^{0}\right)\right\|_{[D(\mathcal{A}), Z]_{\frac{1}{2}}}+\left\|\left(P \widetilde{F}_{f}, 0, F_{s}+\gamma_{s} N_{p} \widetilde{F}_{f}\right)\right\|_{L^{2}(0, \infty ; Z)} \leq C\left\|\left(v^{0}, \eta_{2}^{0}, g_{p}, F_{f}, F_{\mathrm{div}}, F_{s}\right)\right\| .
$$

Since $\left(\mathcal{A}+\omega I+\mathcal{B K}_{0}, D\left(\mathcal{A}+\omega I+\mathcal{B K}_{0}\right)\right)$ is the infinitesimal generator of an exponentially stable analytic semigroup on $Z$, it follows that

$$
\left\|\left(P \widehat{v}, \eta_{1}, \eta_{2}\right)\right\|_{L^{2}(0, \infty ; D(\mathcal{A})) \cap H^{1}(0, \infty ; Z)} \leq C\left\|\left(v^{0}, \eta_{2}^{0}, g_{p}, F_{f}, F_{\mathrm{div}}, F_{s}\right)\right\|,
$$

(see [3, Chapter 1, Theorem 3.1]). In particular, we have

$$
\|P \widehat{v}\|_{H^{1}\left(0, \infty ; \mathbf{L}^{2}(\Omega)\right)}+\left\|\eta_{1}\right\|_{H^{4,2}\left(\Sigma_{s}^{\infty}\right)}+\left\|\eta_{2}\right\|_{H^{2,1}\left(\Sigma_{s}^{\infty}\right)} \leq C\left\|\left(v^{0}, \eta_{2}^{0}, g_{p}, F_{f}, F_{\mathrm{div}}, F_{s}\right)\right\| .
$$


From (9.6) and (9.8), it follows that $(I-P) \widehat{v}$ belongs to $H^{1}\left(0, \infty ; \mathbf{L}^{2}(\Omega)\right)$. It implies that $\widehat{v}=P \widehat{v}+(I-$ $P) \widehat{v} \in H^{1}\left(0, \infty ; L^{2}(\Omega)\right)$ and

$$
\|\widehat{v}\|_{H^{1}\left(0, \infty ; \mathbf{L}^{2}(\Omega)\right)} \leq C\left\|\left(v^{0}, \eta_{2}^{0}, g_{p}, F_{f}, F_{\text {div }}, F_{s}\right)\right\| .
$$

For all $t>0,(\widehat{v}(t), \widehat{q}(t))$ is solution to the system

$$
\begin{aligned}
& \lambda_{0} \widehat{v}(t)-\operatorname{div} \sigma(\widehat{v}(t), \widehat{q}(t))+\left(u_{s} \cdot \nabla\right) \widehat{v}(t)+(\widehat{v}(t) \cdot \nabla) u_{s}=G(t) \text { in } \Omega, \\
& \operatorname{div} \widehat{v}(t)=A_{3} \eta_{1}(t) \text { in } \Omega, \quad \widehat{v}(t)=\eta_{2}(t) n \text { on } \Gamma_{s}, \quad \widehat{v}(t)=0 \text { on } \Gamma_{i}, \\
& \widehat{v}(t) \cdot n=0 \text { on } \Gamma_{e}, \quad \varepsilon(\widehat{v}(t)) n \cdot \tau=0 \text { on } \Gamma_{e}, \quad \sigma(\widehat{v}(t), \widehat{q}(t)) n=0 \text { on } \Gamma_{n},
\end{aligned}
$$

where

$$
G=-\widehat{v}_{t}+\left(\lambda_{0}+\omega\right) \widehat{v}-\left(u_{s} \cdot \nabla\right) \widehat{v}-(\widehat{v} \cdot \nabla) u_{s}+A_{1} \eta_{1}+A_{2} \eta_{2}+\widetilde{F}_{f} .
$$

From (9.7), (9.8) and (9.9), it follows that $G$ belongs to $L^{2}\left(0, \infty ; \mathbf{L}^{2}(\Omega)\right)$ and

$$
\|G\|_{L^{2}\left(0, \infty ; \mathbf{L}^{2}(\Omega)\right)} \leq C\left\|\left(v^{0}, \eta_{2}^{0}, g_{p}, F_{f}, F_{\text {div }}, F_{s}\right)\right\| .
$$

Thus, thanks to Theorem 4.1, $(\widehat{v}(t), \widehat{q}(t))$ belongs to $\mathbf{H}_{\delta_{0}}^{2}(\Omega) \times H_{\delta_{0}}^{1}(\Omega)$ and

$$
\|\widehat{v}(t)\|_{\mathbf{H}_{\delta_{0}}^{2}(\Omega)}+\|\widehat{q}(t)\|_{H_{\delta_{0}}^{1}(\Omega)} \leq C\left(\|G(t)\|_{\mathbf{L}^{2}(\Omega)}+\left\|\eta_{2}(t)\right\|_{H_{0}^{\frac{3}{2}}\left(\Gamma_{s}\right)}+\left\|A_{3} \eta_{1}(t)\right\|_{H^{1}(\Omega)}\right) .
$$

We deduce that $(\widehat{v}, \widehat{p})$ belongs to $L^{2}\left(0, \infty ; \mathbf{H}_{\delta_{0}}^{2}(\Omega)\right) \times L^{2}\left(0, \infty ; H_{\delta_{0}}^{1}(\Omega)\right)$ and

$$
\|\widehat{v}\|_{L^{2}\left(0, \infty ; \mathbf{H}_{\delta_{0}}^{2}(\Omega)\right)}+\|\widehat{q}\|_{L^{2}\left(0, \infty ; H_{\delta_{0}}^{1}(\Omega)\right)} \leq C\left\|\left(v^{0}, \eta_{2}^{0}, g_{p}, F_{f}, F_{\text {div }}, F_{s}\right)\right\| .
$$

By combining inequalities (9.4), (9.8) and (9.12), we finally prove that

$$
\left\|\left(v, q, \eta_{1}, \eta_{2}\right)\right\|_{X_{\delta_{0}}} \leq C\left\|\left(v^{0}, \eta_{2}^{0}, g_{p}, F_{f}, F_{\mathrm{div}}, F_{s}\right)\right\| .
$$

10. Stabilization of the nonlinear system. The goal of this section is to prove Theorems 3.3 and 3.2. For that, we are going to show that the feedback law $\mathcal{K}$, stabilizing the linearized system associated to (3.3), is also able to locally stabilize the nonlinear system (3.3). We consider the nonlinear closed-loop system

$$
\begin{aligned}
& \widehat{u}_{t}-\operatorname{div} \sigma(\widehat{u}, \widehat{p})+\left(u_{s} \cdot \nabla\right) \widehat{u}+(\widehat{u} \cdot \nabla) u_{s}-A_{1} \widehat{\eta}_{1}-A_{2} \widehat{\eta}_{2}-\omega \widehat{u}=\mathcal{F}_{f}\left[\widehat{u}, \widehat{p}, \widehat{\eta}_{1}, \widehat{\eta}_{2}\right] \text { in } Q^{\infty}, \\
& \operatorname{div} \widehat{u}=A_{3} \widehat{\eta}_{1}+\operatorname{div} \mathcal{F}_{\operatorname{div}}\left[\widehat{u}, \widehat{\eta}_{1}\right] \text { in } Q^{\infty}, \quad \widehat{u}=\widehat{\eta}_{2} n \text { on } \Sigma_{s}^{\infty}, \quad \widehat{u}=\widehat{g}_{p} \text { on } \Sigma_{i}^{\infty}, \\
& \widehat{u} \cdot n=0 \text { on } \Sigma_{e}^{\infty}, \quad \varepsilon(\widehat{u}) n \cdot \tau=0 \text { on } \Sigma_{e}^{\infty}, \quad \sigma(\widehat{u}, \widehat{p}) n=0 \text { on } \Sigma_{n}^{\infty}, \\
& \widehat{\eta}_{1, t}-\widehat{\eta}_{2}-\omega \widehat{\eta}_{1}=0 \text { on } \Sigma_{s}^{\infty}, \\
& \widehat{\eta}_{2, t}-\beta \Delta_{s} \widehat{\eta}_{1}-\gamma \Delta_{s} \widehat{\eta}_{2}+\alpha \Delta_{s}^{2} \widehat{\eta}_{1}-A_{4} \widehat{\eta}_{1}-\omega \widehat{\eta}_{2}=\gamma_{s} \widehat{p}+\mathcal{F}_{s}\left[\widehat{u}, \widehat{\eta}_{1}\right]+\sum_{i=1}^{n_{c}}\left[\mathcal{K}\left(\widehat{u}, \widehat{\eta}_{1}, \widehat{\eta}_{2}\right)^{T}\right]_{i} w_{i} \text { on } \Sigma_{s}^{\infty}, \\
& \widehat{\eta}_{1}=0 \text { on }(0, \infty) \times \partial \Gamma_{s}, \quad \widehat{\eta}_{1, x}=0 \text { on }(0, \infty) \times \partial \Gamma_{s}, \\
& \widehat{u}(0)=\widehat{u}^{0} \text { in } \Omega, \quad \widehat{\eta}_{1}(0)=0 \text { on } \Gamma_{s}, \quad \widehat{\eta}_{2}(0)=\eta_{2}^{0} \text { on } \Gamma_{s} .
\end{aligned}
$$

Due to the compatibility condition $\left.\widehat{u}^{0}\right|_{\Gamma_{s}}=u^{0}-\left.u_{s}\right|_{\Gamma_{s}}=\eta_{2}^{0} n$, and since $\widehat{\eta}_{1}(0)=0$, the intial condition $\widehat{u}(0)=\widehat{u}^{0}$ is equivalent to $P \widehat{u}(0)=P \widehat{u}^{0}$.

We are going to use the results on the non-homogeneous linear system (9.1) and a fixed point argument to prove that system (10.1) is locally exponentially stable. For that, we introduce the mapping $\mathcal{G}$ from $X_{\delta_{0}}$ into itself, defined by

$$
\mathcal{G}\left(\widetilde{v}, \widetilde{q}, \widetilde{\eta}_{1}, \widetilde{\eta}_{2}\right)=\left(v, q, \eta_{1}, \eta_{2}\right),
$$

where $\left(v, q, \eta_{1}, \eta_{2}\right)$ is the solution of system (9.1) with right hand side

$$
\left(F_{f}, F_{\mathrm{div}}, F_{s}\right)=e^{-\omega t}\left(\mathcal{F}_{f}\left[\widetilde{v}, \widetilde{q}, \widetilde{\eta}_{1}, \widetilde{\eta}_{2}\right], \mathcal{F}_{\mathrm{div}}\left[\widetilde{v}, \widetilde{\eta}_{1}\right], \mathcal{F}_{s}\left[\widetilde{v}, \widetilde{\eta}_{1}\right]\right),
$$


initial data $\left(\widehat{u}^{0}, \eta_{2}^{0}\right)$ in $\mathbf{V}_{\Gamma_{i, e}}^{1}(\Omega) \times H_{0}^{1}\left(\Gamma_{s}\right)$ such that $\widehat{u}^{0}=\eta_{2}^{0} n$ on $\Gamma_{s}$ and boundary perturbation $\widehat{g}_{p}$ in $H_{0}^{1}\left(0, \infty ; \mathbf{H}\left(\Gamma_{i}\right)\right)$. We are going to prove that there exists $R>0$ such that $\mathcal{G}$ is a contraction in

$$
B_{\delta_{0}, R}=\left\{\left(v, q, \eta_{1}, \eta_{2}\right) \in X_{\delta_{0}} \mid\left\|\left(v, q, \eta_{1}, \eta_{2}\right)\right\|_{X_{\delta_{0}}} \leq R \quad \text { and } \quad\left\|e^{-\omega} \eta_{1}\right\|_{L^{\infty}\left(\Sigma_{s}^{\infty}\right)} \leq \eta_{\max }\right\},
$$

where $0<\eta_{\max }<e$.

Lemma 10.1. There exists $C>0$ such that, for all $\eta$ be $H^{4,2}\left(\Sigma_{s}^{\infty}\right)$, we have

$$
\begin{aligned}
& \|\eta\|_{\mathcal{C}_{b}\left([0, \infty) ; \mathcal{C}^{1}\left(\bar{\Gamma}_{s}\right)\right)}+\left\|\eta_{x x}\right\|_{L^{\infty}\left(\Sigma_{s}^{\infty}\right)} \leq C\|\eta\|_{H^{4,2}\left(\Sigma_{s}^{\infty}\right)}, \\
& \left\|\eta_{t}\right\|_{L^{2}\left(0, \infty ; L^{\infty}\left(\Gamma_{s}\right)\right)}+\left\|\eta_{t x}\right\|_{L^{2}\left(0, \infty ; L^{\infty}\left(\Gamma_{s}\right)\right)}+\left\|\eta_{x x x}\right\|_{L^{2}\left(0, \infty ; L^{\infty}\left(\Gamma_{s}\right)\right)} \leq C\|\eta\|_{H^{4,2}\left(\Sigma_{s}^{\infty}\right)}
\end{aligned}
$$

Proof. The results are standard, and their proofs are left to the reader.

Proposition 10.2. There exists $C>0$ such that, for all $\left(v, q, \eta_{1}, \eta_{2}\right) \in B_{\delta_{0}, R}$, we have

$$
\left\|\left(\mathcal{F}_{f}\left[v, p, \eta_{1}, \eta_{2}\right], \mathcal{F}_{\text {div }}\left[v, \eta_{1}\right], \mathcal{F}_{s}\left[v, \eta_{1}\right]\right)\right\|_{Y} \leq C(1+R) R^{2} .
$$

If $\left(v, q, \eta_{1}, \eta_{2}\right)$ and $\left(\widetilde{v}, \widetilde{q}, \widetilde{\eta}_{1}, \widetilde{\eta}_{2}\right)$ belong to $B_{\delta_{0}, R}$, then we have

$$
\begin{aligned}
& \left\|\left(\mathcal{F}_{f}\left[v, p, \eta_{1}, \eta_{2}\right], \mathcal{F}_{\operatorname{div}}\left[v, \eta_{1}\right], \mathcal{F}_{s}\left[v, \eta_{1}\right]\right)-\left(\mathcal{F}_{f}\left[\widetilde{v}, \widetilde{q}, \widetilde{\eta}_{1}, \widetilde{\eta}_{2}\right], \mathcal{F}_{\operatorname{div}}\left[\widetilde{v}, \widetilde{\eta}_{1}\right], \mathcal{F}_{s}\left[\widetilde{v}, \widetilde{\eta}_{1}\right]\right)\right\|_{Y} \\
& \leq C R(1+R)\left\|\left(v, q, \eta_{1}, \eta_{2}\right)-\left(\widetilde{v}, \widetilde{q}, \widetilde{\eta}_{1}, \widetilde{\eta}_{2}\right)\right\|_{X_{\delta_{0}}} .
\end{aligned}
$$

The mapping $\mathcal{G}$ is well-defined. For all $\left(v, q, \eta_{1}, \eta_{2}\right)$ and $\left(\widetilde{v}, \widetilde{q}, \widetilde{\eta}_{1}, \widetilde{\eta}_{2}\right)$ in $B_{\delta_{0}, R}$, we have

$$
\begin{gathered}
\left\|\mathcal{G}\left(v, q, \eta_{1}, \eta_{2}\right)\right\|_{X_{\delta_{0}}} \leq C\left(\left\|\widehat{u}^{0}\right\|_{\mathbf{H}^{1}(\Omega)}+\left\|\eta_{2}^{0}\right\|_{H^{1}\left(\Gamma_{s}\right)}+\left\|\widehat{g}_{p}\right\|_{H_{0}^{1}\left(0, \infty ; \mathbf{H}\left(\Gamma_{i}\right)\right)}+(1+R) R^{2}\right) \\
\left\|\mathcal{G}\left(v, q, \eta_{1}, \eta_{2}\right)-\mathcal{G}\left(\widetilde{v}, \widetilde{q}, \widetilde{\eta}_{1}, \widetilde{\eta}_{2}\right)\right\|_{X_{\delta_{0}}} \leq C R(1+R)\left\|\left(v, q, \eta_{1}, \eta_{2}\right)-\left(\widetilde{v}, \widetilde{q}, \widetilde{\eta}_{1}, \widetilde{\eta}_{2}\right)\right\|_{X_{\delta_{0}}} .
\end{gathered}
$$

Proof. Estimate of $\mathcal{F}_{\text {div }}\left[v, \eta_{1}\right]$. The estimates of $\mathcal{F}_{\text {div }}\left[v, \eta_{1}\right]=-\eta_{1} v_{1} e_{1}+z \eta_{1, x} v_{1} e_{2}$ may be obtained by computing $\mathcal{F}_{\operatorname{div}, x}, \mathcal{F}_{\operatorname{div}, z}, \mathcal{F}_{\mathrm{div}, x x}, \mathcal{F}_{\operatorname{div}, z z}$, and $\mathcal{F}_{\mathrm{div}, t}$, and by using Lemma 10.1 .

Estimate of $\mathcal{F}_{f}\left[v, p, \eta_{1}, \eta_{2}\right]$. To prove that $\mathcal{F}_{f}\left[v, p, \eta_{1}, \eta_{2}\right]$ belongs to $L^{2}\left(Q^{\infty}\right)$, we are going to show that all its terms belong to $L^{2}\left(Q^{\infty}\right)$. By using Lemma 10.1, we can prove that all the nonlinear terms involving either $\eta_{1}$ or $\eta_{2}$ and $v$ belong to $L^{2}\left(Q^{\infty}\right)$. For example, we have

$$
\left\|\eta_{1, x x} v_{z}\right\|_{\mathbf{L}^{2}\left(Q^{\infty}\right)} \leq\left\|\eta_{1, x x}\right\|_{L^{\infty}\left(\Sigma_{s}^{\infty}\right)}\left\|v_{z}\right\|_{\mathbf{L}^{2}\left(Q^{\infty}\right)} \leq C(1+R) R^{2} .
$$

Let us prove that $\eta_{1, x} v_{x z}$ belongs to $L^{2}\left(Q^{\infty}\right)$. We have $\eta_{1, x} \in H^{1}\left(0, \infty ; H_{0}^{1}\left(\Gamma_{s}\right)\right) \hookrightarrow L^{\infty}\left(0, \infty ; H_{0}^{1}\left(\Gamma_{s}\right)\right)$ and $v_{x z} \in L^{2}\left(0, \infty ; \mathbf{L}_{\delta_{0}}^{2}(\Omega)\right)$. Therefore, thanks to Lemma 5.3, we have

$$
\left\|\eta_{1, x} v_{x z}\right\|_{L^{2}\left(Q^{\infty}\right)} \leq C\left\|\eta_{1, x}\right\|_{L^{\infty}\left(0, \infty ; H_{0}^{1}\left(\Gamma_{s}\right)\right)}\left\|v_{x z}\right\|_{L^{2}\left(0, \infty ; \mathbf{L}_{\delta_{0}}^{2}(\Omega)\right)} \leq C(1+R) R^{2} .
$$

Similarly, we can prove that the involving $\eta_{1}$ and second order derivatives of $v$ belong to $L^{2}\left(Q^{\infty}\right)$.

Now, we estimate $(v \cdot \nabla) v$. Since $v \in L^{2}\left(0, \infty ; \mathbf{H}^{\frac{3}{2}+\varepsilon_{0}}(\Omega)\right) \cap H^{1}\left(0, \infty ; \mathbf{L}^{2}(\Omega)\right), v$ belongs to $L^{\infty}(0, \infty$; $\left.\mathbf{H}^{\frac{3}{4}+\frac{\varepsilon_{0}}{2}}(\Omega)\right)$ and $\nabla v$ belongs to $L^{2}\left(0, \infty ; \mathbf{H}^{\frac{1}{2}+\varepsilon_{0}}(\Omega)\right)$. Thanks to [13, Proposition B.1], we have

$$
\|(v \cdot \nabla) v\|_{\mathbf{L}^{2}\left(Q^{\infty}\right)} \leq C\|v\|_{L^{\infty}\left(0, \infty ; \mathbf{H}^{\frac{3}{4}+\frac{\varepsilon_{0}}{2}}(\Omega)\right)}\|\nabla v\|_{L^{2}\left(0, \infty ; \mathbf{H}^{\left.\frac{1}{2}+\varepsilon_{0}(\Omega)\right)}\right.} \leq C(1+R) R^{2} .
$$

Thus, $\mathcal{F}_{f}\left[v, p, \eta_{1}, \eta_{2}\right]$ belongs to $\mathbf{L}^{2}\left(Q^{\infty}\right)$ and $\left\|\mathcal{F}_{f}\left[v, p, \eta_{1}, \eta_{2}\right]\right\|_{\mathbf{L}^{2}\left(Q^{\infty}\right)} \leq C(1+R) R^{2}$.

Estimate of $\mathcal{F}_{s}\left[v, \eta_{1}\right]$. We can easily prove that $\mathcal{F}_{s}\left[v, \eta_{1}\right]$ belongs to $L^{2}\left(\Sigma_{s}^{\infty}\right)$ when $v$ belongs to $H_{\delta}^{2,1}\left(Q_{\infty}\right)$ and $\eta_{1}$ belongs to $H^{4,2}\left(\Sigma_{s}^{\infty}\right)$.

The Lipschitz estimates in (10.3) can be obtained with the same arguments as above. They follow from the fact that all the terms involved in the calculations are at least quadratic with respect to $\left(v, q, \eta_{1}, \eta_{2}\right)$ and $\left(\widetilde{v}, \widetilde{q}, \widetilde{\eta}_{1}, \widetilde{\eta}_{2}\right)$.

The estimates in (10.4) follows from estimates (10.2) and (10.3), and from Theorem 9.2. 
THEOREM 10.3. Let $\eta_{\max }$ be in $(0, e)$ and $\omega$ be positive. There exists $r>0$ such that, for all $\left(\widehat{u}^{0}, \eta_{2}^{0}\right) \in \mathbf{V}_{\Gamma_{i, e}}^{1}(\Omega) \times H_{0}^{1}\left(\Gamma_{s}\right)$ and $\widehat{g}_{p} \in H_{0}^{1}\left(0, \infty ; \mathbf{H}\left(\Gamma_{i}\right)\right)$ satisfying $\widehat{u}^{0}=\eta_{2}^{0} n$ on $\Gamma_{s}, \widehat{g}_{p} \in H_{0}^{1}\left(0, \infty ; \mathbf{H}\left(\Gamma_{i}\right)\right)$ and

$$
\left\|\widehat{u}^{0}\right\|_{\mathbf{H}^{1}(\Omega)}+\left\|\eta_{2}^{0}\right\|_{H^{1}\left(\Gamma_{s}\right)}+\left\|\widehat{g}_{p}\right\|_{H_{0}^{1}\left(0, \infty ; \mathbf{H}\left(\Gamma_{i}\right)\right)} \leq r
$$

system (10.1) admits a solution $\left(\widehat{u}, \widehat{p}, \widehat{\eta}_{1}, \widehat{\eta}_{2}\right) \in X_{\delta_{0}}$ satisfying the estimates

$$
\left\|e^{-\omega t} \widehat{\eta}_{1}(t)\right\|_{L^{\infty}\left(\Gamma_{s}\right)} \leq \eta_{\max } \quad \text { and } \quad\left\|\left(\widehat{u}(t), \widehat{\eta}_{1}(t), \widehat{\eta}_{2}(t)\right)\right\|_{\mathbf{H}^{\frac{3}{4}}+\frac{\varepsilon_{0}}{2}(\Omega) \times H^{3}\left(\Gamma_{s}\right) \times H^{1}\left(\Gamma_{s}\right)} \leq C, \quad \forall t>0 .
$$

Proof. We choose $R_{0}>0$ small enough such that

$$
\frac{R_{0}}{2}+C\left(1+R_{0}\right) R_{0}^{2}<R_{0}, \quad C R_{0}\left(1+R_{0}\right)<1, \quad \text { and } \quad C R_{0}<\eta_{\max }
$$

and we set $r=2 C R_{0}$, where $C$ is the constant appearing in (10.4). Due to (10.4), with such a choice, $\mathcal{G}$ is a contraction in $B_{\delta_{0}, R_{0}}$, and system (10.1) admits a unique solution $\left(\widehat{u}, \widehat{q}, \widehat{\eta}_{1}, \widehat{\eta}_{2}\right) \in B_{\delta_{0}, R_{0}}$ satisfying the estimates

$$
\left\|e^{-\omega \cdot} \widehat{\eta}_{1}\right\|_{L^{\infty}\left(\Sigma_{s}^{\infty}\right)} \leq \eta_{\max } \quad \text { and } \quad\left\|\left(\widehat{u}, \widehat{p}, \widehat{\eta}_{1}, \widehat{\eta}_{2}\right)\right\|_{X_{\delta_{0}}} \leq R_{0} .
$$

From [18, Theorem 2.1] and Lemma 4.3, it follows that

$$
\|\widehat{u}(t)\|_{\mathbf{H}^{\frac{3}{4}}+\frac{\varepsilon_{0}}{2}(\Omega)}+\left\|\widehat{\eta}_{1}(t)\right\|_{H^{3}\left(\Gamma_{s}\right)}+\left\|\widehat{\eta}_{2}(t)\right\|_{H^{1}\left(\Gamma_{s}\right)} \leq C R_{0} .
$$

Proof of Theorem 3.2. Thanks to Theorem 10.3, we have to check that, for all $\eta \in H^{4,2}\left(\Sigma_{s}^{\infty}\right)$ such that $\|\eta\|_{L^{\infty}\left(\Sigma_{s}^{\infty}\right)} \leq \eta_{\max }$, and all $t \in(0, \infty), \mathcal{T}_{\eta(t)}$ is a $\mathcal{C}^{1}$-diffeomorphism from $\Omega_{\eta(t)}$ into $\Omega$. For that, it is enough to use the continuous embedding $H^{4,2}\left(\Sigma_{s}^{\infty}\right) \hookrightarrow \mathcal{C}_{b}\left([0, \infty) ; \mathcal{C}^{1}\left(\Gamma_{s}\right)\right)$ (see Lemma 10.1) and the estimate $\|\eta\|_{L^{\infty}\left(\Sigma_{s}^{\infty}\right)} \leq \eta_{\max }$.

Acknowledgments. The authors are partially supported by the Thematic Network 'Aeronautic and Space' via the project CARPE (Contrôle Actif et Robuste de Plaque Epaisse). The last two authors are partially supported by the ANR-Project IFSMACS (ANR 15-CE40.0010).

Appendix A. Nonlinear terms. The nonlinear terms $\mathcal{F}_{f}$ and $\mathcal{F}_{s}$ in system (3.3) are defined by

$$
\begin{aligned}
\mathcal{F}_{f}[\widehat{u}, \widehat{p}, & \left.\widehat{\eta}_{1}, \widehat{\eta}_{2}\right]=\frac{(\ell-s(z) z) \widehat{\eta}_{2} \widehat{u}_{z}}{\ell-e}-\frac{\nu(\ell-s(z) z) \widehat{\eta}_{1, x x} \widehat{u}_{z}}{\ell-e}-\frac{2 \nu(\ell-s(z) z) \widehat{\eta}_{1, x} \widehat{u}_{x z}}{\ell-e} \\
& +\frac{2 \nu s(z) \widehat{\eta}_{1} \widehat{u}_{z z}}{\ell-e}-\nu \nabla \operatorname{div} \mathcal{F}_{\operatorname{div}}\left[\widehat{u}, \widehat{\eta}_{1}\right]+\frac{(\ell-s(z) z) \widehat{\eta}_{1, x} \widehat{p}_{z} e_{1}}{\ell-e}-\frac{s(z) \widehat{\eta}_{1} \widehat{p}_{z} e_{2}}{\ell-e} \\
& +\frac{(\ell-s(z) z) e^{-\omega t} \widehat{\eta}_{1, x} \widehat{u}_{1} \widehat{u}_{z}}{\ell-e}+\frac{(\ell-s(z) z) \widehat{\eta}_{1, x} \widehat{u}_{1} u_{s, z}}{\ell-e}+\frac{(\ell-s(z) z) \widehat{\eta}_{1, x} u_{s, 1} \widehat{u}_{z}}{\ell-e} \\
& -\frac{e^{-\omega t} s(z) \widehat{\eta}_{1} \widehat{u}_{2} \widehat{u}_{z}}{\ell-e}-\frac{s(z) \widehat{\eta}_{1} \widehat{u}_{2} u_{s, z}}{\ell-e}-\frac{s(z) \widehat{\eta}_{1} u_{s, 2} \widehat{u}_{z}}{\ell-e}+\frac{(\ell-s(z) z) s(z) \widehat{\eta}_{1} \widehat{\eta}_{2}\left(e^{-\omega t} \widehat{u}_{z}+u_{s, z}\right)}{(\ell-e)\left(\ell-e-s(z) e^{-\omega t} \widehat{\eta}_{1}\right)} \\
& +\frac{\nu(\ell-s(z) z) s(z) \widehat{\eta}_{1} \widehat{\eta}_{1, x x}\left(e^{-\omega t} \widehat{u}_{z}+u_{s, z}\right)}{(\ell-e)\left(\ell-e-s(z) e^{-\omega t} \widehat{\eta}_{1}\right)}+\frac{2 \nu(\ell-s(z) z) \widehat{\eta}_{1, x}^{2}\left(e^{-\omega t} \widehat{u}_{z}+u_{s, z}\right)}{\left(\ell-e-s(z) e^{-\omega t} \widehat{\eta}_{1}\right)^{2}} \\
& -\frac{2 \nu(\ell-s(z) z) s(z) \widehat{\eta}_{1} \widehat{\eta}_{1, x}\left(e^{-\omega t} \widehat{u}_{x z}+u_{s, x z}\right)}{(\ell-e)\left(\ell-e-s(z) e^{-\omega t} \widehat{\eta}_{1}\right)}+\frac{\nu\left((\ell-s(z) z)^{2} \widehat{\eta}_{1, x}^{2}-\widehat{\eta}_{1}^{2}\right)\left(e^{-\omega t} \widehat{u}_{z z}+u_{s, z z}\right)}{\left(\ell-e-s(z) e^{-\omega t} \widehat{\eta}_{1}\right)^{2}} \\
& +\frac{2 \nu\left(\ell-e-s(z) e^{-\omega t} \widehat{\eta}_{1}\right) \widehat{\eta}_{1}^{2}\left(e^{-\omega t} \widehat{u}_{z z}+u_{s, z z}\right)}{(\ell-e)\left(\ell-e-s(z) e^{-\omega t} \widehat{\eta}_{1}\right)^{2}}+\frac{(\ell-s(z) z) s(z) \widehat{\eta}_{1} \widehat{\eta}_{1, x}\left(e^{-\omega t} \widehat{p}_{z}+p_{s, z}\right) e_{1}}{(\ell-e)\left(\ell-e-s(z) e^{-\omega t} \widehat{\eta}_{1}\right)} \\
& -\frac{\widehat{\eta}_{1}^{2}\left(e^{-\omega t} \widehat{p}_{z}+p_{s, z}\right) e_{2}}{(\ell-e)\left(\ell-e-s(z) e^{-\omega t} \widehat{\eta}_{1}\right)}+\frac{(\ell-s(z) z) s(z) \widehat{\eta}_{1} \widehat{\eta}_{1, x}\left(e^{-\omega t} \widehat{u}_{1}+u_{s, 1}\right)\left(e^{-\omega t} \widehat{u}_{z}+u_{s, z}\right)}{(\ell-e)\left(\ell-e-s(z) e^{-\omega t} \widehat{\eta}_{1}\right)} \\
& -\frac{\widehat{\eta}_{1}^{2}\left(e^{-\omega t} \widehat{u}_{2}+u_{s, 2}\right)\left(e^{-\omega t} \widehat{u}_{z}+u_{s, z}\right)}{(\ell-e)\left(\ell-e-s(z) e^{-\omega t} \widehat{\eta}_{1}\right)}-e^{-\omega t}(\widehat{u} \cdot \nabla) \widehat{u},
\end{aligned}
$$




$$
\begin{aligned}
\mathcal{F}_{s}\left[\widehat{u}, \widehat{\eta}_{1}\right] & =\nu\left(\widehat{\eta}_{1, x} \widehat{u}_{1, z}+\widehat{\eta}_{1, x} \widehat{u}_{2, x}+\frac{2 s(z) \widehat{\eta}_{1} \widehat{u}_{2, z}}{\ell-e}\right)-\frac{\nu \widehat{\eta}_{1} \widehat{\eta}_{1, x}\left(e^{-\omega t} \widehat{u}_{1, z}+u_{s, 1, z}\right)}{\ell-e-s(z) e^{-\omega t} \widehat{\eta}_{1}} \\
& -\frac{2 \nu \widehat{\eta}_{1}^{2}\left(e^{-\omega t} \widehat{u}_{2, z}+u_{s, 2, z}\right)}{(\ell-e)\left(\ell-e-s(z) e^{-\omega t} \widehat{\eta}_{1}\right)}-\frac{\nu(\ell-e) \eta_{1, x}^{2}\left(e^{-\omega t} \widehat{u}_{2, z}+u_{s, 2, z}\right)}{\ell-e-s(z) e^{-\omega t} \widehat{\eta}_{1}}-2 \nu \gamma_{s}\left(\operatorname{div} \mathcal{F}_{\operatorname{div}}\left[\widehat{u}, \widehat{\eta}_{1}\right]\right) .
\end{aligned}
$$

In the definition of the nonlinear terms, as indicated in Remark 3.1, the extension operator $\mathcal{E}$ is implicitly present in front of all the terms involving $\widehat{\eta}_{1}$ or $\widehat{\eta}_{2}$.

\section{REFERENCES}

[1] M. BADRA AND T. TAKAhashi, Feedback boundary stabilization of 2D fluid-structure interaction systems, Discrete Contin. Dyn. Syst., 37 (2017), pp. 2315-2373.

[2] H. BEIRÃo DA VEIGA, On the existence of strong solutions to a coupled fluid-structure evolution problem, J. Math. Fluid Mech., 6 (2004), pp. 21-52.

[3] A. Bensoussan, G. Da Prato, M. C. Delfour, and S. K. Mitter, Representation and control of infinitedimensional systems. Vol. I, Systems \& Control: Foundations \& Applications, Birkhäuser Boston, Inc., Boston, MA, 1992.

[4] - Representation and control of infinite-dimensional systems. Vol. II, Systems \& Control: Foundations \& Applications, Birkhäuser Boston, Inc., Boston, MA, 1993.

[5] P. Causin, J. F. Gerbeau, and F. Nobile, Added-mass effect in the design of partitioned algorithms for fluidstructure problems, Comput. Methods Appl. Mech. Engrg., 194 (2005), pp. 4506-4527.

[6] A. Chambolle, B. Desjardins, M. J. Esteban, and C. Grandmont, Existence of weak solutions for the unsteady interaction of a viscous fluid with an elastic plate, J. Math. Fluid Mech., 7 (2005), pp. 368-404.

[7] S. P. Chen and R. Triggiani, Proof of extensions of two conjectures on structural damping for elastic systems, Pacific J. Math., 136 (1989), pp. 15-55.

[8] M. FourniÉ, M. Ndiaye, and J.-P. Raymond, Numerical stabilization of a fluid-structure interaction system, submitted, 2018

[9] A. V. Fursikov, Stabilizability of a quasilinear parabolic equation by means of boundary feedback control, Mat. Sb., 192 (2001), pp. 115-160.

[10] C. Grandmont, Existence of weak solutions for the unsteady interaction of a viscous fluid with an elastic plate, SIAM J. Math. Anal., 40 (2008), pp. 716-737.

[11] C. Grandmont And M. Hillairet, Existence of global strong solutions to a beam-fluid interaction system, Arch. Ration. Mech. Anal., 220 (2016), pp. 1283-1333.

[12] P. Grisvard, Singularities in boundary value problems, Vol. 22 of Recherches en Mathématiques Appliquées, Masson, Paris; Springer-Verlag, Berlin, 1992.

[13] G. GRubB And V. A. Solonnikov, Boundary value problems for the nonstationary Navier-Stokes equations treated by pseudo-differential methods, Math. Scand., 69 (1991), pp. 217-290.

[14] S. Kesavan And J.-P. Raymond, On a degenerate Riccati equation, Control Cybernet., 38 (2009), pp. $1393-1410$.

[15] V. A. Kozlov, V. G. Maz'ya, And J. Rossmann, Spectral problems associated with corner singularities of solutions to elliptic equations, Vol. 85 of Mathematical Surveys and Monographs, American Mathematical Society, Providence, RI, 2001.

[16] J. LEQUEURRE, Existence of strong solutions to a fluid-structure system, SIAM J. Math. Anal., 43 (2011), pp. 389-410.

[17] J.-L. Lions and E. Magenes, Problèmes aux limites non homogènes et applications. Vol. 1, Travaux et Recherches Mathématiques, No. 17, Dunod, Paris, 1968.

[18] _ Non-homogeneous boundary value problems and applications. Vol. II, Springer-Verlag, New York-Heidelberg, 1972. Translated from the French by P. Kenneth, Die Grundlehren der mathematischen Wissenschaften, Band 182.

[19] J. Rossmann, On two classes of weighted Sobolev-Slobodetski spaces in a dihedral angle, Partial differential equations, Part 1, 2 (Warsaw, 1990), 399-424, Banach Center Publ., 27, Warsaw, 1992.

[20] V. G. Maz'ya And J. Rossmann, Elliptic equations in polyhedral domains, Vol. 162 of Mathematical Surveys and Monographs, American Mathematical Society, Providence, RI, 2010.

[21] P. A. NGuYen And J.-P. Raymond, Boundary stabilization of the Navier-Stokes equations in the case of mixed boundary conditions, SIAM J. Control Optim., 53 (2015), pp. 3006-3039.

[22] A. Osses AND J.-P. Puel, Approximate controllability for a linear model of fluid structure interaction, ESAIM Control Optim. Calc. Var., 4 (1999), pp. 497-513.

[23] — Unique continuation property near a corner and its fluid-structure controllability consequences, ESAIM Control Optim. Calc. Var., 15 (2009), pp. 279-294.

[24] J.-P. RaYmond, Feedback stabilization of a fluid-structure model, SIAM J. Control Optim., 48 (2010), pp. $5398-5443$.

[25] J.-P. Raymond and L. Thevenet, Boundary feedback stabilization of the two dimensional Navier-Stokes equations with finite dimensional controllers, Discrete Contin. Dyn. Syst., 27 (2010), pp. 1159-1187.

[26] M. Tucsnak And G. Weiss, Observation and control for operator semigroups, Birkhäuser Advanced Texts, Birkhäuser Verlag, Basel, 2009. 